\title{
The rates of type la supernovae
}

\section{Analytical formulations ${ }^{\star}$}

\author{
L. Greggio ${ }^{1}$
}

INAF - Osservatorio Astronomico di Padova, vicolo dell'Osservatorio 5, 35122 Padova, Italy

e-mail: greggio@pd.astro.it

Received 23 February 2005 / Accepted 15 April 2005

\section{ABSTRACT}

The aim of this paper is to provide a handy tool to compute the impact of type Ia SN (SNIa) events on the evolution of stellar systems. An effective formalism to couple the rate of SNIa explosions from a single burst of star formation and the star formation history is presented, which rests upon the definition of the realization probability of the SNIa event $\left(A_{\mathrm{Ia}}\right)$ and the distribution function of the delay times $\left(f_{\mathrm{Ia}}(\tau)\right)$. It is shown that the current SNIa rate in late type galaxies constrains $A_{\text {Ia }}$ to be on the order of $10^{-3}$ (i.e. 1 SNIa every $1000 M_{\odot}$ of gas turned into stars), while the comparison of the current rates in early and late type galaxies implies that $f_{\text {Ia }}$ ought to be more populated at short delays. The paper presents analytical formulations for the description of the $f_{\text {Ia }}$ function for the most popular models of SNIa progenitors, namely Single Degenerates (Chandrasekhar and Sub-Chandrasekhar exploders), and Double Degenerates. These formulations follow entirely from general considerations on the evolutionary behavior of stars in binary systems, modulo a schematization of the outcome of the phases of mass exchange, and compare well with the results of population synthesis codes, for the same choice of parameters. The derivation presented here offers an immediate astrophysical interpretation of the shape of the $f_{\mathrm{Ia}}$ functions, and have a built in parametrization of the key properties of the alternative candidates. The important parameters appear to be the minimum and maximum masses of the components of the binary systems giving rise to a SNIa explosions, the distribution of the primary mass and of the mass ratios in these systems, the distribution of the separations of the DD systems at their birth. The various models for the progenitors correspond to markedly different impact on the large scales; correspondingly, the model for the progenitor can be constrained by examining the relevant observations. Among these, the paper concentrates on the trend of the current SNIa rate with parent galaxy type. The recent data by Mannucci et al. (2005, A\&A, 433, 807) favor the DD channel over the SD one, which tends to predict a too steep distribution function of the delay times. The SD scenario can be reconciled with the observations only if the distribution of the mass ratios in the primordial binaries is flat and the accretion efficiency onto the WD is close to $100 \%$. The various models are characterized by different timescales for the Fe release from a single burst stellar population. In particular the delay time within which half of the SNIa events from such a population have occurred, ranges between 0.3 and 3 Gyr, for a wide variety of hypothesis on the progenitors.

Key words. stars: binaries: close - stars: supernovae: general - galaxies: evolution - galaxies: intergalactic medium stars: white dwarfs

\section{Introduction}

The evolution of the rate of type Ia Supernovae (SNIa) with time is a fundamental ingredient for the study of a variety of astrophysical issues, ranging from the chemical evolution of stellar systems, to the interpretation of the SNIa rates as a function of redshift.

Indeed, while type II Supernovae (SNII) precursors are short lived, massive stars, so that their rate evolves (almost) in pace with the rate of star formation, SNIa come from binary systems with a wide range of lifetimes, as indicated by their occurrence in both late and early type galaxies. Thus,

* Appendix A is only available in electronic form at http://www . edpsciences.org the SNIa products are released to the interstellar medium over longer timescales, compared to SNII products. Since the $\alpha$ elements are mostly produced by SNII, while SNIa are important contributors of iron, the shorter the formation timescale of a stellar system is, the higher the $\alpha$ to Fe abundance ratio recorded in its stars. This argument is at the basis of the evaluation of the formation timescales of stellar systems from the $\alpha$ to $\mathrm{Fe}$ abundance ratios, and has been used to estimate the formation timescale of the halo of our galaxy from individual stellar abundances (e.g. Matteucci \& Greggio 1986), as well as to infer short formation timescales for Es from the Magnesium and Iron indices in their spectra (e.g. Matteucci 1994; Greggio 1997; Thomas et al. 2005). SNIas are also thought to produce a major fraction of the iron in the intracluster medium in galaxy 
clusters (e.g. Matteucci \& Vettolani 1988; Renzini 1997); the temporal behavior of the SNIa rate will then impact on the iron abundance of the intracluster gas at high redshift.

Type Ia SNe provide the heating mechanism of the mass lost by stars in Ellipticals, an therefore determine the dynamical evolution of the gas in these galaxies. Following Renzini (1996), this evolution depends on the balance between the rate of mass return, and the secular evolution of the SNIa rate: if the latter decreases faster than the former, the early stages are characterized by supersonic winds, which then turn to subsonic outflows, and eventually to inflows (Ciotti et al. 1991). The reverse sequence applies instead in the case of a mild secular evolution of the SNIa rate, with early inflows eventually turning into winds, such as in the models of Loewenstein \& Mathews (1987).

Finally, cosmological applications of the SNIa rate include the possibility of deriving clues on the SNIa progenitors, and/or constraining the cosmic star formation history from the evolution of the SNIa rate with redshift (Madau et al. 1998; Strolger et al. 2004). Understanding the evolutionary path which leads to a type Ia explosion is of great importance to assess the use of SNIa as distance indicators, and to derive the cosmological parameters (Riess et al. 1998; Perlmutter et al. 1999).

To address these issues and quantitatively interpret the related observations we need a suitable description of the evolution of the SNIa rate from a single burst stellar population. From a theoretical point of view, this rate is difficult to derive, first because the nature of the progenitors of SNIa events is still an open question, and, second, because any theoretical rendition is highly model dependent. While in the literature there is a general consensus that SNIa originate form the thermonuclear explosion of carbon and Oxygen (CO) White Dwarfs (WD), various evolutionary paths may lead to such event. Common to all the models is the first part of the evolution, dealing with a close binary system with a primary component less massive than $\sim 8 M_{\odot}$, so that it evolves into a CO WD. When the secondary component expands and fills its Roche Lobe, the primary may or may not accrete the matter shed by its companion. If the accretion rate is approximately $\sim 10^{-7} M_{\odot} / \mathrm{yr}$ (Nomoto 1982), the accreted matter burns on top of the WD, the object remains confined within its Roche Lobe and grows in mass (Whelan \& Iben 1973). However, Iben \& Tutukov (1984) pointed out that in most cases the secondary expands at such a high rate that the accretion rate exceeds the mentioned limit, implying that a common envelope (CE) forms around the two stars. Orbiting inside the CE the two cores spiral in, and orbital energy is transferred to the envelope which is eventually lost. The system emerges form the CE phase as a close double WD, which will merge due to the emission of gravitational wave radiation. Another interesting possibility is that, when subject to a large accretion rate, the WD develops a strong radiative wind, to the effect of stabilizing the mass transfer, thus allowing the WD to grow in mass, and eventually explode (Hachisu et al. 1996). Within all scenarios explosion occurs either when the CO WD reaches the Chandrasekhar mass and carbon deflagrates at the center (Chandra exploders), or when a massive enough helium layer is accumulated on top of the $\mathrm{CO} \mathrm{WD}$, so that helium detonates, inducing off center carbon detonation (e.g. Woosley \& Weaver 1994) before the Chandrasekhar mass is reached (Sub-Chandra exploders).

Different arguments can be found in favor or against both scenarios (e.g. Livio 2001), generally referred to as Single Degenerate (SD) and Double Degenerate (DD), depending on whether the SNIa precursor is a system with one or two WDs. Briefly, the SD model is supported by the observational detection of several classes of objects that can be considered as potential SNIa precursors of the SD variety, i.e. Cataclysmic Variables, Symbiotic Stars, and Supersoft X-Ray Sources (Munari \& Renzini 1992; Kenyon et al. 1993; Van den Heuvel et al. 1992; Rappaport, Di Stefano \& Smith 1994). Additional support to the Cataclysmic Binaries channel came recently from the detection of a candidate companion to Tycho's supernova (Ruiz-Lapuente et al. 2004). On the other hand, the fine tuning of the mass accretion rate limits considerably the volume in the parameter space for a successful SNIa explosion in the SD model. As a consequence, it seems likely that only a small fraction of events can be realized through this channels in our galaxy (Fedorova et al. 2004; Han \& Podsiadlowski 2004, but see Hachisu et al. 1999, for a different point of view).

Several attempts have been made to establish the binary frequency among White Dwarfs, and to determine the distribution of total masses and periods of the binary systems, in order to assess the likelihood of the DD channel as SNIa progenitor (Robinson \& Shafter 1987; Bragaglia et al. 1990; Foss et al. 1991; Saffer et al. 1998; Maxted \& Marsh 1999). To date, the most comprehensive effort to find SNIa precursors among DD systems is the SPY project (Napiwotzki et al. 2001), whose results have been recently summarized in Napiwotzki et al. (2004): many close DD systems have been found, with one very good candidate SNIa precursor, with a total mass exceeding the Chandrasekhar limit and expected to merge within a Hubble time. In addition, a few other systems come close to qualify as SNIa precursors. In general, it seems that the masses and period distributions of the binary WDs confirm the prediction of the population synthesis models; according to these models the DD evolutionary channel is able to provide enough merging events to match the current SNIa rate measured in our galaxy, which is similar to the typical SNIa rate in Spirals. On the other hand, theoretical calculations show that the merging of two massive WDs may lead to accretion induced collapse, rather than to SNIa explosion (Saio \& Nomoto 1998), so that the ultimate fate of these candidates may be a neutron star.

The various models for the progenitors, SD or DD, undergoing Chandra or Sub-Chandra explosions, correspond to rather different temporal behavior of the SNIa rate (see e.g. Fig. 2 in Yungelson \& Livio 2000, hereafter YL). In the current literature there are several examples of theoretical computations of the SNIa rate performed with population synthesis codes: starting from a primordial distributions of binary masses, mass ratios and separations, the computations follow the evolution of the stellar systems under some prescriptions for the mass exchange between the binary components, to determine the final outcome (Tutukov \& Yungelson 1994; Yungelson et al. 1994; Ruiz-Lapuente et al. 1995; Han et al. 1995; Nelemans et al. 2001; De Donder \& Vanbeveren 2003). The results of these simulations depend on a variety of input 
parameters and assumptions, whose role is difficult to gauge, so that they are not suited to easily explore the parameter space for the SNIa progenitors' models. In addition, the population synthesis codes yield numerical outputs, which are difficult to incorporate in codes which follow the evolution of galaxies or of galaxy clusters. Indeed, most of the astrophysical applications of the SNIa rates in the literature are based either on the analytical formulation by Greggio \& Renzini (1983), or on the parametrization proposed by Madau et al. (1998). However, the former is derived only in the framework of the SD model; the latter is a convenient mathematical expression, but it is only marginally related to the physics of stellar evolution.

In this paper I provide relatively simple analytical formulation for the SNIa rate, which allows us to identify the most critical parameters and should help restrict the choice among the candidate precursors. Both the SD (Chandra and Sub-Chandra) and the DD (only Chandra) models are considered, so as to provide a handy way for investigating on the impact of the different SNIa models on astrophysical issues for which the SNIa rate is important. Section 2 presents a coherent formalism to couple a particular SNIa model with the star formation history of a system. The analytic expressions for the SNIa rate for the SD and DD models are derived in Sects. 3 and 4 respectively. Readers mostly interested in the main results may skip these sections, since a general description of the analytic $f_{\mathrm{Ia}}$ function appears at the beginning of Sect. 5, where they are compared to the predictions of population synthesis codes. In addition, Sect. 5 presents an attempt to constrain the SNIa progenitors from the systematic trend of the SNIa rates with galaxy type. Finally, some concluding remarks appear in Sect. 6. The mathematics used to derive the analytic relation for the DD model is (mostly) described in the Appendix, for an easier readability of the text.

\section{Formalism}

A convenient formulation of the SNIa rate to follow the evolution of stellar systems rests upon the definition of the distribution function of the delay times, i.e. the time elapsed between the birth of a SNIa progenitor and its explosion. I indicate this function with $f_{\mathrm{Ia}}(\tau)$, defined in the range $\left(\tau_{\mathrm{i}}, \tau_{\mathrm{x}}\right)$, respectively the minimum and maximum possible delay times, and consider $f_{\mathrm{Ia}}(\tau)$ normalized to $1: \int_{\tau_{\mathrm{i}}}^{\tau_{\mathrm{x}}} f_{\mathrm{Ia}}(\tau) \cdot \mathrm{d} \tau=1$. The minimum delay time $\tau_{\mathrm{i}}$ is the minimum evolutionary lifetime of the SNIa precursors: for the SD model this is the nuclear lifetime of the most massive stars which produce a WD, that is an $\sim 8 M_{\odot}$ star, which evolves off the MS in $\sim 0.04$ Gyr. For the DD model, $\tau_{\mathrm{i}}$ could be appreciably larger than this because of the additional gravitational delay, i.e. the time taken by the DD system to merge due to the gravitational wave radiation. The maximum delay time $\tau_{\mathrm{x}}$ is quite sensitive to the model for the SNIa precursor, as will be seen later. At this point I just notice that, if elliptical galaxies formed the bulk of their stars in a short initial burst, the maximum delay time of their inhabiting SNIa precursors must be on the order of a Hubble time, or more.

At a given epoch $t$, the contribution to the SNIa rate from progenitors with delay times in the range $(\tau, \tau+\mathrm{d} \tau)$ is

$\mathrm{d} \dot{n}_{\mathrm{Ia}}=\dot{n}_{\star}(t-\tau) \times A_{\mathrm{Ia}}(t-\tau) \times f_{\mathrm{Ia}}(\tau) \mathrm{d} \tau$ where $\dot{n}_{\star}(t-\tau)$ is the birth rate at epoch $(t-\tau)$, and $A_{\mathrm{Ia}}$ is the realization probability of the SNIa scenario from the stellar generation born at $(t-\tau)^{1}$. In Eq. (1) I have considered the possibility of variations of $A_{\text {Ia }}$ during the galaxy evolution. For example, one could expect larger realization probabilities of the SNIa channel at higher metallicities because, during their evolution, stars expand to larger radii (Greggio \& Renzini 1990), hence have a better chance to fill their Roche Lobe. In addition, at high metallicity the accretion onto the pre-supernova WD could be more efficient (Hachisu et al. 1996), also implying larger values for $A_{\text {Ia }}$.

Following Tinsley (1980) notation:

$\dot{n}_{\star}(t-\tau)=\psi(t-\tau) \times \int_{m_{i}}^{m_{s}} \phi(m) \mathrm{d} m$

where $\phi(m)$ is the initial mass function (IMF) by number, and $m_{i}$ and $m_{s}$ are the lower and upper mass limits. As usual, I adopt a power law IMF, with total mass normalized to 1, so that the star formation rate (SFR) $\psi$ is the mass that goes into stars per unit time. It follows:

$\dot{n_{\star}}(t-\tau)=\psi(t-\tau) \times k_{\alpha}$

where $k_{\alpha}$, which is the number of stars per unit mass in one stellar generation, depends on the IMF. For example, for a mass distribution $\phi(m) \propto m^{-\alpha}$ ranging from 0.1 to $120 M_{\odot}, k_{\alpha}$ is equal to 2.83 and 1.55 , respectively for Salpeter $(\alpha=2.35)$ and Kroupa (2001) $\left(\alpha=2.3\right.$ in $m \geq 0.5 M_{\odot}$ and $\alpha=1.3$ in $\left.m \leq 0.5 M_{\odot}\right)$ IMFs.

By substituting Eqs. (3) into (1), and summing over all the contributions from the past stellar generations, the SNIa rate at epoch $t$ is:

$\dot{n}_{\mathrm{Ia}}(t)=k_{\alpha} \times \int_{\tau_{\mathrm{i}}}^{\min \left(t, \tau_{\mathrm{x}}\right)} \psi(t-\tau) A_{\mathrm{Ia}}(t-\tau) f_{\mathrm{Ia}}(\tau) \mathrm{d} \tau$

This equation allows one to insert consistently the SNIa events in codes which describe the evolution of galaxies, once the distribution of the delay times $f_{\text {Ia }}$ and the fraction $A_{\text {Ia }}$ are specified. Notice that these are the only results of the modeling of binary populations of SNIa precursors which impact on the evolution of stellar systems. In other words, for the astrophysical applications, the particular prescriptions used in the population synthesis codes are of limited interest, while most important is the distribution function of the delay times and the total realization probability of the SNIa scenario out of one stellar generation.

Equation (4) can be easily specified for a single generation of stars by considering a star formation episode started at $t=0$ and proceeded at a constant rate $\psi_{\mathrm{B}}$ for a very short time $\left(\Delta t_{\mathrm{B}}\right)$. In this case, the integrand function is non zero only in a narrow age range around $\tau=t$ so that:

$$
\begin{aligned}
\dot{n}_{\mathrm{Ia}}(t) & =k_{\alpha} \cdot \psi_{\mathrm{B}} \cdot \Delta t_{\mathrm{B}} \cdot A_{\mathrm{Ia}}(t=0) \cdot f_{\mathrm{Ia}}(\tau=t) \\
& =k_{\alpha} \cdot \mathcal{M}_{\mathrm{B}} \cdot A_{\mathrm{Ia}} \cdot f_{\mathrm{Ia}}(t)
\end{aligned}
$$

where $\mathcal{M}_{\mathrm{B}}$ is the mass that went into stars in the burst. Thus the SNIa rate following an instantaneous burst of star formation is proportional to the distribution function of the delay

\footnotetext{
${ }^{1}$ If $N_{\star}$ is the number of stars born at epoch $(t-\tau), A_{\mathrm{Ia}} \times N_{\star}$ is the number of SNIa events produced by this stellar generation ever.
} 
times through a factor which is the product of the total stellar mass formed in the burst, times the realization probability of the SNIa scenario, times the number of stars per unit mass, characteristic of the IMF.

\subsection{Observational constraints}

Equation (4) shows that the SNIa rate results from the convolution of the distribution function of the delay times and the SF history: in order to derive clues on the former from the observed rates I consider a family of models, characterized by a constant SFR $\left(\psi_{0}\right)$ starting at $t=0$, and with variable durations $(\Delta t)$. I also assume that early (late) type galaxies are represented by models with short (long) $\Delta t$. Although the SF history in real galaxies is much more complex than that, this schematic description leads to interesting relations between the observed SNIa rates and the key quantities $A_{\text {Ia }}$ and $f_{\text {Ia }}$, which will not be far from those that one can obtain with a thorough modeling of the galaxy evolution.

For this family of models, the minimum delay time which contributes to the rate at epoch $t$ is either $\tau_{\mathrm{i}}$ (i.e. the absolute minimum delay of the SNIa progenitors), or the time elapsed from the end of the burst: $t-\Delta t$. Taking this into account, and neglecting the temporal dependence of the factor $A_{\text {Ia }}$, Eq. (4) becomes:

$\dot{n}_{\mathrm{Ia}}(t)=k_{\alpha} \cdot A_{\mathrm{Ia}} \cdot \psi_{0} \cdot \int_{\max \left(\tau_{\mathrm{i}}, t-\Delta t\right)}^{\min \left(t, \tau_{\mathrm{x}}\right)} f_{\mathrm{Ia}}(\tau) \mathrm{d} \tau$.

The two options for the lower integration limit correspond to two different regimes:

1. when $\tau_{\mathrm{i}}>t-\Delta t$, i.e. $t<\tau_{\mathrm{i}}+\Delta t$, we basically map the SNIa rate while the SF is still ongoing;

2. when $\tau_{\mathrm{i}}<t-\Delta t$, i.e. $t>\tau_{\mathrm{i}}+\Delta t$, we get the SNIa rate after the burst is completed.

The transition between regime 1) and 2) occurs at older ages when the SF episode lasts longer (i.e. when $\Delta t$ is larger). For a currently ongoing SF the transition has not yet occurred.

In regime 1), relevant for late type galaxies, the SNIa rate is given by:

$\dot{n}_{\mathrm{Ia}}^{L}(t)=k_{\alpha} \cdot A_{\mathrm{Ia}} \cdot \psi_{0} \cdot \int_{\tau_{\mathrm{i}}}^{\min \left(t, \tau_{\mathrm{x}}\right)} f_{\mathrm{Ia}}(\tau) \mathrm{d} \tau$

showing that it increases with time, as systems with increasingly longer delays contribute to the explosions, up to $t=\tau_{\mathrm{x}}$. From then on the SNIa rate stays constant, and equal to

$\dot{n}_{\mathrm{Ia}}^{L}(t)=k_{\alpha} \cdot A_{\mathrm{Ia}} \cdot \psi_{0}$

since $f_{\text {Ia }}$ is normalized to 1 . Notice that this expression is valid only when $\Delta t \geq \tau_{\mathrm{x}}$, that is when the SF episode lasts long enough to include all the possible delay times. In this case the current SNIa rate gives indications on the realization probability $A_{\text {Ia }}$. For example, Cappellaro et al. (1999) report an observed SNIa rate in late type galaxies of $\simeq 0.2 \mathrm{SNu}^{2}$, i.e. $0.2 \cdot\left(L_{\mathrm{B}} / L_{\mathrm{B}, \odot}\right) \times 10^{-12}$ events per year. Then, approximating

\footnotetext{
${ }^{2} 1 \mathrm{SNu}$ is one event per century per $10^{10} L_{\mathrm{B}, \odot}$.
}

$\psi_{0}$ with the ratio between the galaxy's stellar mass $(\mathcal{M})$ and its age $(t)$ Eq. (8) yields:

$A_{\mathrm{Ia}} \sim 10^{-3} \times \frac{0.2}{k_{\alpha}} \times\left(\frac{\mathcal{M}}{L_{\mathrm{B}}}\right)^{-1} \times t_{\mathrm{Gyr}}$

with $L_{\mathrm{B}}$ in solar units. Notice that the mass $\mathcal{M}$ in Eq. (9) corresponds to the integrated star formation rate, and then it differs from the actual galaxy stellar mass because of the gas returned by the stars as they evolve. Using Maraston $(1998,2005)$ simple stellar population models with solar metallicity, I find that for a constant SFR the $\mathcal{M} / L_{\mathrm{B}}$ ratio at an age of $12 \mathrm{Gyr}$ is $\sim 2$ or 1.5 , respectively for a Salpeter and Kroupa IMF. By inserting these values into Eq. (9) coupled with the appropriate $k_{\alpha}$, I get $A_{\text {Ia }} \sim 5 \times 10^{-4}$ or $10^{-3}$, respectively for Salpeter and Kroupa IMF. Thus, one SNIa event is produced for every 2000 (1000) $M_{\odot}$ of stars formed with a Salpeter (Kroupa) initial mass distribution. Although this is only a rough estimate, it appears that the current rates measured in late type systems require relatively small realization probabilities of the SNIa channel. For comparison, the realization probability of SNII, estimated as the fraction of stars more massive than $8 M_{\odot}$ in a stellar population, is $\sim 0.003,0.007$ respectively for Salpeter and Kroupa IMF. Therefore, out of a stellar population of $1000 M_{\odot}$ roughly $1 \mathrm{SNIa}$, and $7 \mathrm{SNII}$ are produced.

Considering now regime 2), relevant for early type galaxies, as long as $t<\tau_{\mathrm{x}}$ Eq. (6) yields:

$$
\begin{aligned}
\dot{n}_{\mathrm{Ia}}^{E}(t) & =k_{\alpha} \cdot A_{\mathrm{Ia}} \cdot \psi_{0} \cdot \Delta t \cdot\left\langle f_{\mathrm{Ia}}\right\rangle_{t-\Delta t, t} \\
& =k_{\alpha} \cdot A_{\mathrm{Ia}} \cdot \mathcal{M} \cdot\left\langle f_{\mathrm{Ia}}\right\rangle_{t-\Delta t, t}
\end{aligned}
$$

where $\left\langle f_{\mathrm{Ia}}\right\rangle_{t-\Delta t, t}$ is the average of the distribution function of the delay times in the range $(t-\Delta t ; t)$.

After the burst is completed, the SNIa rate scales according to the $f_{\text {Ia }}$ function, and goes to zero at $t=\tau_{\mathrm{x}}+\Delta t$. Early type galaxies are currently in this age regime, so that their SNIa rate yields information on the value of the distribution function of the delay times at ages in the vicinity of the galactic age. This constraint is better illustrated by constructing the ratio of the SNIa rate in early and late type systems at the current epoch. Dividing Eqs. (10) by (7), and considering the rates in $\mathrm{SNu}$, we get:

$\mathcal{R}_{\mathrm{SNu}}=\frac{\dot{n}_{\mathrm{Ia}, \mathrm{SNu}}^{E}}{\dot{n}_{\mathrm{Ia}, \mathrm{SNu}}^{L}} \simeq \frac{\left(\mathcal{M} / L_{\mathrm{B}}\right)^{E}}{\left(\mathcal{M} / L_{\mathrm{B}}\right)^{L}} \times \frac{\left\langle f_{\mathrm{Ia}}\right\rangle_{t-\Delta t, t}}{\left\langle f_{\mathrm{Ia}}\right\rangle_{\tau_{\mathrm{i}}, t}}$,

having assumed that the IMF and the $A_{\text {Ia }}$ factors are the same in early and late type galaxies. According to Cappellaro et al. (1999), the current epoch SNIa rate in early and late type systems is the same. This puts a constraint on the ratio between the average values of the function $f_{\mathrm{Ia}}$ :

$\frac{\left\langle f_{\mathrm{Ia}}\right\rangle_{t-\Delta t, t}}{\left\langle f_{\mathrm{Ia}}\right\rangle_{\tau_{\mathrm{i}}, t}} \simeq \frac{\left(\mathcal{M} / L_{\mathrm{B}}\right)^{L}}{\left(\mathcal{M} / L_{\mathrm{B}}\right)^{E}}$

Maraston $(1998,2005)$ models of simple stellar populations at an age of 12 Gyr and solar metallicity have $\mathcal{M} / L_{\mathrm{B}} \sim 13,10$ respectively for Salpeter and Kroupa IMF, again having considered the mass initially transformed into stars ${ }^{3}$. Combining

\footnotetext{
${ }^{3}$ Notice that at $12 \mathrm{Gyr}, \sim 30 \%$ of the initial mass has been returned to the interstellar medium by the evolving stars, for a Salpeter IMF.
} 
this estimate of the $\mathcal{M} / L_{\mathrm{B}}$ ratio for early type systems with the values quoted above for the late type systems, it turns out that the current SNIa rates indicate:

$$
\frac{\left\langle f_{\mathrm{Ia}}\right\rangle_{t-\Delta t, t}}{\left\langle f_{\mathrm{Ia}}\right\rangle_{\tau_{\mathrm{i}}, t}} \sim 0.15 \text {. }
$$

Thus the value of $f_{\text {Ia }}$ at late delay times is substantially smaller than its average value over the whole range $\left(\tau_{\mathrm{i}} ; t\right)$; in other words, the distribution function of the delay times decreases with time. This means that the majority of SNIa precursors are relatively short lived.

It is worth emphasizing that if the distribution of the delay times were flat (i.e. if young and old systems were equally efficient in producing SNIa events), the observed ratio $\mathcal{R}_{\mathrm{SNu}}$ would be of the order of 5-10, given the ratio of the $\mathcal{M} / L_{\mathrm{B}}$ values for early and late type galaxies. Therefore, the fact that the $f_{\mathrm{Ia}}$ function must be decreasing with increasing delay time is a very robust conclusion.

\subsection{Stellar evolution predictions}

The distribution function of the delay times and the realization probability of the SNIa channel from a stellar generation can be derived from the theory of the evolution of binary systems. As mentioned in the Introduction, in the literature there are several examples of the SNIa rate in stellar systems computed with population synthesis techniques (see Yungelson 2004). Typically, these models predict a $f_{\text {Ia }}$ function characterized by an early maximum, and a late epoch decline, while the realization probability is indeed on the order of $10^{-3}$. However, these results depend on the adopted parameters of the simulations, like the star formation history, the distribution functions of the separations and masses of the primordial binaries, and on the specific prescriptions for the evolution during the hydrodynamical phases of the mass transfer. Therefore, (i) the resulting $f_{\text {Ia }}$ functions are model dependent; (ii) the role of the various input parameters on the output is far from straightforward.

On the other hand, on general grounds, Ciotti et al. (1991) give a motivation for the late epoch decline related to the temporal behavior of the clock of the explosions. Indeed, some general considerations can be made which strongly characterize the shape of the distribution function of the delay times, as I show in the following sections. I consider separately the two main categories of SD and DD progenitors, and derive analytical formulations for the $f_{\mathrm{Ia}}(\tau)$ function, in the attempt of clarifying the role of the important stellar evolution parameters.

\section{Single degenerates}

In the SD model, the clock of the supernova event is set by the evolutionary lifetime of the secondary. A fit to Girardi et al. (2000) solar metallicity tracks yields the following relation between stellar mass (in $M_{\odot}$ ) and Main Sequence (MS)

The return fraction is instead $45 \%$ if Kroupa IMF applies. Therefore, the ratio between the current stellar mass and the blue luminosity predicted by the models is $\sim 9,5$ for the two IMFs.

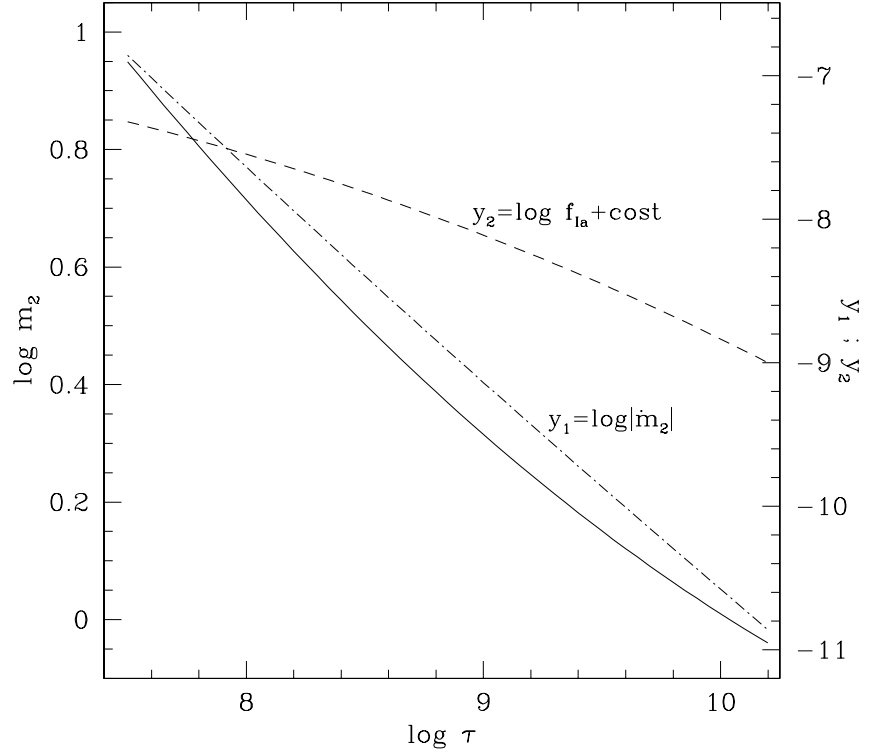

Fig. 1. Evolutionary mass (solid line) and its derivative (dot-dashed line) as a function of the MS lifetime (in years), for solar metallicity tracks. The derivative (to be read on the right axis) drops by $\sim 4$ orders of magnitude as the MS lifetime increases from $\sim 40 \mathrm{Myr}$ to $\sim 20 \mathrm{Gyr}$. The dashed line represents the distribution function of the delay times if the secondaries follow a Sapeter IMF (see text).

lifetime $\left(\tau_{\mathrm{MS}}\right.$, in years), valid in the range $\left(0.8 \lessgtr m_{2} \lesssim 8\right) M_{\odot}$, which corresponds to $\left(0.04 \lesssim \tau_{\mathrm{MS}} \lesssim 25\right) \mathrm{Gyr}$ :

$\log m_{2}=0.0471 \times\left(\log \tau_{\mathrm{MS}}\right)^{2}-1.2 \times \log \tau_{\mathrm{MS}}+7.3$.

The total delay time is basically equal to the MS lifetime of the secondary component of the binary system, the Post-MS phase being in any case much shorter than the hydrogen burning lifetime. Therefore, for one stellar generation, the number of explosions within $(\tau, \tau+\mathrm{d} \tau)$ is proportional to the number of binaries with secondary mass between $m_{2}$ and $m_{2}+\mathrm{d} m_{2}$ such that the evolutionary lifetime of $m_{2}$ is $\tau$ :

$f_{\mathrm{Ia}}^{\mathrm{SD}}(\tau)|\mathrm{d} \tau| \propto n\left(m_{2}\right)\left|\mathrm{d} m_{2}\right|$,

which implies:

$f_{\mathrm{Ia}}^{\mathrm{SD}}(\tau) \propto n\left(m_{2}\right) \cdot\left|\dot{m}_{2}\right|$.

Figure 1 shows the evolutionary mass from Eq. (12) and its derivative as a function of the delay time $\tau=\tau_{\mathrm{MS}}$. The derivative is very well approximated by the power law $\left|\dot{m}_{2}\right| \propto \tau^{-1.44}$ over the whole range from 40 Myr to $\gtrsim 15$ Gyr. In Eq. (14), as $\tau$ increases, the factor $\left|\dot{m}_{2}\right|$ strongly decreases. However, longer delay times correspond to smaller evolutionary masses $m_{2}$, and the final shape of the distribution function depends on the distribution of secondary masses as well. The dashed line in Fig. 1 shows the result obtained with a Salpeter distribution $n\left(m_{2}\right) \propto m_{2}^{-2.35}$, clearly not steep enough to imply a $f_{\mathrm{Ia}}^{\mathrm{SD}}(\tau)$ increasing at late delay times ${ }^{4}$.

However, while the distribution function of the secondary masses of primordial binaries could well be represented by

\footnotetext{
4 Since $m_{2} \propto \tau^{-0.3}$ at ages older than $1 \mathrm{Gyr}$, mass distributions as steep as $n\left(m_{2}\right) \propto m_{2}^{-4.5}$ are required in order to have a flat $f_{\mathrm{Ia}}^{\mathrm{SD}}$ at late $\tau$.
} 
a Salpeter IMF, the distribution $n\left(m_{2}\right)$ in Eq. (14) refers to the secondaries in systems which eventually give rise to a SNIa event, and as such suffers from some limitations. This is illustrated in the next section.

\subsection{The distribution function of the secondary masses}

The commonly adopted scheme in the population synthesis computations deals with binary systems in which the primaries follow a power law distribution with slope $-\alpha$, and the mass ratios $q=m_{2} / m_{1}$ follow a power law distribution with a slope $\gamma$. Then, the number of binaries with primary masses in $\left(m_{1} ; m_{1}+\mathrm{d} m_{1}\right)$ and secondaries in $\left(m_{2} ; m_{2}+\mathrm{d} m_{2}\right)$ is:

$n\left(m_{1}, m_{2}\right) \mathrm{d} m_{1} \mathrm{~d} m_{2}=n\left(m_{1}\right) f(q) \mathrm{d} m_{1} \mathrm{~d} q$.

The distribution function of the secondaries in SNIa progenitor systems, is obtained by summing over all possible primaries, ranging from a minimum value $\left(m_{1, \mathrm{i}}\right)$ to $8 M_{\odot}$ :

$$
\begin{aligned}
n\left(m_{2}\right) & \propto \int_{m_{1, \mathrm{i}}}^{8} m_{2}^{\gamma} m_{1}^{-(\alpha+\gamma+1)} \mathrm{d} m_{1} \\
& \propto m_{2}^{-\alpha} \cdot\left[\left(m_{2} / m_{1, \mathrm{i}}\right)^{\alpha+\gamma}-\left(m_{2} / 8\right)^{\alpha+\gamma}\right] .
\end{aligned}
$$

The minimum mass for the primary $\left(m_{1, \mathrm{i}}\right)$ is either equal to $m_{2}$ (as in any binary system), or more massive than this, if the primary has to produce a sufficiently massive CO WD in order to lead to a SNIa explosion. Thus:

$m_{1, \mathrm{i}}=\max \left(m_{2}, m_{1, \mathrm{n}}\right)$

where $m_{1, \mathrm{n}}$ is the mass of the primary following a specific constraint on the minimum mass of the CO WD. The restriction of the integration to systems with primaries more massive than $m_{1, \mathrm{n}}$ has important consequences on the distribution function of the delay times, and then, on the SNIa rate past a burst of SF, as will be seen in the following. For Chandrasekhar explosions, $m_{1, \mathrm{n}}$ is derived by requiring that

$m_{\mathrm{WD}, \mathrm{n}}+\epsilon \cdot m_{2, \mathrm{e}}=1.4$

where $m_{\mathrm{WD}, \mathrm{n}}$ is the minimum acceptable mass for the WD, $m_{2, \mathrm{e}}$ is the envelope mass of the evolving secondary, and $\epsilon$ is an efficiency parameter. In principle $m_{2, \mathrm{e}}$ varies with the evolutionary stage at which the second Roche Lobe Overflow (RLO) occurs, and then depends on the separation of the binary system. In practice, since the second mass transfer will not necessarily occur with $100 \%$ efficiency, this detail can be neglected, to consider a representative relation between $m_{2}$ and $m_{2, \mathrm{e}}$. For example, the results of case B RLO in Nelemans et al. (2001) can be represented by the following relation between the initial mass $\left(m_{2}\right)$ and its remnant $\left(m_{2, \mathrm{c}}\right)$ :

$m_{2, \mathrm{c}}=\max \left\{0.3 ; 0.3+0.1\left(m_{2}-2\right) ; 0.5+0.15\left(m_{2}-4\right)\right\}$.

By considering $m_{2, \mathrm{e}}=m_{2}-m_{2, \mathrm{c}}$ it turns out that smaller secondaries typically have smaller envelopes to donate to their WD companion. As a consequence, the minimum acceptable WD mass $m_{\mathrm{WD}, \mathrm{n}}$ increases as $m_{2}$ decreases, and so does the mass of its progenitor $m_{1, \mathrm{n}}$. An additional lower limit on the mass of the primary comes from the requirement that its remnant should be a CO, rather then a helium, WD. Stars less massive than $\sim 2 M_{\odot}$ develop a degenerate helium core, and evolve along the Red Giant Branch up to the helium flash, which occurs when the stellar radius is of a few hundred $R_{\odot}$ (see e.g. Fig. 1 in Yungelson 2004). Therefore, these stars can provide CO WDs only if the separation of the primordial binary exceeds $\sim 400 R_{\odot}$. Since the distribution function of the primordial separations $A_{0}$ scales as $A_{0}^{-1}$ (see, e.g. Iben $\&$ Tutukov 1984) systems with a primary less massive than $2 M_{\odot}$ are much more likely to produce a helium rather than a CO WD. For this reason, the contribution to SNIa from systems with $m_{1}$ smaller than $2 M_{\odot}$ is neglected here, and I consider:

$m_{1, \mathrm{n}}=\max \left\{2 ., 2 .+10 .\left(m_{\mathrm{WD}, \mathrm{n}}-0.6\right)\right\}$

where the relation between $m_{1, \mathrm{n}}$ and its remnant $m_{\mathrm{WD}, \mathrm{n}} \mathrm{de}-$ scribes the results of case C RLO in Nelemans et al. (2001), and represents very well the empirical determination of the initial-final mass relation by Williams et al. (2004).

In summary, as $m_{2}$ decreases, the lower limit of integration in Eq. (16) is first set to $m_{1, i}=m_{2}$, down to $m_{2}=2 M_{\odot}$; then it stays constant and equal to $2 M_{\odot}$ until the minimum WD mass for a successful SNIa event $\left(m_{\mathrm{WD}, \mathrm{n}}\right.$ from Eq. (17)) becomes larger than $0.6 M_{\odot}$. From that point on, $m_{1, \mathrm{i}}$ increases with decreasing $m_{2}$. At some value of $m_{2}, m_{1, \mathrm{i}}$ becomes equal to $8 M_{\odot}$ : this marks the minimum secondary mass suitable for a successful SNIa event.

The scheme adopted in Greggio \& Renzini (1983) is slightly different from the one just described. It assumes that the total mass of the primordial binary $M_{\mathrm{b}}$ follows a power law with slope $-\alpha$, and that the mass ratio $\mu=m_{2} / M_{\mathrm{b}}$ is distributed according to $f(\mu) \propto \mu^{\gamma}$. In this case:

$$
\begin{aligned}
n\left(m_{2}\right) & \propto \int_{M_{\mathrm{b}, \mathrm{i}}}^{M_{\mathrm{b}, \mathrm{s}}} m_{2}^{\gamma} M_{\mathrm{b}}^{-(\alpha+\gamma+1)} \mathrm{d} M_{\mathrm{b}} \\
& \propto m_{2}^{-\alpha} \cdot\left[\left(2 m_{2} / M_{\mathrm{b}, \mathrm{i}}\right)^{\alpha+\gamma}-\left(2 m_{2} / M_{\mathrm{b}, \mathrm{s}}\right)^{\alpha+\gamma}\right]
\end{aligned}
$$

where $M_{\mathrm{b}, \mathrm{s}}=8+m_{2}$ and $M_{\mathrm{b}, \mathrm{i}}=m_{1, \mathrm{i}}+m_{2}$.

It is worth remarking that in the original formulation by Greggio \& Renzini (1983), $M_{\mathrm{b}, \mathrm{i}}$ was required to be larger than a minimum value (e.g. $3 M_{\odot}$ ) irrespectively of the mass of the evolving secondary. This fixed limit does not describe the one to one correspondence between the mass of the evolving secondary and delay time of the SNIa precursor. At given delay time, the amount of mass that can be donated to the accreting white dwarf is virtually fixed, which implies a minimum white dwarf mass, in order to add up to the Chandrasekhar limit. A revision of the Greggio \& Renzini (1983) SNIa rate has been presented in Greggio (1996).

Equations (16) and (20) are very similar, but not quite the same. In both cases, the distribution function of the secondaries follows a power law with slope $-\alpha$, corrected by a factor which describes the limitations imposed on the masses of the primaries in order to secure the SNIa explosion. As $\tau$ increases, $m_{2}$ decreases and, as long as $m_{1, i}=m_{2}$, the correction factor increases toward unity. As soon as $m_{1, \mathrm{i}}$ becomes greater than $m_{2}$, the correction factor starts decreasing, due to the loss of systems with primary mass between $m_{2}$ and $m_{1, \mathrm{i}}$. 


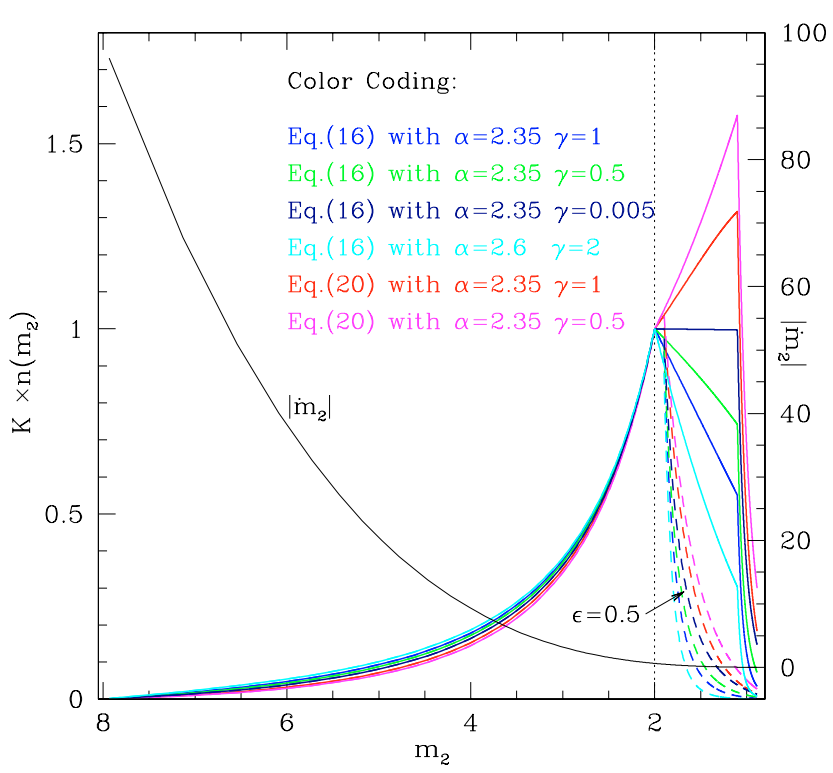

Fig. 2. The distribution function of the secondary masses in the SD model, for Chandra exploders as obtained with Eqs. (16) and (20) for selected values of the $\alpha$ and $\gamma$ parameters. Solid lines have been obtained with $\epsilon=1$, dashed lines with $\epsilon=0.5$ in Eq. (17). The black curve shows the derivative of $m_{2}$ on a linear scale (right axis).

Figure 2 shows the distribution (in arbitrary units) of the secondaries in systems which lead to Chandrasekhar explosions, under a variety of hypothesis for the power law slopes $\alpha$ and $\gamma$, and for the two formulations given by Eqs. (16) and (20), as labeled.

The distribution function of the secondaries in systems which produce a SNIa is shaped after the behavior of the minimum mass for the primary as $m_{2}$ decreases: it shows a first abrupt change of the slope when $m_{2}$ drops below $2 M_{\odot}$, that is when $m_{1, \mathrm{i}}$ is set to $2 M_{\odot}$ independently of $m_{2}$. A second abrupt change of the slope appears when the envelope mass of the secondary is so small that $m_{1, \mathrm{i}}>2 M_{\odot}$ is required in order to build up to the Chandrasekhar mass. From this point on, the distribution function of the secondaries steeply decreases as $m_{1, \mathrm{i}}$ increases. Smaller values for $\epsilon$ imply an earlier occurrence of this regime, which in the illustrated case $(\epsilon=0.5)$ appears soon after $m_{2}$ has gone below $2 M_{\odot}$.

For the same values of $\alpha$ and $\gamma$, the Greggio \& Renzini (1983) formulation (red curves) yields a larger fraction of systems with low secondary mass, compared to the formulation generally adopted in the population synthesis codes. As a result it leads to comparatively larger rates at late epochs.

The distribution $n\left(m_{2}\right)$ also appears very sensitive to the $\alpha$ and $\gamma$ parameters. Most noticeably, the flatter $\gamma$, the larger the fraction of systems with small secondaries. Figure 2 also shows the time derivative of $m_{2}$ : the combination of the two factors clearly produces an early maximum for the $f_{\mathrm{Ia}}^{\mathrm{SD}}$ function which is given by Eq. (14).

\subsection{The distribution function of the delay times}

Figure 3 shows the resulting distribution function of the delay times, with the same color and line coding as in Fig. 2.

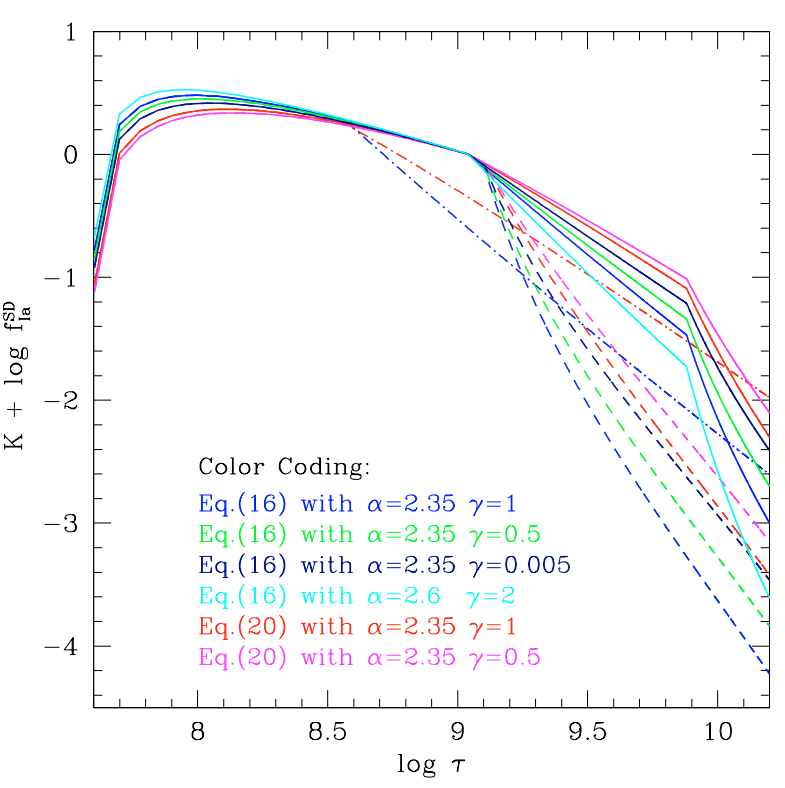

Fig. 3. Distribution function of the delay times for the SD model for the same choices of the parameters as in Fig. 2. In addition, the dotdashed lines show the distribution function for the Sub-Chandra models in the case $(\alpha, \gamma)=(2.35,1)$, using Eqs. (16) (blue) and (20) (red).

The two abrupt changes of the slope just reflect those appearing in the $n\left(m_{2}\right)$ function. Figure 3 also shows the distribution functions of the delay times expected for the Sub-Chandra exploders as dot-dashed lines. These are obtained from Eqs. (16) and (20) with the following criterion for $m_{1, \mathrm{i}}$ : a minimum WD mass of $0.7 M_{\odot}$ is required to secure the explosion, along the lines suggested by Woosley \& Weaver (1994) models. This corresponds to a minimum primary mass of $3 M_{\odot}$ (see the second term of the RHS in Eq. (19)), so that the first cusp occurs as early as 0.4 Gyr. In order to have a Sub-Chandra SNIa, a helium layer of about $0.15 M_{\odot}$ needs to be accumulated on top of the CO WD, which corresponds to $\left(\epsilon \cdot m_{2, \mathrm{e}}\right) \geq 0.15 M_{\odot}$. This is a limit on $m_{2}$ which implies that the single burst SNIa rate for this model drops to zero at a delay time equal to the evolutionary lifetime of such $m_{2}$. Since the evolutionary mass at 15 Gyr is $\sim 0.9 M_{\odot}$, this limit is inactive for the whole Hubble time, provided that $\epsilon \gtrsim 0.3$, as is considered for Fig. 3 .

The distribution function of the delay times for the SD model is basically shaped according to the limits on the mass of the primaries in systems which eventually produce a SNIa. Four regimes can be identified:

1) Regime A, in which $f_{\mathrm{Ia}}^{\mathrm{SD}}$ shows an initial steep rise followed by a mild decrease. In this regime $m_{1, i}=m_{2}$, implying that at longer delay times there is an increasing range of $m_{1}$ which contribute to the SNIa explosions. The (wide) maximum results from the interplay between $n\left(m_{2}\right)$ and $\left|\dot{m}_{2}\right|$, the latter being a decreasing function of $\tau$.

2) Regime $\mathrm{B}$, which sets in when $m_{2}$ falls below the minimum primary mass suitable for the explosion. Such minimum mass is chosen here equal to 2 and $3 M_{\odot}$ respectively for Chandra and Sub-Chandra exploders. In Regime B, $m_{1, \mathrm{i}}$ is kept constant and equal to this minimum primary mass; the distribution function of the delay times decreases with 
a steeper slope compared to Regime A, due to the loss of systems with $m_{2} \leq m_{1} \leq 2$. It can be shown that in this regime, the $f_{\mathrm{Ia}}^{\mathrm{SD}}$ function is mostly sensitive to the parameter $\gamma$, according to $\log f_{\mathrm{Ia}}^{\mathrm{SD}} \propto \tau^{-1.44-0.3 \gamma}$.

3) Regime C, starting when primaries more massive than $2 M_{\odot}$ are required in order to build up to the Chandrasekhar mass. In Regime $\mathrm{C}, m_{1, \mathrm{i}}$ increases from 2 to $8 M_{\odot}$ as $\tau$ increases; consequently $f_{\mathrm{Ia}}^{\mathrm{SD}}$ shows a steep decline. For Sub-Chandra exploders Regime $\mathrm{C}$ does not exist.

4) Regime $\mathrm{D}$, in which $f_{\mathrm{Ia}}^{\mathrm{SD}}=0$ : this sets in when $m_{1, \mathrm{i}}=8 M_{\odot}$ is required to produce a Chandrasekhar explosion, or when the envelope mass of $m_{2}$ drops below the $(0.15 / \epsilon)$ limit. Regime D is not shown in Fig. 3. The value of $\tau$ at which this regime sets in defines $\tau_{\mathrm{x}}$ for the SD model.

For the Chandra exploders, the delay time at which Regime C sets in varies with the efficiency of the accretion process. The response of the CO WD to accretion crucially depends on the accretion rate, as illustrated in Hachisu \& Kato (2001). Only if the accretion rate is in a small range around $10^{-7} M_{\odot} / \mathrm{yr}$ does steady burning occur on top of the WD, so that the mass of the donor is efficiently used to increase the mass of the WD. For both lower and higher accretion rates, part of the donated mass is lost, either following $\mathrm{H}$-shell flashes (novae explosion), for low accretion rates, or in a thick wind, for high accretion rates. Therefore, the accretion efficiency, i.e. the ratio between the accreted and the donated matter, is unlikely to be close to unity, as adopted for the solid lines in Fig. 3. The lower the accretion efficiency, the earlier regime $\mathrm{C}$ sets in, and regime $\mathrm{B}$ is suppressed when $\epsilon \lesssim 0.5$, as shown by the dashed lines in Fig. 3.

The shape of the $f_{\mathrm{Ia}}^{\mathrm{SD}}$ function derived here is quite general, since it just reflects the product of the time derivative of the evolutionary secondary mass, and the distribution $n\left(m_{2}\right)$.

\section{Double degenerates}

In the DD model, the first part of the close binary evolution is the same as in the SD model, but, following the expansion of the secondary component, a Common Envelope phase (CE) sets in, eventually leading to the complete ejection of the CE itself. In this scenario, accretion on top of the WD, if any, is neglected. The system emerges from the CE as a close binary composed of two WDs, which are bound to eventually merge due to the emission of gravitational wave radiation. If the total mass of the DD system exceeds the Chandrasekhar mass, explosion occurs as a SNIa event. The delay time in this case is $\tau=\tau_{\mathrm{n}}+\tau_{\mathrm{gw}}$

where $\tau_{\mathrm{n}}$ is the nuclear lifetime of the secondary, and $\tau_{\mathrm{gw}}$ is the gravitational delay (Landau \& Lifshitz 1962):

$\tau_{\mathrm{gw}}=\frac{0.15 A^{4}}{\left(m_{1 \mathrm{R}}+m_{2 \mathrm{R}}\right) m_{1 \mathrm{R}} m_{2 \mathrm{R}}} \mathrm{Gyr}$

$A, m_{1 \mathrm{R}}$ and $m_{2 \mathrm{R}}$ being respectively the separation and component masses of the DD system, in solar units.

To derive the distribution function of the delay times one needs to map the distribution of the primordial systems in the space $\left(A_{0}, m_{1}, m_{2}\right)$ into that of the final systems in the space $(A$, $\left.m_{1 \mathrm{R}}, m_{2 \mathrm{R}}\right)$. Rather then performing Montecarlo simulations I consider here some general aspects, with the aim of characterizing the shape of the $f_{\text {Ia }}$ function.

I restrict to systems with $2 \leq m_{1}, m_{2} \leq 8$, from which most double CO WDs form, as argued in Sect. 3.1. Typically, the WD mass of both components ranges between 0.6 and $1.2 M_{\odot}$ (see e.g. Eq. (19)), and $\tau_{\mathrm{n}}$ ranges between 0.04 and $1 \mathrm{Gyr}$. The gravitational delay spans a large range, depending on the final separation: e.g., for a $(0.7+0.7) M_{\odot} \mathrm{DD}, \tau_{\mathrm{gw}}$ increases from 0.014 to $18 \mathrm{Gyr}$ when $A$ goes from 0.5 to $3 R_{\odot}$. The distribution function of the delay times will depend on the distributions of both $\tau_{\mathrm{n}}$ and $\tau_{\mathrm{gw}}$, at least up to total delays of $\sim$ few Gyr, with early explosions provided by systems with short $\tau_{\mathrm{n}}$ and $\tau_{\mathrm{gw}}$. Since the maximum nuclear delay does not exceed $\sim 1 \mathrm{Gyr}$, at long $\tau$ the SNIa events will come from systems with long gravitational delays, that is DDs with wide separations and low masses.

\subsection{The interplay of $\tau_{n}$ and $\tau_{g w}$}

The following useful approximation for the gravitational delay is justified in Appendix A.1:

$\tau_{\mathrm{gw}}=0.6 \frac{A^{4}}{M_{\mathrm{DD}}^{3}} \mathrm{Gyr}$

where $M_{\mathrm{DD}}$ is the total mass of the DD system (again in solar units). This formula results from considering the restrictions on the masses of the two WD components. In this approximation the gravitational delay does not explicitly depend on $m_{2 R}$. Since $\tau_{\mathrm{n}}$ only depends on $m_{2}$, which is in tight correspondence with $m_{2 \mathrm{R}}$, the relation between the two timescales is very weak: basically, primordial systems with secondary mass $m_{2}$ will evolve into a family of SNIa precursors spanning a wide range in separations and total binary mass, that imply a wide range of gravitational delays.

The integrated distribution of the total delay times can now be constructed: the contribution $\left(\mathrm{d} n_{\mathrm{n}}\right)$ of binaries with given $\tau_{\mathrm{n}}$ to the number of systems with total delay time shorter than $\tau$ is proportional to the fraction of them which have $\tau_{\mathrm{gw}}$ shorter than $\left(\tau-\tau_{\mathrm{n}}\right)$. Indicating this quantity with $g\left(\tau, \tau_{\mathrm{n}}\right)$ :

$\mathrm{d} n_{\mathrm{n}}=n\left(\tau_{\mathrm{n}}\right) \cdot g\left(\tau, \tau_{\mathrm{n}}\right) \mathrm{d} \tau_{\mathrm{n}}$

where $n\left(\tau_{\mathrm{n}}\right)$ is the distribution function of the nuclear timescales, proportional to the $f_{\mathrm{Ia}}^{\mathrm{SD}}$ function described in Sect. 3. The total number of systems with total delay time shorter than $\tau$ is obtained by integrating the $\mathrm{d} n_{\mathrm{n}}$ contributions over the relevant $\tau_{\mathrm{n}}$ range:

$\int_{0}^{\tau} n(\tau) \mathrm{d} \tau=\int_{\tau_{\mathrm{n}, \mathrm{i}}}^{\min \left(\tau_{\mathrm{n}, \mathrm{x}}, \tau\right)} n\left(\tau_{\mathrm{n}}\right) g\left(\tau, \tau_{\mathrm{n}}\right) \mathrm{d} \tau_{\mathrm{n}}$

where $\tau_{\mathrm{n}, \mathrm{i}}$ and $\tau_{\mathrm{n}, \mathrm{x}}$ bracket the range of nuclear timescales of the systems which end up in a successful SNIa event.

Let's now indicate with $n\left(\tau_{\mathrm{gw}}\right)$ the distribution function of the gravitational delays of systems with a nuclear delay $\tau_{\mathrm{n}}$ (i.e. progeny of systems with a secondary mass $m_{2}$ whose MS lifetime is equal to $\tau_{\mathrm{n}}$ ), and let $\tau_{\mathrm{gw}, \mathrm{i}}$ and $\tau_{\mathrm{gw}, \mathrm{x}}$ be the minimum and 
maximum gravitational delays of such systems. Formally, the fraction of systems which, having a nuclear delay equal to $\tau_{\mathrm{n}}$, have also a total delay shorter than $\tau$ varies with $\tau$ according to:

$g\left(\tau, \tau_{\mathrm{n}}\right)= \begin{cases}0 & \text { for } \tau \leq \tau_{1} \\ \int_{\tau_{\mathrm{gw}, \mathrm{i}}}^{\min \left(\tau_{\mathrm{gw}, \mathrm{x}}, \tau-\tau_{\mathrm{n}}\right)} n\left(\tau_{\mathrm{gw}}\right) \mathrm{d} \tau_{\mathrm{gw}} & \text { for } \tau_{1} \leq \tau \leq \tau_{2} \\ 1 & \text { for } \tau \geq \tau_{2}\end{cases}$

with $\tau_{1}=\tau_{\mathrm{n}}+\tau_{\mathrm{gw}, \mathrm{i}}$ and $\tau_{2}=\tau_{\mathrm{n}}+\tau_{\mathrm{gw}, \mathrm{x}}$. This equation merely expresses that (i) systems with nuclear delay $\tau_{\mathrm{n}}$ have a total delay ranging between $\tau_{\mathrm{n}}+\tau_{\mathrm{gw}, \mathrm{i}}$ and $\tau_{\mathrm{n}}+\tau_{\mathrm{gw}, \mathrm{x}}$; (ii) the total delay of such systems scales according to the distribution function of their gravitational delays.

The shape of $n\left(\tau_{\mathrm{gw}}\right)$ will reflect the distribution of the final separations and of the DD masses. The interesting range of gravitational delays, which goes from $\sim 0.01$ Gyr (i.e. on the order of $\left.\tau_{\mathrm{n}, \mathrm{i}}\right)$ to over the Hubble time, is populated by low mass systems $\left(M_{\mathrm{DD}}=1.4 M_{\odot}\right)$ with $0.5 \lesssim A / R_{\odot} \lesssim 2.8$, and by high mass systems $\left(M_{\mathrm{DD}}=2.4 M_{\odot}\right)$ with $0.7 \lesssim A / R_{\odot} \lesssim 4.2$. In the next section the outcome of the close binary evolution is examined in connection to the possibility of producing final separations in the reference range $0.5 \lesssim A / R_{\odot} \lesssim 4.5$, which corresponds to the relevant range of gravitational delays, for the full mass range of the DD systems.

\subsection{Shrinkage during the mass transfer phases}

The mass transfer phase in a close binary system may be dynamically stable or unstable: in the first case the outcome is a wide system (occasionally wider than the primordial separation), and the secondary may have accreted some of the donor's envelope mass. In the second case, a CE occurs and (generally) the system shrinks. The occurrence of one evolutionary channel rather than another depends on the configuration of the initial binary (e.g. the mass ratio at RLO, whether the envelope of the donor is radiative or convective, and so on). Anyway, the interesting systems are those which suffer a substantial degree of shrinkage during their evolution: the initial separations of the double CO WD progenitors range roughly from 100 to $1000 R_{\odot}$, so that the first RLO takes place at all (upper limit), and that it does so after core helium exhaustion (lower limit), when the star is on the Asymptotic Giant Branch. Thus, in order to merge within a Hubble time, the binary evolution should produce a total shrinkage on the order of $\sim 10^{-2}, 10^{-3}$. This can be accomplished through one or more CE phases.

The standard CE recipe (e.g. Webbink 1984) relates the initial and final values of the binary parameters by requiring that the variation of the orbital energy is proportional to the binding energy of the envelope of the donor:

$\frac{m_{\mathrm{i}}^{\mathrm{d}}\left(m_{\mathrm{i}}^{\mathrm{d}}-m_{\mathrm{f}}^{\mathrm{d}}\right)}{R}=\alpha_{\mathrm{ce}}\left[\frac{m_{\mathrm{f}}^{\mathrm{d}} m}{2 A_{\mathrm{f}}}-\frac{m_{\mathrm{i}}^{\mathrm{d}} m}{2 A_{\mathrm{i}}}\right]$

where $m_{\mathrm{i}}^{\mathrm{d}}, m_{\mathrm{f}}^{\mathrm{d}}$ are the mass of the donor respectively before and after the CE, $m$ is the mass of the companion, $A_{\mathrm{i}}$ and $A_{\mathrm{f}}$ are the separations before and after the CE, $R$ is the radius of the donor at contact (i.e. the Roche Lobe radius). $\alpha_{\text {ce }}$ is a parameter roughly describing the efficiency with which the orbital energy of the binary is used to expel the $\mathrm{CE}^{5}$ : when smaller than unity the process is very inefficient, and the system emerges from the CE with a small separation; when $\alpha_{\text {ce }}>1$ other energy sources, besides the orbital one, are used to expel the CE. Typical values considered in the literature, and supported by hydrodynamical computations, range from $\alpha_{\mathrm{ce}}=0.5$ to $\alpha_{\mathrm{ce}}=2$ (Rasio \& Livio 1996).

However, this formulation fails to explain the observed binary parameters of 3 double He WDs, and Nelemans et al. (2000) propose an alternative equation for the first mass transfer, which consists in a parametrization of the system's angular momentum loss:

$\frac{J_{\mathrm{i}}-J_{\mathrm{f}}}{J_{\mathrm{i}}}=\gamma_{\mathrm{CE}} \frac{\Delta M}{M_{\mathrm{B}}}$

where $J_{\mathrm{i}}, J_{\mathrm{f}}$ are the initial and final angular momenta, $\Delta M$ is the mass lost from the system (roughly equal to the donor's envelope mass), $M_{\mathrm{B}}$ is the total mass of the binary before RLO, and $\gamma_{\mathrm{CE}}$ is a parameter, for which Nelemans et al. (2000) find a value of $\simeq 1.5$ by fitting the data. This formulation has been recently shown to describe the properties of a larger sample of binary WDs (Nelemans \& Tout 2005).

Equation (26) leads to a dramatic shrinkage, the typical ratio between the separations after and before the CE phase $\left(A_{\mathrm{f}} / A_{\mathrm{i}}\right)$ being on the order of a few $10^{-2}$; instead, for systems with a high mass ratio $q\left(=m_{2} / m_{1}\right)$, Eq. (27) leads to modest shrinkage $\left(A_{\mathrm{f}} / A_{\mathrm{i}} \sim 1\right)$. At low $q$, Eqs. (26) and (27) yield similar values, and actually, Eq. (27) is only applicable at relatively large $q$ (see Appendix A.2). As for the second mass transfer episode, Eq. (26) is generally adopted in the literature. I have then explored the results of two different evolutionary schemes: (i) the two successive RLO are regulated by Eq. (26); (ii) the first mass transfer occurs according to Eq. (27) (when applicable, see Appendix A.2 for a detailed description), while the second mass transfer is regulated by Eq. (26). The evolutionary scheme (i) corresponds to the prescriptions used by e.g. Tutukov \& Yungelson (1994), Ruiz-Lapuente \& Canal (1998), Han et al. (1995), Han (1998), Yungelson \& Livio (2000); the evolutionary scheme (ii) corresponds to the prescriptions used in Nelemans et al. (2001).

The total shrinkage, that is the ratio $\left(A / A_{0}\right)$ between the separation of the newborn DD system and the original separation of the binary, is shown in Fig. 4 as a function of the mass of the secondary component in the primordial system: blue and cyan dots refer to the (i), red and magenta dots to the (ii) evolutionary schemes. At fixed $m_{2}$ the points show the effect of varying $m_{1}$ : the more massive $m_{1}$ the smaller the $A / A_{0}$ ratio. The four panels show the effect of varying the parameters $\alpha_{\mathrm{ce}}$ and $\gamma_{\mathrm{CE}}$ in Eqs. (26) and (27). In order to gauge the effect of mass loss through stellar wind, which may occur prior to the CE phases, an additional parameter $\left(f_{\text {en }}\right)$ has been considered, such that the mass of the donor at each RLO is equal to a fraction $f_{\text {en }}$ of its initial mass. The sensitivity of the final shrinkage to this parameter proves significant.

\footnotetext{
5 Different formulations are found in the literature for Eq. (26), and correspondingly different meanings for $\alpha_{\text {ce }}$. I adopt here the formulation in Nelemans et al. (2001), with the geometrical parameter $\lambda=1$.
} 


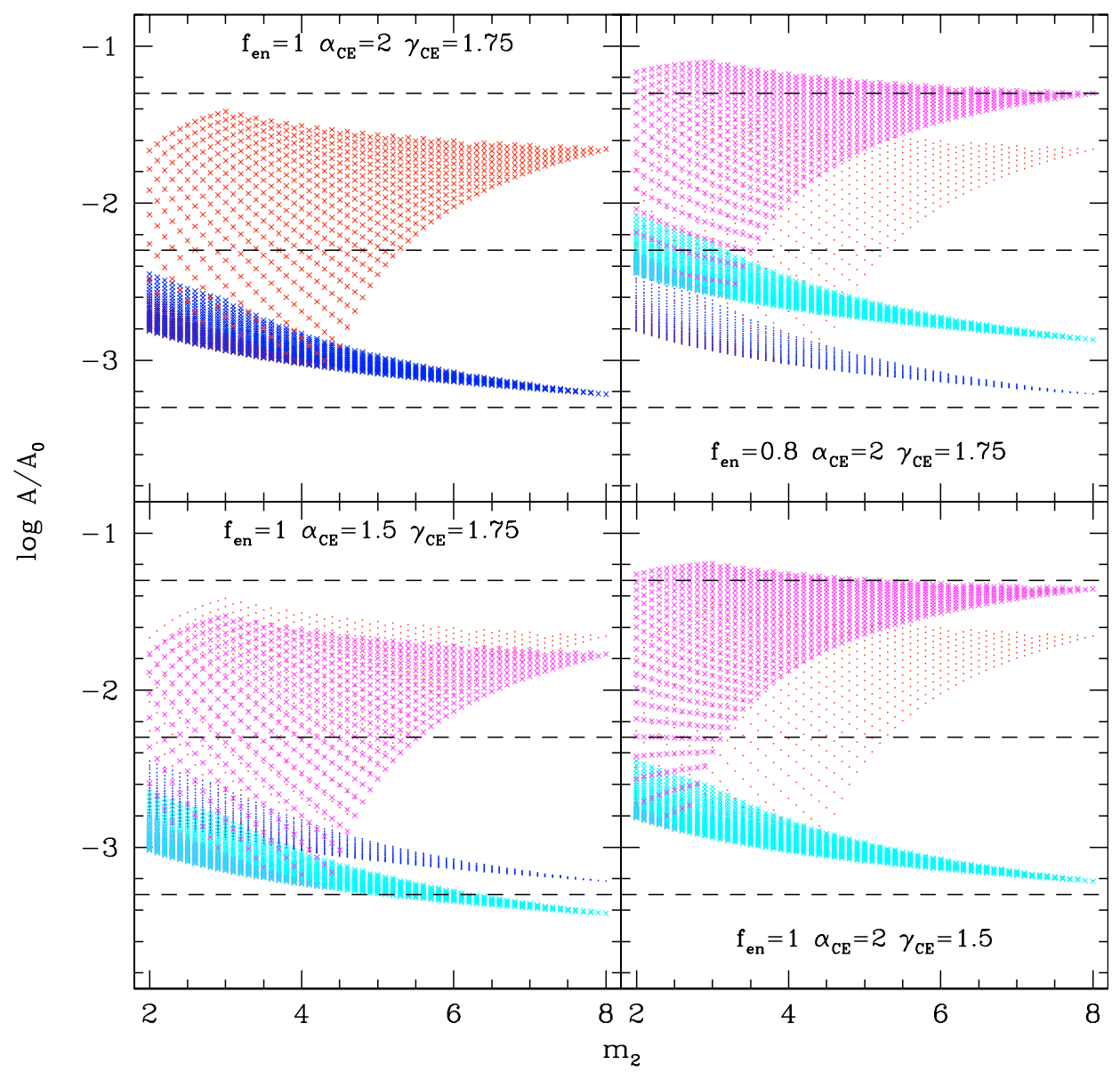

Fig. 4. Exploration of the outcome of two mass transfer episodes in binaries with intermediate mass. The ratio between the separations of the final DD and the primordial systems is plotted as a function of the initial mass of the secondary for systems with $m_{1} \geq m_{2}$. Equation (19), and the Eggleton (1998) formula for the Roche Lobe radius have been used. Blue and cyan points result from the application of Eq. (26) to both mass transfers; red and magenta points from applying either Eqs. (27) or (26) for the first mass transfer, depending on which of the two yields the wider separation $A_{\mathrm{f}}$. At given $m_{2}$, the larger $m_{1}$ the smaller $A / A_{0}$ is. Each panel is labeled with the adopted values for the parameters in Eqs. (26) and (27). The results in the top-left panel are reproduced in the other panels as small dots for an easy comparison.

The two options lead to vastly different situations: for the scheme (i) the $A / A_{0}$ ratio appears confined in a narrow range, around a mean value which depends on $\alpha_{\text {ce }}$ and $f_{\text {en }}$. In addition, there is a clear trend of $A / A_{0}$ decreasing as $m_{2}$ increases. Indeed, more energy is required to expel the more massive $\mathrm{CE}$ in the more massive binaries. At fixed $\alpha_{\text {ce }}$ this implies that the systems shrink more and end up with a smaller $A / A_{0}$ ratios. The (ii) prescriptions, instead, produce a wide range of $A / A_{0}$ ratios at almost every $m_{2}$. This means that systems with the same $m_{2}$ and $A_{0}$ can end up very wide or very close depending on the mass of the companion.

Turning now to consider the quantitative value of $A / A_{0}$, the three lines in Fig. 4 show the levels $A / A_{0}=5 \times 10^{-4}, 5 \times 10^{-3}$, $5 \times 10^{-2}$. Recalling that the initial separations of the double CO WD progenitors range roughly from 100 to $1000 R_{\odot}$, such levels correspond respectively to final separations of $\simeq 0.05,0.5$ and $5 R_{\odot}$ for the initially closest systems; to $A \simeq 0.5,5$ and $50 R_{\odot}$ for the initially widest ones. Inspection of Fig. 4 shows that the evolutionary scheme (ii) is capable of producing final separations in a very wide range, well including the range $0.5 R_{\odot} \lesssim A \lesssim 5 R_{\odot}$, leading to merging within a Hubble time.
On the contrary, the scheme (i) appears to produce very small $A / A_{0}$ ratios, so that only the initially widest binaries manage to merge on timescales on the order of some Gyr. In addition, the correlation between $A / A_{0}$ and $m_{2}$ implies that lower mass systems have longer gravitational delays, as will be better illustrated in the next section.

It's important to notice the high sensitivity of the $A / A_{0}$ ratio on the parameters $\left(\alpha_{\mathrm{ce}}, f_{\mathrm{en}}, \gamma_{\mathrm{CE}}\right)$; this, coupled with the high sensitivity of $\tau_{\mathrm{gw}}$ on $A$ suggests that the results of the binary evolution from the population synthesis codes are very dependent of the exact recipe used. At the same time, the correspondence between the $\left(A_{0}, m_{1}, m_{2}\right)$ and $\left(A, m_{1 \mathrm{R}}, m_{2 \mathrm{R}}\right)$ is likely to be rather loose, and even more so if a distribution of $\left(\alpha_{\mathrm{ce}}, f_{\mathrm{en}}, \gamma_{\mathrm{CE}}\right)$ values is realized in nature. In this respect, it is worth recalling that the computations here adopt a unique initial-final mass relation, while in reality, at a given initial mass, the remnant mass spans a (small) range, in relation to the precise point in the evolution at which the mass transfer takes place. Therefore, for each pair $\left(m_{1}, m_{2}\right)$ there will be a distribution of $A / A_{0}$, around the corresponding point in Fig. 4. 
This exploration of the results of the CE phases suggests to consider the two following extreme characterizations for the $n\left(\tau_{\mathrm{gw}}\right)$ function:

- either, irrespectively of $m_{2}$, the close binary evolution produces a wide distribution of $A$ and $M_{\mathrm{DD}}$, and these two variables are virtually independent;

- or the close binary evolution leads to a narrow distribution of the $A / A_{0}$ ratio, so that the initially closest binaries merge in a short time, and the initially widest binaries tend to populate the long $\tau_{\mathrm{gw}}$ tail of the distribution. In addition, the most massive binaries tend to end up with the smallest final separation, hence merge more quickly.

The first characterization is more appropriate for the scheme (ii) of evolution, and will be referred to as WIDE DDs; the second, suggested by the results of the evolutionary scheme (i), will be referred to as CLOSE DDs. Since both schemes provide very small $A / A_{0}$ ratios for some values of the parameters of the primordial binary system, the minimum gravitational delay $\tau_{\mathrm{gw}, \mathrm{i}}$ is likely to be very short. The dependence of the distribution function of the delay times on the parameter $\tau_{\mathrm{gw}, \mathrm{i}}$ will be explored later, and only for the WIDE DD scheme. The maximum gravitational delay $\tau_{\mathrm{gw}, \mathrm{x}}$ is instead an important parameter for the characterization of the two schemes, as shown in the next sections.

\subsection{The distribution $g\left(\tau, \tau_{n}\right)$}

The fraction of systems which, having a nuclear timescale $\tau_{\mathrm{n}}$ manage to merge within a timescale shorter than $\tau_{\mathrm{gw}}=\tau-\tau_{\mathrm{n}}$ depends on the distribution of the gravitational delays. In this section the expressions for the $g\left(\tau, \tau_{\mathrm{n}}\right)$ function are derived for the WIDE DD and CLOSE DD schemes, based on the two different characterizations.

\subsubsection{WIDE DDs}

For the WIDE DD scheme the assumptions are:

- $M_{\mathrm{DD}}$ and $A$ are independent variables;

- the minimum gravitational delay $\tau_{\mathrm{gw}, \mathrm{i}}$ is independent of $m_{2}$;

- the maximum gravitational delay $\tau_{\mathrm{gw}, \mathrm{x}}$ is larger than $\tau-\tau_{\mathrm{n}}$ for all $\tau_{\mathrm{n}}$ (at least for a total delay time up to the Hubble time);

- the distribution function of the final separations follows a power law: $n(A) \propto A^{\beta_{\mathrm{a}}}$.

Under these conditions, in Appendix A.3 the following formula is derived:

$g\left(\tau, \tau_{\mathrm{n}}\right) \propto \begin{cases}0 & \text { for } \tau \leq \tau_{1} \\ f_{1,2}^{\mathrm{W}}\left[\left(\tau-\tau_{\mathrm{n}}\right)^{\tilde{\beta}_{\mathrm{a}}}-\tau_{\mathrm{gw}, \mathrm{i}}^{\tilde{\beta}_{\mathrm{a}}}\right] & \text { for } \tau_{1} \leq \tau \leq \tau_{2}\end{cases}$

where $\tilde{\beta}_{\mathrm{a}}=0.25\left(1+\beta_{\mathrm{a}}\right), \tau_{1}=\tau_{\mathrm{n}}+\tau_{\mathrm{gw}, \mathrm{i}}, \tau_{2}=\tau_{\mathrm{n}}+\tau_{\mathrm{gw}, \mathrm{x}}$ and $f_{1,2}^{\mathrm{W}}$ is a function of $\tau_{\mathrm{n}}$, for which two cases are considered:

$f_{1,2}^{\mathrm{W}}=\left\{\begin{array}{l}f_{1}^{\mathrm{W}}=\tilde{M}_{\mathrm{DD}}^{0.75+0.75 \beta_{\mathrm{a}}} \\ f_{2}^{\mathrm{W}}=M_{\mathrm{DD}, \mathrm{x}}^{1.75+0.75 \beta_{\mathrm{a}}}-M_{\mathrm{DD}, \mathrm{n}}^{1.75+0.75 \beta_{\mathrm{a}}}\end{array}\right.$

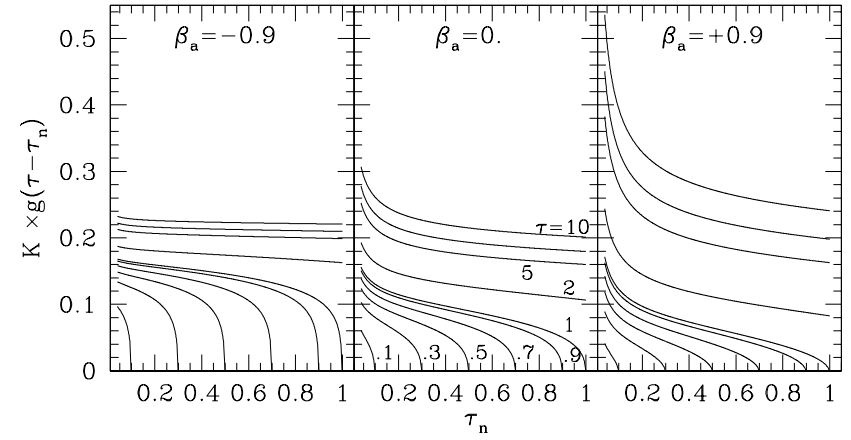

Fig. 5. The $g\left(\tau, \tau_{\mathrm{n}}\right)$ function for WIDE $D D s$, with $f_{1,2}^{\mathrm{W}}=f_{1}^{\mathrm{W}}$, for three values of the $\beta_{\text {a }}$ parameter. The functions, plotted on arbitrary units, are shown for $\tau=0.1,0.3,0.5,0.7,0.9,1,2,5,7$ and $10 \mathrm{Gyr}$. A very small minimum gravitational delay $\tau_{\mathrm{gw}, \mathrm{i}}=0.001 \mathrm{Gyr}$ has been used.

with

$M_{\mathrm{DD}, \mathrm{n}}=\max \left(1.4, m_{2 \mathrm{R}}+0.6\right) ; \quad M_{\mathrm{DD}, \mathrm{x}}=m_{2 \mathrm{R}}+1.2 ;$

$\tilde{M}_{\mathrm{DD}}=1.4+\left(m_{2}-2\right) / 6$

and $m_{2 \mathrm{R}}$ given by Eq. (19). The first case in Eq. (29) applies when there is a tight correlation between $m_{2}$ and its $M_{\mathrm{DD}}$ progeny; the second applies when a wide distribution of $M_{\mathrm{DD}}$ is obtained from systems with the same $m_{2}$.

Figure 5 illustrates the $g\left(\tau, \tau_{\mathrm{n}}\right)$ function (computed with the $f_{1}^{\mathrm{W}}$ factor). In general, as $\tau$ increases, the $g$ function increases at every $\tau_{\mathrm{n}}$, since at longer delays more systems fulfill the condition $\tau_{\mathrm{gw}}+\tau_{\mathrm{n}} \leq \tau$ at each $\tau_{\mathrm{n}}$. At the same time, for increasing $\tau_{\mathrm{n}}$ the number of systems with total delay smaller than a fixed $\tau$ decreases, partly because of the decrease of the range of gravitational delays which fulfill the condition $\tau_{\mathrm{gw}} \leq \tau-\tau_{\mathrm{n}}$. A more thorough explanation of the trend of the $g$ function can be found in Appendix A.3. For $\beta_{\mathrm{a}}=-0.9$ most DDs are born with small separations, and the dependences on both $\tau_{\mathrm{n}}$ and $\tau$ are milder, since both the above mentioned effects are less relevant. Conversely, when $\beta_{\mathrm{a}}=+0.9$ the distribution of the gravitational delays is skewed toward the large $\tau_{\mathrm{gw}}$ values: the fraction of systems with total delay up to $\tau$ greatly increases with $\tau$, as systems with longer $\tau_{\mathrm{gw}}$ are included.

It is worth pointing out that for $\beta_{\mathrm{a}}<-1$ the $g$ function is very sensitive to $\tau_{\mathrm{gw}, \mathrm{i}}$. This regime corresponds to distributions $n(A)$ highly peaked at the minimum separation, a possibility that likely provides exceedingly small SNIa rates at late epochs in early type galaxies.

\subsubsection{CLOSE DDS}

The distinctive features of the end product of the CLOSE DD scheme of evolution appear to be that (i) the average $A / A_{0}$ ratio is very small; (ii) it is correlated with the mass of the secondary in the primordial system. As a result, the gravitational delays can be very small even for the systems with maximum primordial separation, especially for the most massive DDs.

Figure 6 shows the gravitational delay in this evolutionary scheme for systems born with $A_{0}=1000 R_{\odot}$, and having adopted $\alpha_{\mathrm{ce}}=2$ and $f_{\mathrm{en}}=0.9$. Notice that these are about the 


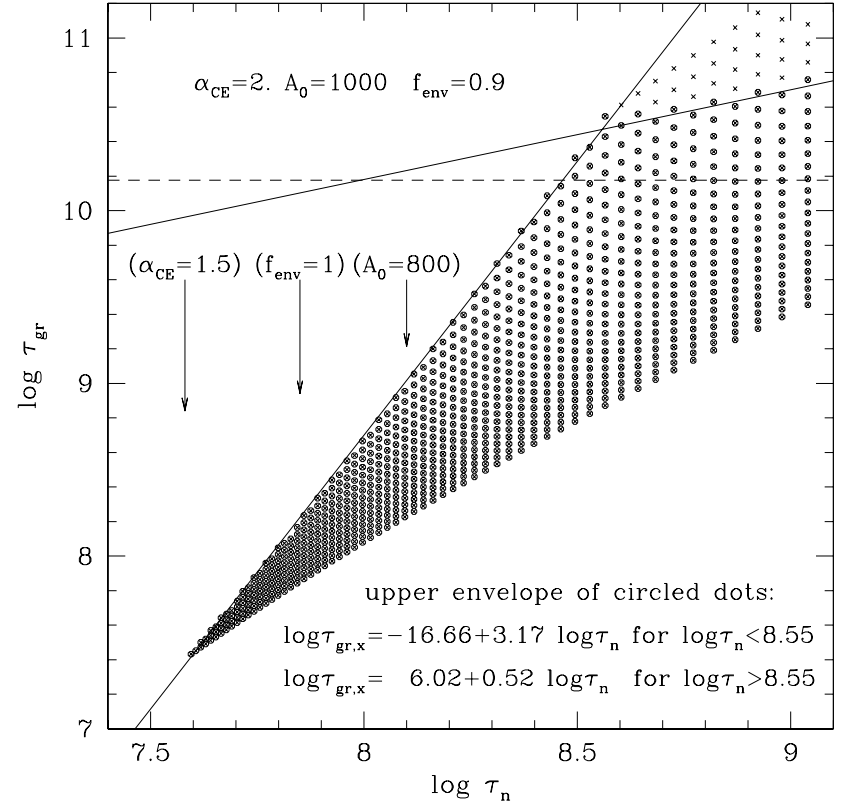

Fig. 6. Exploration of the gravitational delay of DDs from systems with a primordial separation of $A_{0}=1000 R_{\odot}$, evolved through two CE phases regulated by Eq. (26), as a function of the MS lifetime of the secondary. Both ages are expressed in years. Circled dots mark systems with $M_{\mathrm{DD}} \geq 1.4 M_{\odot}$. The dashed line shows the $15 \mathrm{Gyr}$ level; the arrows show the average shift which applies when, leaving the other parameters unchanged, one adopts in turn different values for $f_{\mathrm{en}}, \alpha_{\mathrm{ce}}$ or $A_{0}$ as specified.

widest interacting binaries: the components of systems born with a larger $A_{0}$ evolve as single stars, and do not provide SNIa, at least in a Hubble time. In Fig. 6, for every $m_{2}$ (i.e. $\tau_{\mathrm{n}}$ ), $m_{1}$ is decreased from 8 to $m_{2}{ }^{6}$, leading to longer gravitational delays ( $M_{\mathrm{DD}}=m_{1 \mathrm{R}}+m_{2 \mathrm{R}}$ decreases). The circled dots refer to systems with $M_{\mathrm{DD}} \geq 1.4$, and therefore suited to successful SNIa events. The upper envelope of the gravitational delays of the SNIa precursors is well represented by:

$$
\log \tau_{\mathrm{gw}, \mathrm{x}}=\min \left(-16.66+3.17 \log \tau_{\mathrm{n}} ; 6.02+0.52 \log \tau_{\mathrm{n}}\right)
$$

with the delay times expressed in years. By varying the parameters $\alpha_{\mathrm{ce}}$ and $f_{\mathrm{en}}$ the linear regressions shift vertically, approximately maintaining their slopes. The vertical arrows drawn in Fig. 6 show the amount of this shift for the indicated value of the parameters.

The locus $\tau_{\mathrm{gw}}=15 \mathrm{Gyr}$ is shown as a dashed line in Fig. 6: it appears that the possibility of realizing gravitational delays as long as the Hubble time is related to a fine tuning of the involved parameters, so that the shrinkage due to the two CE phases is not too severe. The systematic increase of the maximum gravitational delay with increasing nuclear lifetime of the secondary reflects the smaller shrink of systems with smaller $m_{2}$, which is related to the minor amount of energy required to expel a less massive $\mathrm{CE}$. For the same reason, at given $m_{2}$, a less massive primary implies a smaller amount of shrinkage at the first CE. Therefore, for the CLOSE DD scheme

\footnotetext{
${ }^{6}$ Unlike for the WIDE $D D$ scheme, it seems adequate to restrict here to systems with $m_{1 \mathrm{R}} \geq m_{2 \mathrm{R}}$.
}

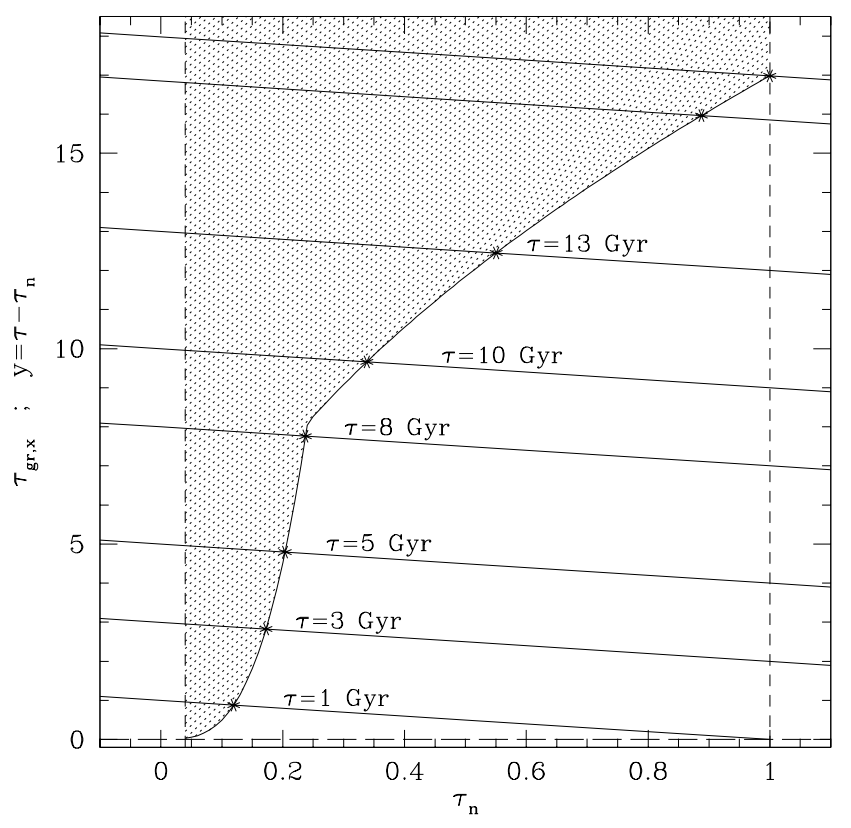

Fig. 7. Illustration of how the $\tau_{\mathrm{n}}^{\star}$ point, defining the branching in the CLOSE DD distribution of gravitational delays, shifts with increasing total delay $\tau$. The straight lines show the loci $y=\tau-\tau_{\mathrm{n}}$ for a few values of $\tau$; the curve shows the locus $\tau_{\mathrm{gw}, \mathrm{x}}\left(\tau_{\mathrm{n}}\right)$ : for illustrative purposes, the relation $\log \tau_{\mathrm{gw}, \mathrm{x}}=\min \left[-16.66+3.17 \log \tau_{\mathrm{n}} ; 5.4+0.52 \log \tau_{\mathrm{n}}\right]$ is used. Delay times are in Gyr. The asterisk marks the branching occurring at $\tau_{\mathrm{n}}=\tau_{\mathrm{n}}^{\star}$. The shaded region shows the domain over which $\mathrm{g}\left(\tau, \tau_{\mathrm{n}}\right)=1$. The dashed lines indicate the total range of nuclear delays, corresponding to the range of $m_{2}$ considered here, i.e. from 8 to $2 M_{\odot}$.

it seems appropriate to adopt a parametrization which emphasizes the systematic depletion of systems with long $\tau_{\mathrm{gw}}$ as $\tau_{\mathrm{n}}$ decreases, or the increase of the maximum gravitational delay in systems with smaller secondary mass. For simplicity, I consider directly $\tau_{\mathrm{gw}}$ as the independent variable, and assume that for each $m_{2}$ the differential distribution of the gravitational delays scales as $n\left(\tau_{\mathrm{gw}}\right) \propto \tau_{\mathrm{gw}}^{\beta_{\mathrm{g}}}$ between a minimum $\left(\tau_{\mathrm{gw}, \mathrm{i}}\right)$ and a maximum value $\left(\tau_{\mathrm{gw}, \mathrm{x}}\right)$. Admittedly, this choice is rather arbitrary; I just notice that, if the distribution of $\tau_{\mathrm{gw}}$ is mainly controlled by the distribution of $A$, one can write:

$n\left(\tau_{\mathrm{gw}}\right) \mathrm{d} \tau_{\mathrm{gw}} \propto n(A) \mathrm{d} A$

which, in combination with the relations $\tau_{\mathrm{gw}} \propto A^{4}$ and $n(A) \propto$ $A^{\beta_{\mathrm{a}}}$ becomes $n\left(\tau_{\mathrm{gw}}\right) \propto \tau_{\mathrm{gw}}^{\beta_{\mathrm{g}}}$ with $\beta_{\mathrm{g}}=-0.75+0.25 \beta_{\mathrm{a}}$.

With this assumption, the fraction of systems which manage to merge within $\left(\tau-\tau_{\mathrm{n}}\right)$ is:

$g\left(\tau, \tau_{\mathrm{n}}\right)= \begin{cases}0 & \text { for } \tau-\tau_{\mathrm{n}} \leq \tau_{\mathrm{gw}, \mathrm{i}} \\ \frac{\left(\tau-\tau_{\mathrm{n}}\right)^{1+\beta \beta_{\mathrm{g}}}-\tau_{\mathrm{gww}}^{1+\beta \beta_{\mathrm{g}}}}{\tau_{\mathrm{gw}, \mathrm{i}}^{+\beta \beta_{\mathrm{g}}}-\tau_{\mathrm{gw}, \mathrm{i}}^{1+\beta_{\mathrm{g}}}} & \text { for } \tau_{\mathrm{gw}, \mathrm{i}} \leq \tau-\tau_{\mathrm{n}} \leq \tau_{\mathrm{gw}, \mathrm{x}} \\ 1 & \text { for } \tau-\tau_{\mathrm{n}} \geq \tau_{\mathrm{gw}, \mathrm{x}}\left(\tau_{\mathrm{n}}\right)\end{cases}$

where $\tau_{\mathrm{gw}, \mathrm{x}}$ is an increasing function of $\tau_{\mathrm{n}}$. It is further assumed that the minimum gravitational delay $\tau_{\mathrm{gw}, \mathrm{i}}$ is independent of $m_{2}$ and small (compared to $\tau_{\mathrm{n}, \mathrm{i}}$ ), while the maximum gravitational delay is correlated to $\tau_{\mathrm{n}}$ via Eq. (30).

To better understand the behavior of the $g$ function, Fig. 7 illustrates how the second branching in Eq. (31) varies as $\tau_{\mathrm{n}}$ 
increases. The parallel straight lines show the $y=\tau-\tau_{\mathrm{n}}$ loci for selected values of $\tau$, ranging from 1 to $\sim 18 \mathrm{Gyr}$; the curve is one example for the $\tau_{\mathrm{gw}, \mathrm{x}}$ locus (different from Eq. (30) for illustrative purposes). The intercept between each straight line and the $\tau_{\mathrm{gw}, \mathrm{x}}$ curve (in $\tau_{\mathrm{n}}=\tau_{\mathrm{n}}^{\star}$ ) defines the branching of Eq. (31): at given $\tau$, systems with $\tau_{\mathrm{n}}$ shorter than $\tau_{\mathrm{n}}^{\star}$ have all merged, while only a fraction of the systems with $\tau_{\mathrm{n}}>\tau_{\mathrm{n}}^{\star}$ have already exploded ${ }^{7}$. Thus, over the shaded portion of the plane, $g\left(\tau, \tau_{\mathrm{n}}\right)=1$, i.e. the fraction of systems which, having a nuclear delay $\tau_{\mathrm{n}}$, merge within a total delay $\tau$ is constant and equal to 1 . Clearly, at long total delays, the area in the parameter space over which systems have already merged widens, and only those with long $\tau_{\mathrm{n}}$, and therefore long $\tau_{\mathrm{gw}}$, still give a variable contribution. When $\tau \geq \tau_{\mathrm{n}, \mathrm{x}}+\tau_{\mathrm{gw}, \mathrm{x}}\left(\tau_{\mathrm{n}, \mathrm{x}}\right), g\left(\tau, \tau_{\mathrm{n}}\right)=1$ for whatever $\tau_{\mathrm{n}}$, that is for all the SNIa precursors. Thus, in this scheme, the maximum delay time of the whole DD population from an instantaneous burst of SF is $\tau_{\mathrm{x}}=\tau_{\mathrm{n}, \mathrm{x}}+\tau_{\mathrm{gw}, \mathrm{x}}\left(\tau_{\mathrm{n}, \mathrm{x}}\right)$.

By varying the parameters of the close binary evolution (e.g. $\left.\alpha_{\mathrm{ce}}, f_{\mathrm{en}}\right)$ the $\tau_{\mathrm{gw}, \mathrm{x}}\left(\tau_{\mathrm{n}}\right)$ locus shifts. If evolution leads to systems which are all very close, the maximum gravitational delay will be small, and soon $\tau_{\mathrm{x}}$ will be reached, at which point the distribution function of the delay times for the CLOSE DDs drops to 0 .

\subsection{The distribution function of the delay times for DDs}

The distribution function of the delay times for the DDs is obtained by computing the derivative of Eq. (24):

$f_{\mathrm{Ia}}^{\mathrm{DD}}(\tau)=\frac{\mathrm{d}}{\mathrm{d} \tau} \int_{\tau_{\mathrm{n}, \mathrm{i}}}^{\min \left(\tau_{\mathrm{n}, \mathrm{x}}, \tau\right)} n\left(\tau_{\mathrm{n}}\right) g\left(\tau, \tau_{\mathrm{n}}\right) \mathrm{d} \tau_{\mathrm{n}}$

where $n\left(\tau_{\mathrm{n}}\right)$ is the distribution function of the nuclear delays of the SNIa progenitors, that is the $f_{\mathrm{Ia}}^{\mathrm{SD}}$ function derived in Sect. 3.2.

Equation (32) is solved by applying the Leibniz integral rule, as shown in Appendix A.4. The final function is:

$f_{\mathrm{Ia}}^{\mathrm{DD}}(\tau) \propto \int_{\tau_{\mathrm{n}, \mathrm{i}}}^{\min \left(\tau_{\mathrm{n}, \mathrm{x}}, \tau\right)} n\left(\tau_{\mathrm{n}}\right) S^{\mathrm{W}}\left(\tau, \tau_{\mathrm{n}}\right) \mathrm{d} \tau_{\mathrm{n}}$ for WIDE DDs

$f_{\mathrm{Ia}}^{\mathrm{DD}}(\tau) \propto \int_{\tau_{\mathrm{n}, \mathrm{nnf}}}^{\min \left(\tau_{\mathrm{n}, \mathrm{x}}, \tau\right)} n\left(\tau_{\mathrm{n}}\right) S^{\mathrm{C}}\left(\tau, \tau_{\mathrm{n}}\right) \mathrm{d} \tau_{\mathrm{n}}$ for CLOSE DDs

complemented with

$f_{\mathrm{Ia}}^{\mathrm{DD}}(\tau)=0 \quad$ for $\tau \leq \tau_{\mathrm{i}}$ and for $\tau \geq \tau_{\mathrm{x}}$

where

$S^{\mathrm{W}}\left(\tau, \tau_{\mathrm{n}}\right)= \begin{cases}f_{1,2}^{\mathrm{W}}\left(\tau-\tau_{\mathrm{n}}\right)^{-0.75+0.25 \beta_{\mathrm{a}}} & \text { for } \tau_{\mathrm{n}} \leq \tau-\tau_{\mathrm{gw}, \mathrm{i}} \\ 0 & \text { for } \tau_{\mathrm{n}} \geq \tau-\tau_{\mathrm{gw}, \mathrm{i}}\end{cases}$

$S^{\mathrm{C}}\left(\tau, \tau_{\mathrm{n}}\right)= \begin{cases}\frac{\left(\tau-\tau_{\mathrm{n}}\right)^{\beta \mathrm{g}}}{\tau_{\mathrm{gw}, \mathrm{x}}^{1+\beta_{\mathrm{g}}}-\tau_{\mathrm{gw}, \mathrm{i}}^{1+\beta \mathrm{g}}} & \text { for } \tau_{\mathrm{n}} \leq \tau-\tau_{\mathrm{gw}, \mathrm{i}} \\ 0 & \text { for } \tau_{\mathrm{n}} \geq \tau-\tau_{\mathrm{gw}, \mathrm{i}}\end{cases}$

${ }^{7}$ Notice that for $\tau \leq \tau_{\mathrm{n}, \mathrm{i}}+\tau_{\mathrm{gw}, \mathrm{x}}\left(\tau_{\mathrm{n}, \mathrm{i}}\right)$ there's no intersection between the two loci: in this range, the total delay considered is so short that $g\left(\tau, \tau_{\mathrm{n}}\right)<1$ even for the shortest $\tau_{\mathrm{n}}$. This regime is not visible in Fig. 7 because, for the chosen relation, $\tau_{\mathrm{n}, \mathrm{i}}+\tau_{\mathrm{gw}, \mathrm{x}}\left(\tau_{\mathrm{n}, \mathrm{i}}\right)=0.07 \mathrm{Gyr}$. $\tau_{\mathrm{n}, \mathrm{inf}}=\left\{\begin{array}{l}\tau_{\mathrm{n}, \mathrm{i}} \text { for } \tau<\tau_{\mathrm{n}, \mathrm{i}}+\tau_{\mathrm{gw}, \mathrm{x}}\left(\tau_{\mathrm{n}, \mathrm{i}}\right) \\ \tau_{\mathrm{n}}^{\star} \text { for } \tau \geq \tau_{\mathrm{n}, \mathrm{i}}+\tau_{\mathrm{gw}, \mathrm{x}}\left(\tau_{\mathrm{n}, \mathrm{i}}\right)\end{array}\right.$

and with the following meaning of the symbols:

- $\tau_{\mathrm{n}, \mathrm{i}}, \tau_{\mathrm{n}, \mathrm{x}}$ : respectively the nuclear timescales of the most and least massive secondary in the SNIa progenitors' binary systems; if $m_{2}$ ranges between 8 and $2 M_{\odot}, \tau_{\mathrm{n}, \mathrm{i}} \simeq 0.04 \mathrm{Gyr}$ and $\tau_{\mathrm{n}, \mathrm{x}} \simeq 1 \mathrm{Gyr}$

- $\tau_{\mathrm{gw}, \mathrm{i}}, \tau_{\mathrm{gw}, \mathrm{x}}$ : respectively the minimum and maximum gravitational delay of SNIa DD precursors, originated from systems with given $m_{2} ; \tau_{\mathrm{gw}, \mathrm{i}}$ is assumed independent of $m_{2}$; $\tau_{\mathrm{gw}, \mathrm{x}}$ is assumed (i) larger than the Hubble time for all SNIa precursors in the WIDE DD scheme, (ii) correlated with $\tau_{\mathrm{n}}$ as given by e.g. Eq. (30) in the CLOSE DD scheme;

$-\tau_{\mathrm{n}}^{\star}$ is the solution of the equation $\tau-\tau_{\mathrm{n}}=\tau_{\mathrm{gw}, \mathrm{x}}\left(\tau_{\mathrm{n}}\right)$, i.e. the minimum $\tau_{\mathrm{n}}$ which contributes to the explosions at epoch $\tau$, which increases with $\tau$ (see Fig. 7);

- $\tau_{\mathrm{i}}$ : the minimum total delay time $\left(\tau_{\mathrm{i}}=\tau_{\mathrm{n}, \mathrm{i}}+\tau_{\mathrm{gw}, \mathrm{i}}\right)$, assumed to be independent of $m_{2}$;

- $\tau_{\mathrm{x}}$ : the maximum total delay time, larger than the Hubble time for the WIDE $D D$; equal to the maximum delay of the least massive SNIa progenitor for the CLOSE DD: $\tau_{\mathrm{x}}=$ $\tau_{\mathrm{n}, \mathrm{x}}+\tau_{\mathrm{gw}, \mathrm{x}}\left(\tau_{\mathrm{n}, \mathrm{x}}\right)$

- $f_{1,2}^{\mathrm{W}}$ : the term describing the dependence on the mass of the DD systems, given by Eq. (29);

$-\beta_{\mathrm{a}}, \beta_{\mathrm{g}}$ : the exponents of the power law distributions adopted respectively for the final separations in the WIDE DD scheme, and for the gravitational delays in the CLOSE DD scheme; a flat distribution of $A$ corresponds to $\beta_{\mathrm{a}}=0$, and $\beta_{\mathrm{g}}$ in the vicinity of -0.75 (if $M_{\mathrm{DD}}$ varies in a small range).

In addition, the $(\alpha, \gamma)$ parameters need to be specified, in order to compute the distribution of the nuclear delays. In the models shown here, I have considered $\alpha=2.35$ and $\gamma=1$ : the dependence of $n\left(\tau_{\mathrm{n}}\right)$ on these parameters is modest for nuclear delays up to $\sim 1$ Gyr (see Fig. 3).

In both Eqs. (33) and (34) at each total delay $\tau$, the $f_{\mathrm{Ia}}^{\mathrm{DD}}$ function results from the sum of the contributions from systems with a range of $m_{2}$, and each contribution is proportional to a power of $\left(\tau-\tau_{\mathrm{n}}\right)$, the latter being just the gravitational delay of the progeny of systems born with secondary mass $m_{2}$, which end up in a SNIa event at epoch $\tau$.

The two equations correspond to different characterizations of SNIa precursors: Eq. (33) separately accounts for the sensitivity of the gravitational delay from the total mass of the DD systems (through the factor $f_{1,2}^{\mathrm{W}}$ ), and from the distribution function of the separations (through $\beta_{\mathrm{a}}$ ). At the same time, it assumes that at any $\tau$ up to the Hubble time, SNIa explosions come from all systems with $\tau_{\mathrm{n}} \leq \tau_{\mathrm{n}, \mathrm{x}}$. On the contrary, Eq. (34) emphasizes the systematics of the range of the gravitational delays as $m_{2}$ (and hence $\tau_{\mathrm{n}}$ ) varies: at each $\tau$, only systems with $\tau_{\mathrm{n}} \geq \tau_{\mathrm{n}}^{\star}$ contribute to the explosions, since those systems with shorter nuclear timescales have a too short maximum gravitational delay.

Neither of the two relations will strictly apply in nature, but they can be used to investigate on the general shape of the distribution function of the delay times in two extreme situations 
for what concerns the product of the Common Envelope evolution. As an illustration, Fig. 8 shows the distribution function of the delay times for the DD model for one specific choice of the parameters, as labeled. For comparison, the distribution function of the SD systems is also plotted. I recall here that $f_{\mathrm{Ia}}(\tau)$ is proportional to the SNIa rate following an instantaneous burst of SF.

Similar to the case of the SD model, the distribution function $f_{\mathrm{Ia}}^{\mathrm{DD}}(\tau)$ appears characterized by three regimes: first a rapid increase, followed by a slow decrease or a wide maximum, and finally a late epoch, pronounced decline. This shape can be viewed as a modification of the $f_{\mathrm{Ia}}^{\mathrm{SD}}$ function: for the DD model, early explosions are given by systems with short $\tau_{\mathrm{n}} A N D$ short $\tau_{\mathrm{gw}}$; the flat portion corresponds to those epochs at which the SNIa events come from many combinations of $\tau_{\mathrm{n}}$ and $\tau_{\mathrm{gw}}$; at late epochs we are left with systems with long $\tau_{\mathrm{gw}}$. Notice that when $\tau$ is large compared to $\tau_{\mathrm{n}, \mathrm{x}}$ Eq. (33) can be approximated as $f_{\mathrm{Ia}}^{\mathrm{DD}}(\tau) \propto \tau^{-0.75+0.25 \beta_{\mathrm{a}}}$ : compared to the SD model, this decline rate is considerably mild, and basically controlled by the dependence of the gravitational delay on the final separation $A$. A late epoch increase of $f_{\mathrm{Ia}}^{\mathrm{DD}}$ can be realized only if $\beta_{\mathrm{a}}$ is large and positive, corresponding to $n(A)$ distributions highly skewed toward large values of the final separations, which is very unlikely.

With respect to the WIDE DD, the CLOSE DD scheme yields a distribution of the delay times which is steeper both at the intermediate and at the late epochs. This reflects the relative paucity of systems with long $\tau_{\mathrm{gw}}$ in this scheme of evolution. However, in spite of the very different assumptions, the overall behavior of the $f_{\text {Ia }}$ functions in Fig. 8 look similar. In Sect. 5, the difference between the models will be better quantified, by considering the distribution function of the delay times suitably normalized.

For the DD model, at $\tau=1$ Gyr there is a cusp: mathematically this is due to the discontinuity of the $S^{\mathrm{W}}$ and $S^{\mathrm{C}}$ functions, coupled with the upper limit of integration for $f_{\mathrm{Ia}}^{\mathrm{DD}}$. In practice, the cusp occurs at the epoch at which the systems with smallest $m_{2}$ start contributing to the SNIa rate, that is $\tau=\tau_{\mathrm{n}, \mathrm{x}}+$ $\tau_{\mathrm{gw}, \mathrm{i}}$. After this epoch, increasing $\tau$ corresponds to include systems with longer $\tau_{\mathrm{gw}}$ but NOT longer $\tau_{\mathrm{n}}$. The prominence of the cusp is related to the $\beta_{\mathrm{a}},\left(\beta_{\mathrm{g}}\right)$ exponents which control the $n\left(\tau_{\mathrm{gw}}\right)$ distribution toward the minimum $\tau_{\mathrm{gw}, \mathrm{i}}$.

Finally, the different scheme used to compute the distribution function of $m_{2}$, i.e. whether using Eqs. (16) or (20) has a very modest impact on the final $f_{\mathrm{Ia}}^{\mathrm{DD}}(\tau)$ function. As for the SD model, the use of the Greggio \& Renzini (1983) scheme yields a relatively larger rate at late epochs.

\subsection{Dependence on key parameters}

I turn now to consider the dependence of the function $f_{\mathrm{Ia}}^{\mathrm{DD}}$ on the several parameters that need to be specified.

Both the upper and the lower limits to the mass of the secondary component in the SNIa progenitor systems are subject to some uncertainty, which reflects on the parameters $\tau_{\mathrm{n}, \mathrm{i}}$ and $\tau_{\mathrm{n}, \mathrm{x}}$. The most massive CO DD systems come from progenitors in which both components are $\sim 8 M_{\odot}$ stars; if these systems manage to produce a SNIa event, $\tau_{\mathrm{n}, \mathrm{i}}$ is about $0.04 \mathrm{Gyr}$. As mentioned in the introduction, though, the ultimate fate of a double CO WD might be an accretion induced collapse, rather than a central carbon deflagration, depending on the modalities of accretion (e.g. the accretion rate and the angular momentum deposition on the WD). This question is highly debated in the current literature (e.g. Piersanti et al. 2003; and Saio \& Nomoto 2004), to the aim of establishing the likelihood of the DD channel as SNIa precursors in general. However, notice that if the occurrence of the accretion induced collapse depends on the mass of the DD components, the DD channel remains a valid SNIa progenitor, but $\tau_{\mathrm{n}, \mathrm{i}}$ changes to become the nuclear lifetime of the most massive secondary in a system which avoids the accretion induced collapse. Since in the current literature there's no claim of this effect, this possibility is neglected here, and all the models adopt $\tau_{\mathrm{n}, \mathrm{i}}=0.04 \mathrm{Gyr}$. As already mentioned, most double CO WDs come from systems with $m_{2}$ greater than $2 M_{\odot}$, so that a reasonable value for $\tau_{\mathrm{n}, \mathrm{x}}$ is $1 \mathrm{Gyr}$. However, the likelihood of a successful explosions may well be decreasing as $m_{2}$ approaches this limit, due to the requirement that $M_{\mathrm{DD}}$ exceeds the Chandrasekhar mass. Therefore, $\tau_{\mathrm{n}, \mathrm{x}}$ is treated as a parameter, and I show here the results obtained with $\tau_{\mathrm{n}, \mathrm{x}}=0.4$, 0.6 and $1 \mathrm{Gyr}$, corresponding to a lower limit to $m_{2}$ in SNIa progenitors of $\sim 3,2.5$ and $2 M_{\odot}$, respectively.

Given the high degree of shrinkage which is obtained when applying the standard CE recipe, the lower limit to the gravitational delay $\left(\tau_{\mathrm{gw}, \mathrm{i}}\right)$ is likely to be very small. For this reason I adopt here a nominal value of $\tau_{\mathrm{gw}, \mathrm{i}}=0.001 \mathrm{Gyr}$ in most computations. However, the minimum gravitational delay could be larger, especially for systems with high initial mass ratio, if e.g. Nelemans et al. (2001) scheme of evolution applies. Thus, I explore the sensitivity of the results on $\tau_{\mathrm{gw}, \mathrm{i}}$ only for the WIDE DD scheme, adopting a very large value of $\tau_{\mathrm{gw}, \mathrm{i}}=$ 0.1 Gyr. For the CLOSE DD scheme the critical gravitational timescale is instead $\tau_{\mathrm{gw}, \mathrm{x}}$ : e.g. low values of $\alpha_{\mathrm{ce}}$ produce a maximum gravitational delay shorter than the Hubble time even for the lowest mass systems (see Fig. 6). Two options for the relation $\tau_{\mathrm{gw}, \mathrm{x}}\left(\tau_{\mathrm{n}}\right)$ are considered.

Without specific population synthesis computations, little can be said about the $\beta_{\mathrm{a}}$ and the $\beta_{\mathrm{g}}$ parameters. Since the distribution of the separations in primordial binaries is typically taken to scale as $n\left(A_{0}\right) \propto A_{0}^{-1}$ (e.g. Iben \& Tutukov 1984; Han 1998; Nelemans et al. 2001), I consider the three values $\beta_{\mathrm{a}}=-0.9,0,+0.9$ which correspond to assuming that, as a result of evolution, the distribution of the separations (i) remains basically unchanged; (ii) flattens off, so that any value of $A$ is equally probable; (iii) changes slope, so that more DD systems are found with large $A$. The values of the $\beta_{\mathrm{g}}$ parameter explored here are related to the three $\beta_{\mathrm{a}}$ values via $\beta_{\mathrm{g}}=-0.75+0.25 \beta_{\mathrm{a}}$ (see Sect. 4.3.2), and are $\beta_{\mathrm{g}}=-0.975,0.75,-0.525$. Notice that, since most combinations of $\left(M_{\mathrm{DD}}, A\right)$ lead to short $\tau_{\mathrm{gw}}$, positive values for $\beta_{\mathrm{g}}$ or even a flat distribution of the gravitational delays are extremely unlikely.

Figure 9 illustrates the dependence of $f_{\mathrm{Ia}}^{\mathrm{DD}}(\tau)$ on the parameters related to the timescales (left panels), and on those related to the distribution of the separations (right panels). The left panels show how the late epoch decline starts at $\tau=\tau_{\mathrm{n}, \mathrm{x}}+$ $\tau_{\mathrm{gw}, \mathrm{i}}$, as argued in the previous section. The more massive is the 


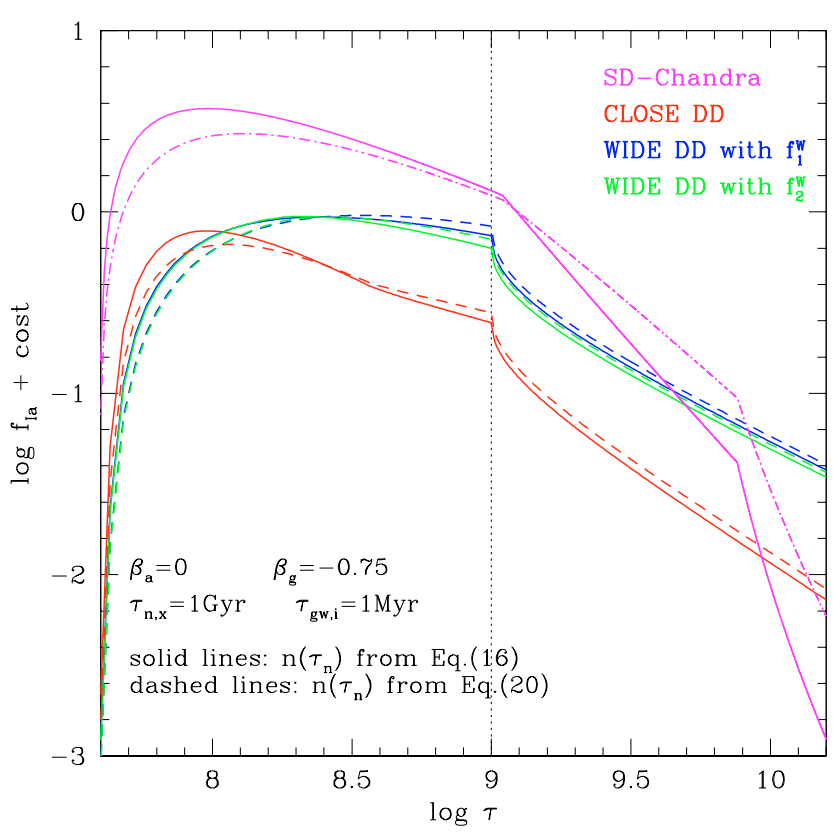

Fig. 8. Illustration of the distribution function of the delay times in the DD model for the labeled values of the parameters. The red lines show the result for the CLOSE DD scheme; the blue and green for the WIDE $D D$ scheme, respectively when $M_{\mathrm{DD}}$ is strongly and mildly correlated with $m_{2}$. Equation (30) has been used for $\tau_{\mathrm{gw}, \mathrm{x}}\left(\tau_{\mathrm{n}}\right)$. For comparison, the magenta curves show the distribution function of the delay times in the SD model, for Chandrasekhar explosions. In all models the distribution of the nuclear delays is derived with $\alpha=2.35$ and $\gamma=1$.

lower limit to $m_{2}$ of the SNIa progenitors', the larger is the fraction of early explosions, i.e. the shorter will be the timescale for the release of the bulk of the nucleosynthetic products to the interstellar medium. If SNIas come mostly from DDs which are born with relatively wide separations, such that $\tau_{\mathrm{gw}, \mathrm{i}}$ is large, the distribution function of the delay times behaves like the magenta curve in the lower left panel: the first explosion occurs at $\tau=\tau_{\mathrm{n}, \mathrm{i}}+\tau_{\mathrm{gw}, \mathrm{i}}$, after which the rate increases rapidly up to the start of the late epoch decline. The magenta curve in the top left panel shows instead what happens if the close binary evolution produces a large degree of shrinkage: the $f_{\mathrm{Ia}}^{\mathrm{DD}}$ function is more skewed toward short delays, and it does not provide systems with delays longer than $\tau_{\mathrm{n}, \mathrm{x}}+\tau_{\mathrm{gw}, \mathrm{x}}\left(\tau_{\mathrm{n}, \mathrm{x}}\right)$. This illustrates the potential difficulty of accounting for SNIa in Elliptical galaxies, if the close binary evolution produces too close DDs.

The right panels in Fig. 9 show instead how the distribution function of the delay times depends on $\beta_{\mathrm{a}}$ and on $\beta_{\mathrm{g}}$ in the special case of $\tau_{\mathrm{n}, \mathrm{x}}=0.4 \mathrm{Gyr}$ and $\tau_{\mathrm{gw}, \mathrm{i}}=1 \mathrm{Myr}$. In general, $f_{\mathrm{Ia}}^{\mathrm{DD}}$ appears to be fairly sensitive to these exponents, with more early explosions as the distribution function of the separations of the DDs is more populated at the low $A$ end.

\section{Discussion}

Very schematically, the distribution function of the delay times of SNIa progenitors derived in the previous sections

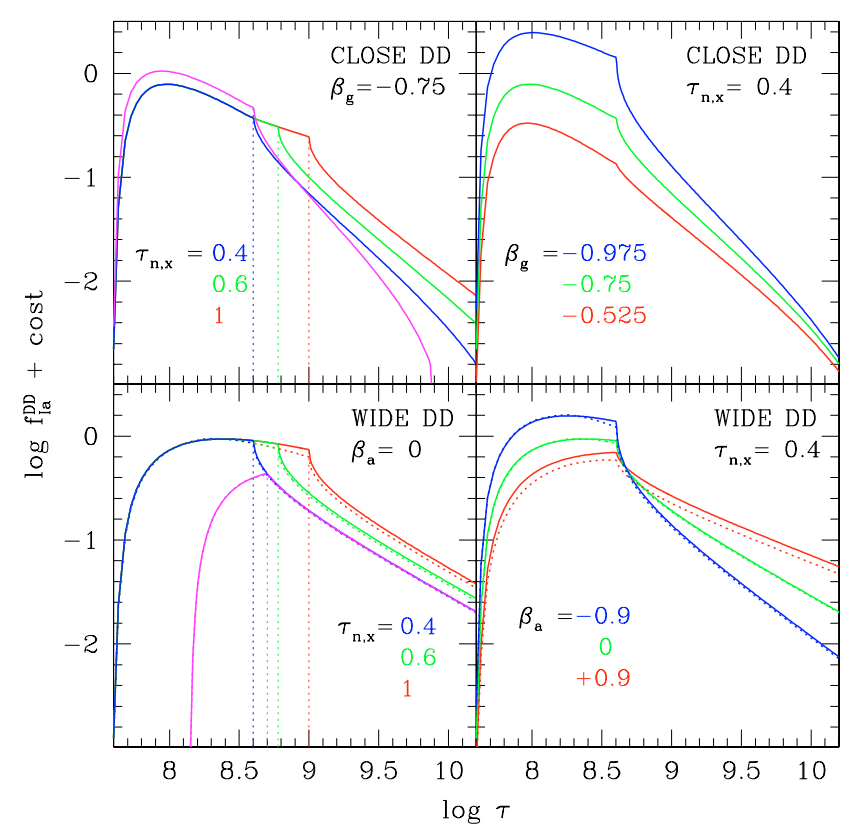

Fig. 9. Sensitivity of the distribution function of the delay times for DD progenitors on various parameters. The top panels refer to the CLOSE DD scheme, the bottom panels to the WIDE DD scheme of evolution. The left panels show the dependence on the $\tau_{\mathrm{n}, \mathrm{x}}$ parameter, with the color encoding as labeled. In addition, the magenta line in the top panel has been obtained with $\tau_{\mathrm{gw}, \mathrm{x}}\left(\tau_{\mathrm{n}}\right)$ as given by Eq. (30) decreased by 0.6 Dex, mimicking the effect of lower $\alpha_{\text {ce }}$. The magenta line in the bottom panel shows instead the effect of adopting $\tau_{\mathrm{gw}, \mathrm{i}}=$ $0.1 \mathrm{Gyr}$ in the WIDE DD scheme. In all other models $\tau_{\mathrm{gw}, \mathrm{i}}=1 \mathrm{Myr}$ is used. The right panels show the effect of varying $\beta_{\mathrm{a}}$ and $\beta_{\mathrm{g}}$. The dotted lines in the bottom panels show the results of using $f_{1,2}^{\mathrm{W}}=f_{2}^{\mathrm{W}}$ in the computation of $f_{\mathrm{Ia}}^{\mathrm{DDW}}$. All curves have been computed with $n\left(\tau_{\mathrm{n}}\right)$ derived from Eq. (16) with $(\alpha, \gamma)=(2.35,1)$.

for both Single and Double Degenerate models is characterized by:

- an early steep rise;

- an intermediate phase, hereafter referred to as the wide maximum;

- a decline phase.

The minimum and maximum delay times, the duration of the intermediate phase, and the slopes of the intermediate and late phases are different for the Single and the Double Degenerate progenitors, and are controlled by a few key parameters.

For the SD progenitors, the minimum delay time is equal to the MS lifetime of the most massive secondary in the primordial binary producing a SNIa event; the wide maximum phase lasts until a delay time equal to the MS lifetime of the least massive primary evolving into a CO WD suitable as SNIa precursor; the decline phase becomes very steep at late epochs, if the requirement of building up to the Chandrasekhar mass limits the SNIa progenitors to systems with more massive primaries in combination with less massive secondaries. In the decline phase, the slope of the $f_{\text {Ia }}$ function depends on the IMF, and on the distribution of the mass ratios. In particular, the flatter the distribution of the mass ratios, the larger the fraction of systems with long delays, at fixed IMF slope. For the Chandrasekhar 
exploders, the maximum delay is equal to the MS lifetime of the secondary whose envelope is massive enough to ensure that the most massive CO WD reaches the Chandrasekhar limit upon accretion. For the Sub-Chandra exploders, the maximum delay is equal to the MS lifetime of the secondary with a massive enough envelope to provide the minimum layer for helium ignition on top of the companion. Both these constraints depend upon the efficiency of the accretion process.

For the DD progenitors, the minimum delay time is equal to the MS lifetime of the most massive secondary in a SNIa progenitor system, plus the minimum gravitational delay; the wide maximum phase lasts up to a delay equal to the MS lifetime of the least massive secondary in a SNIa progenitor system, again plus the minimum gravitational delay. The slope of the decline phase is sensitive to the distribution function of the separation of the DD systems at birth. In addition, the overall distribution function of the delay times is steeper if a correlation exists such that the more massive binaries merge on a shorter timescale than the less massive ones, due to a more pronounced shrinking of the system. This happens in the standard treatment of the CE evolution (CLOSE DD scheme). Finally, the maximum delay time for the DDs is basically equal to the gravitational delay of the least massive and widest DD progenitor: if the CE stages were to induce a high degree of shrinking, the maximum delay could well be shorter than the Hubble time.

\subsection{Comparison with the results of populations synthesis codes}

These $f_{\text {Ia }}$ functions have been derived with the aim of providing a general characterization of the distribution function of the delay times for the various potential SNIa progenitors, and a number of convenient, though astrophysically motivated, approximations have been introduced. It is thus very important to compare the general shape of these functions to the results of the population synthesis codes, which follow the individual evolution of close binaries in detail. Unfortunately, the distribution of the delay times of the SNIa events, or equivalently the SNIa rate following an instantaneous burst of Star Formation (see Eq. (5)), is not commonly found in the literature. Most authors rather quote the current SNIa rate in the Galaxy, which, following Eq. (8), gives information on the total realization probability of the Ia channel (i.e. $A_{\text {Ia }}$ ), but not on the shape of the $f_{\text {Ia }}$ function. The most suitable paper to perform a detailed comparison between the analytic functions presented here and the results of a population synthesis code is the one by YL.

Figure 2 in YL shows the SNIa rate following an instantaneous burst of Star Formation. Four types of precursors appear in this figure: the DD-Ch, i.e. Chandrasekhar Double Degenerate exploders; the SG-Ch, produced by the evolutionary path of the SD-Chandra considered here; the He-ELD and SG-ELD, which are two flavors of the Sub-Chandra Single Degenerate channel, the difference being that the former come from systems with $m_{2}$ greater than $2.5 M_{\odot}$, which donate helium to the degenerate companion, while the latter are systems in which $m_{2}$ is smaller than $2.5 M_{\odot}^{8}$, which donate $\mathrm{H}$,

\footnotetext{
${ }^{8}$ To be precise, YL quote a secondary mass below (2-3 $\left.M_{\odot}\right)$.
}

converted to helium of top of the CO WD. The He-ELD and SG-ELD can be viewed as two complementary paths building up into the broad Sub-Chandra category considered here.

The numerical simulations follow the evolution of one single population of binaries, which evolve through mutually exclusive channels; the $f_{\text {Ia }}$ functions presented here, instead, are thought of as alternative to each other, for the total stellar population. One could consider a scenario in which SNIa come from different channels, and construct a composite analytic distribution function of the delay times by properly assigning the various key parameters, and the realization probabilities of each channel. I prefer to avoid this approach and perform the comparison between the results presented here and YL's by taking into account the different mass ranges which evolve into the different channels.

Reading off Fig. 2 in YL, the rate for the DD-Ch exploders starts at a delay time of $\log t \simeq 7.4$, reaches a maximum shortly before $0.1 \mathrm{Gyr}$, and then drops; for delays in excess of about $0.3 \mathrm{Gyr}$ the trend is close to a power law with a slope of $\sim-1.2$. At $10 \mathrm{Gyr}$, the rate has dropped of 2.2 Dex with respect to its value at maximum. YL state that in their simulations, the SNIas typically come from binaries with primary components in the range between 4 and $10 M_{\odot}$, and that the DD-Ch channel applies to the systems with secondaries more massive than $4 M_{\odot}$. The upper limit on $m_{1}$ is larger than the $8 M_{\odot}$ adopted here because the evolution in a close binary can prevent $\mathrm{C}$ ignition before the loss of the envelope in stars less massive than $\sim 10 M_{\odot}$ (Iben \& Tutukov 1984). The evolutionary lifetime of a $10 M_{\odot}$ star (with solar metallicity) is about $25 \mathrm{Myr}$, which is also the delay time at which the first DD-Ch events appear to occur; the lifetime of a $4 M_{\odot}$ star is about $0.18 \mathrm{Gyr}$, close to the duration of the peak in the YL DD-Ch curve. Since YL adopt a description of the evolution during both CE phases similar to Eq. (26), the analogue of their DD-Ch case would be a CLOSE DD model with $\tau_{\mathrm{i}}=0.025 \mathrm{Gyr}$ and $\tau_{\mathrm{n}, \mathrm{x}}=0.18 \mathrm{Gyr}$, while nothing can be said about the adequate value of the $\beta_{\mathrm{g}}$ parameter. Using Eq. (30) with such short $\tau_{\mathrm{n}, \mathrm{x}}$, the maximum gravitational delay is much shorter than the Hubble time; on the other hand, as illustrated in Fig. $6, \tau_{\mathrm{gw}, \mathrm{x}}$ is very sensitive to the various parameters used to describe the CE evolution. In order to compare the analytic function for the DD model to YL results I consider a relation for $\tau_{\mathrm{gw}, \mathrm{x}}$ obtained from Eq. (30) plus a zero point shift of -0.6 , so as to recover maximum delays exceeding the Hubble time for the less massive SNIa progenitors. This case is shown in Fig. 10 for three values of the $\beta_{\mathrm{g}}$ parameter. It can be seen that the analytic $f_{\mathrm{Ia}}$ functions are very similar to the DD-Ch curves in YL; in particular, the case with $\beta_{\mathrm{g}}=-0.75$ is very well approximated by a power law with a slope of -1.27 for $\tau \gtrsim 0.5 \mathrm{Gyr}$, and at $10 \mathrm{Gyr}$ its $f_{\mathrm{Ia}}$ is $2.7 \mathrm{Dex}$ lower than its maximum value. This is a remarkable similarity, given the completely different ways in which the two functions have been obtained. Notice that in the range $8.7<\log t<9.5$ the match is better than this, since the analytic curves steepen when approaching the maximum delay time.

It is worth to point out that the $f_{\text {Ia }}$ functions for the CLOSE DD scenario are also in broad agreement with the SNIa rate following an instantaneous burst of star formation 


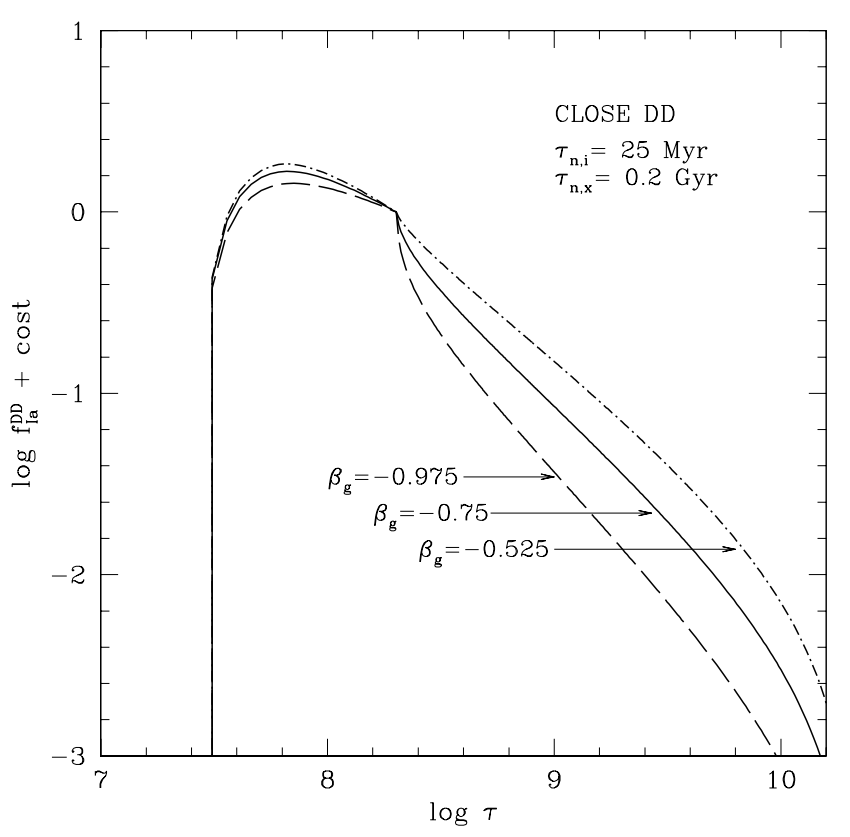

Fig. 10. Distribution function of the delay times for DD progenitors for a choice of parameters which represent the DD-Ch evolutionary channel in YL. See text for more details.

for other renditions of the DD scenario (Tutukov \& Yungelson 1994; Ruiz-Lapuente \& Canal 1998).

The similarity between the analytic and numerical functions for the SD, Sub-Chandra cases is much less apparent; still the differences can be understood in terms of different mass ranges populating this channel. After a steep rise, starting at $\log t=7.5$ (close to the MS lifetime of an $8 M_{\odot}$ star), the rate from the He-ELD channel has a wide maximum, followed by a rather abrupt drop, which sets in at about $0.5 \mathrm{Gyr}$. The latter is the evolutionary lifetime of a $\sim 2.7 M_{\odot}$ star, not far from the least massive secondary populating this channel, i.e. 2.5 $M_{\odot}$. Systems with lower secondary mass do evolve on a longer timescale, but do not go through the He-ELD channel. On the other hand, the rate from the SG-ELD exploders starts at a delay time of $0.6 \mathrm{Gyr}$, in correspondence to the lifetime of the most massive secondary which evolves through this channel. The rate rapidly reaches a maximum and then starts declining with a trend close to a power law with a slope of -1.6 . At a delay time of $\sim 5.6 \mathrm{Gyr}$ the rate drops dramatically. For comparison, the slope after the wide maximum of the blue, dot-dashed $f_{\mathrm{Ia}}^{\mathrm{SD}}$ curve in Fig. 3 is $\simeq-1.7$. It is tantalizing to conclude that the analytic formulation presented here for the Sub-Chandra exploders reproduces the general trend of the He-ELD + SG-ELD channels in YL, with the late dramatic drop possibly related to inefficient accretion from low mass secondaries.

Finally, the rate for the SG-Ch channel is indeed very different from the analogue analytic $f_{\mathrm{Ia}}^{\mathrm{SD}}$ functions in Fig. 3. However, as stated by YL, only systems with $m_{2}$ lower than $2.5 M_{\odot}$ evolve along this path, the more massive secondaries becoming either a DD-Ch, or a He-ELD. Therefore, the late start of the rate for this channel is understood in terms of a late $\tau_{\mathrm{n}, \mathrm{i}}$; the rapid drop at delays in excess of $1 \mathrm{Gyr}$ could instead reflect a low accretion efficiency. Actually, the comparison of the predictions for the Single Degenerate Chandrasekhar channel is particularly difficult because of the different approaches, as anticipated above: the systems evolving through the SG-Ch channel are clearly a minority in the population synthesis code.

The general conclusion is that the analytic distribution function of the delay times presented here can provide an excellent match to the results of the population synthesis codes, once the appropriate mass ranges and evolutionary timescales are assumed for the individual evolutionary paths. Obviously, the numerical simulations also estimate the realization probability of the various channels, and the total realization probability of the Ia event, that is the $A_{\text {Ia }}$ factor. There's no attempt here at evaluating this factor, which can either be taken from observational estimates, following Eq. (8), or from the population synthesis results.

The analytic approach offers several advantages, most notably:

(i) an easy way to explore the consequences on the evolution of stellar systems of the various candidates, viewed as alternative SNIa progenitors;

(ii) it has a built in parametrization of the key properties of the alternative candidates, i.e. mass ranges, IMF and distribution of the mass ratios, distribution of the separations of the DD systems;

(iii) a flexible tool to build up an overall distribution of the delay times by mixing the different analytic $f_{\text {Ia }}$ functions, weighted by the relative contribution of the individual channels to the total realization probability, as astrophysical considerations may suggest.

\subsection{Comparison between different SNla candidates}

For a meaningful comparison of the distribution functions of the delay times from the various potential SNIa progenitors it is necessary to normalize the $f_{\text {Ia }}$ functions. Among the various possibilities, a convenient normalization is to consider $\int_{\tau_{\mathrm{x}}}^{\tau_{\mathrm{x}}} f_{\mathrm{Ia}}(\tau) \cdot \mathrm{d} \tau=1$ (see Sect. 2): in this way, the specific SNIa rate at the current epoch in a system which formed its stars in an initial star formation episode of duration $\Delta t$ is given by (see Eq. (10)):

$$
\frac{\dot{n}_{\mathrm{Ia}}^{E}(t)}{\mathcal{M}}=k_{\alpha} \cdot A_{\mathrm{Ia}} \cdot\left\langle f_{\mathrm{Ia}}\right\rangle_{t-\Delta t, t}
$$

where $\left\langle f_{\mathrm{Ia}}\right\rangle_{t-\Delta t, t}$ is the average of the normalized distribution function of the delay times over the age range of the stars in the system, and $A_{\text {Ia }}$ is the realization probability of the SNIa scenario. I recall here that $k_{\alpha}$ depends on the IMF, and is equal to $1.55,2.83$ respectively for Kroupa and Salpeter IMFs. Figure 11 shows some selected cases of the analytic distribution functions normalized to 1 in the range between 0 and 12 Gyr. Although the maximum delay for the different candidates plotted here is larger than $12 \mathrm{Gyr}$, most distributions steepen enough at late times that the fraction of SNIa explosions at delay times exceeding $12 \mathrm{Gyr}$ is negligible. In the 
attempt of showing a wide range of possible solutions, the plotted cases adopt the following parameters:

(i) for the SD models, the solid lines have been computed with $\gamma=1$, the dashed lines with $\gamma=0.005$; the latter choice enhances the relative number of low mass secondaries (see Fig. 2);

(ii) for the DD models, the solid lines have been computed with $\beta_{\mathrm{a}}=0, \beta_{\mathrm{g}}=-0.75$; the dashed lines with $\beta_{\mathrm{a}}=+0.9$, $\beta_{\mathrm{g}}=-0.975$ for the WIDE DDs and the CLOSE DDs respectively. The latter cases are meant to illustrate the wide range of decline rates allowed by these options.

For a given realization probability of the SNIa event $\left(A_{\text {Ia }}\right)$ all models plotted in Fig. 11 provide the same total number of SNIa within 12 Gyr from a burst of SF of given total mass, but the events are differently distributed in time. Although for all of the models most of the events occur within the first $1 \mathrm{Gyr}$, in the WIDE DD scheme the explosions are more evenly spread over the whole 12 Gyr range. Both the CLOSE DDs and the SD Sub-Chandra model yield an age distribution of the events very skewed at the early epochs; the SD-Chandra model exhibits a dramatic drop at late epochs, related to the requirement of building up to the Chandrasekhar mass by accreting the envelope of low mass secondaries.

The models in Fig. 11 can be compared to the observed SNIa rate per unit mass in elliptical galaxies measured by Mannucci et al. (2005, hereafter M2005), which is quoted of $0.044(+0.016)(-0.014) \mathrm{SNuM}$, or $0.044(+0.016)(-0.014) \times$ $10^{-3}$ events per $M_{\odot}$ per Gyr. If $10 \%$ of the stars in the mass range from 3 to $8 M_{\odot}$ end up as SNIa, the factor $\left(k_{\alpha} A_{\mathrm{Ia}}\right)$ is about $(2,3) \times 10^{-3}$ respectively for Salpeter and Kroupa IMF. Adopting $k_{\alpha} A_{\mathrm{Ia}}=2.5 \times 10^{-3}$, and inserting $\left(\dot{n}_{\mathrm{Ia}}^{E}(t) / \mathcal{M}\right)=$ $0.044 \times 10^{-3}$, Eq. (39) yields $\left\langle f_{\mathrm{Ia}}\right\rangle_{t-\Delta t, t}=0.0176 \mathrm{Gyr}^{-1}$, which is the level indicated by the black line in Fig. 11. The dashed region shows the upper and lower limit relative to the range of the SNIa rate quoted by M2005 for E/S0 galaxies. For a larger realization probability of the SNIa event, following either from a wider range of progenitor masses, or from a larger probability of the SNIa channel within a given mass range, the observational constraint shifts downward.

The intercept between the theoretical $f_{\text {Ia }}$ functions and the observational constraint yields the average age which the stars in early type galaxies should have in order to reproduce the data. So, e.g. the SD-Chandra model is able to fit the data if either the stellar population in Es is young, or if the realization probability of the SNIa scenario is larger than what adopted. Both the age of the stars in Es and the $k_{\alpha} A_{\text {Ia }}$ factors are uncertain; therefore Fig. 11 does not allow us to draw stringent conclusions about the best model for SNIa precursors. However, the figure shows the interplay between the various quantities.

At 12 Gyr the SD models with $\gamma=1$ fall short by about one order of magnitude with respect to the level indicated by the observations. The mismatch is more severe for the Chandra case. Such a big discrepancy is difficult to recover either by increasing the realization probability of the SNIa scenario, or by invoking a younger age for Ellipticals: on the one hand the black level in Fig. 11 already assumes that an important fraction of stars (i.e. $\simeq 10 \%$ ) in the suitable mass range end up as
SNIa. On the other hand, M2005 data refers to a sample of more than 2000 early type objects, and the spectrophotometric properties of this class of galaxies strongly suggest that they are old (see e.g. Renzini 1999; Peebles 2002). The only possibility to reconcile the SD model with the SNIa rate in ellipticals seems to be that of assuming a very low $\gamma$ (dashed curves), so that the distribution of the secondaries is maximally populated at the low mass end. Even so, an accretion efficiency close to $100 \%$ is required for the SD-Chandra models to meet the observations, which seems unlikely, as argued in Sect. 3.2.

The DD models more easily account for the observations, provided that gravitational delays as long as the Hubble time are realized, i.e. the common envelope phases do not lead to a too severe shrinking of the DD systems. The steepest $f_{\text {Ia }}$ function (CLOSE DD with $\tau_{\mathrm{n}, \mathrm{x}}=0.4 \mathrm{Gyr}$ and $\beta_{\mathrm{g}}=-0.975$ ) fall short by a factor of $\sim 5$ with respect to the observational limit, and are thus unfavored. Notice that the mild slope of the $f_{\mathrm{Ia}}$ function from intermediate ages onward, implies that assuming a younger age for Ellipticals does not efficiently improve the fit for this kind of models. The illustration clearly shows that lower $A_{\text {Ia }}$ and/or older ages for ellipticals are accommodated with WIDE DD models.

The normalization chosen for Fig. 11 corresponds to assuming that all models, with the same realization probability, yield the same total number of SNIa out of a stellar generation of unit mass (and therefore the same chemical enrichment). In a different approach, Fig. 12 shows the models normalized to their value at $10 \mathrm{Gyr}$ : this corresponds to forcing all of them to fit the current SNIa rate in Es with an average stellar age of $10 \mathrm{Gyr}$, albeit with different values for the factor $k_{\alpha} A_{\text {Ia }}$. The realization probabilities required by this normalization are of the order of $10^{-2}$ for the SD models, of $10^{-3}$ for the DD models, but there is a noticeable dependence of the $A_{\text {Ia }}$ factors on the various parameters defining the models, including the formalism to describe the binary population (i.e. whether Eqs. (16) or (20) are used). Only the SD-Chandra models with low $\epsilon$ do require a totally unrealistic realization probability, corresponding to $\gtrsim 100 \%$ of the stars with mass between 2 and $8 M_{\odot}$, and will not be considered further. Once normalized in this way, the various models correspond to dramatically different evolution over cosmic time of the SNIa rate from a burst of SF (notice that the rate is plotted on a logarithmic scale). This property offers an important tool to discriminate among the models by looking at the impact on the large scales, like the Iron Mass-to-Light ratio in Clusters of galaxies, the evolution with redshift of the SNIa rate in Ellipticals, and the systematic trend of the SNIa rate with galaxy type (Greggio 2005). The latter point is addressed in the following section.

To conclude this section, Fig. 13 shows the cumulative number of SNIa explosions as a function of age for an instantaneous burst of SF, normalized to the total number of events within 12 Gyr. This figure illustrates another distinctive characteristic of the various SNIa models: the time scale over which $50 \%$ of the total SNIa explosions from an instantaneous burst of SF have occurred. This timescale can be taken as indicative of the typical delay with which the Fe from SNIa is released to the Interstellar Medium, thereby decreasing its $\alpha / \mathrm{Fe}$ ratio. Within the explored range of models such timescales varies 


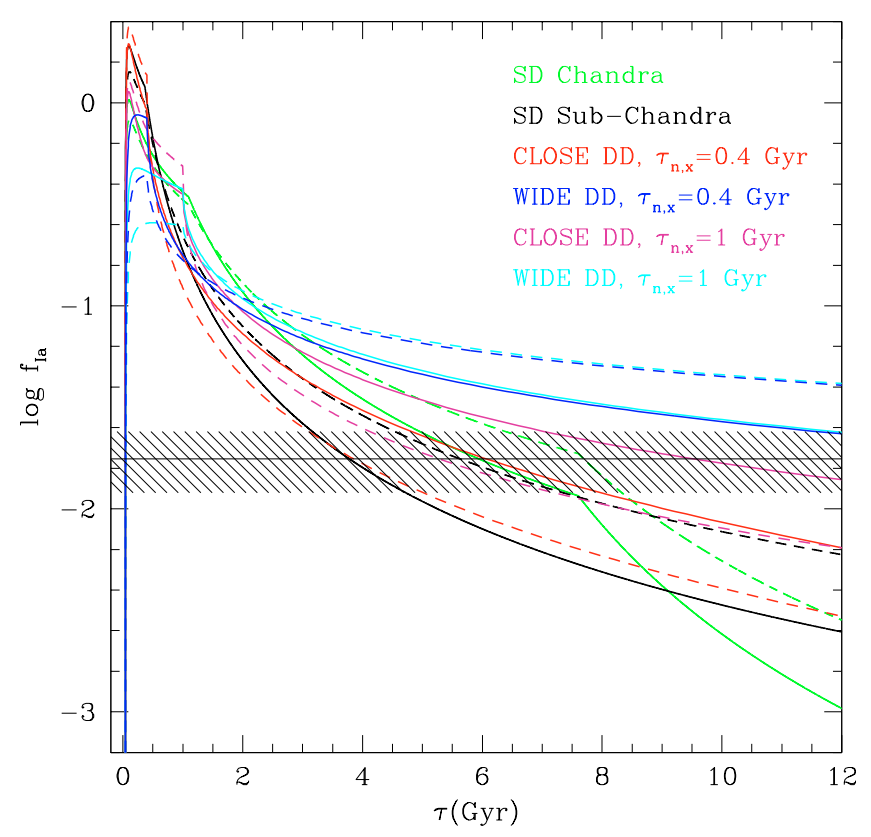

Fig. 11. Distribution function of the delay times for SD and for DD progenitors as labeled. The plotted functions have been normalized to 1 in the range $\tau \leq 12 \mathrm{Gyr}$, and the normalization constant is in units of $\mathrm{Gyr}^{-1}$. For the all models the distribution function of the secondaries has been derived using Eq. (16) with $\alpha=2.35$. See text for more details.

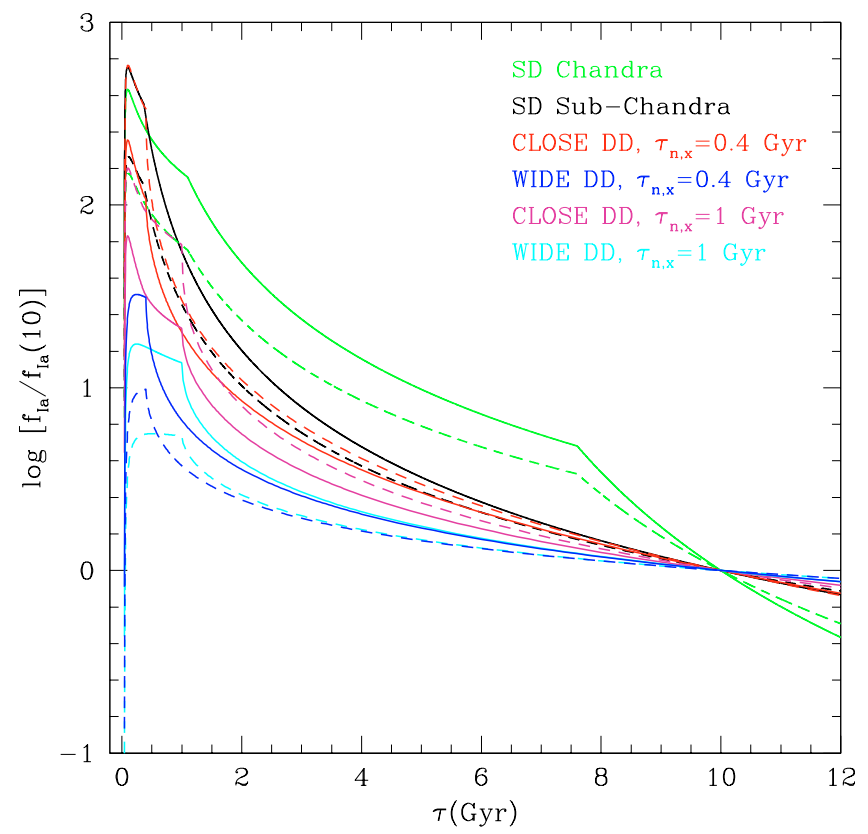

Fig. 12. Distribution functions of the delay times for the same models shown in Fig. 11 with the same color and line coding. The functions are here normalized to give the same value at $10 \mathrm{Gyr}$ in order to illustrate the different early epoch behavior of models which reproduce the current SNIa rate in Ellipticals.

between 0.3 and 3 Gyr going from the steepest CLOSE DD case, to the flattest WIDE DD case. The SD-Chandra model with $\gamma=0.005$ (green dashed line) has a typical timescale of $\simeq 1 \mathrm{Gyr}$, which is often taken as a reference value to infer the formation timescale of systems exhibiting an

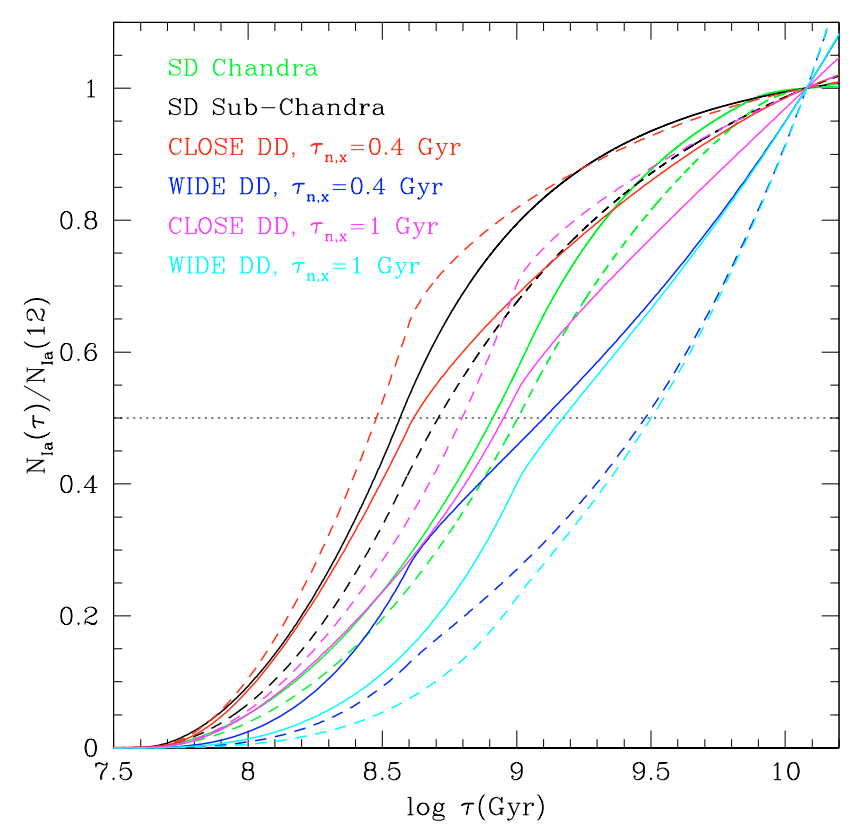

Fig. 13. Cumulative number of SNIa explosions following an instantaneous burst of SF for the same models shown in Fig. 11, with the same color and line coding. In particular, the dashed blue and cyan lines are especially flat WIDE $D D$ cases; the dashed red and magenta lines are especially steep CLOSE DD models.

$\alpha /$ Fe overabundance. Figure 13 emphasizes that such timescale does depends on the SNIa model, namely it is longer the flatter the distribution of the delay times of the SNIa progenitors is. The estimated formation timescales, then, remain uncertain by a factor of a few, modulo the actual SNIa channel that dominates in nature.

\subsection{SNla rate in different galaxy types}

As anticipated in the previous section, the large differences of the SNIa rate temporal behavior typical of the various models translate into a different trend of the SNIa rate as a function of the galaxy type, thereby offering a tool to discriminate among the potential progenitors. This has already been outlined in Sect. 2, where the Cappellaro et al. (1999) data have been shown to indicate that the ratio between the $f_{\text {Ia }}$ value at late delay times and its average value over the whole range of delay times (up to the Hubble time) should be $\simeq 0.15$. For the analytic functions, the quantity $\frac{\left\langle f_{\mathrm{la}}\right\rangle_{10,12}}{\left\langle f_{\mathrm{f}}\right\rangle_{0.12}}$ is equal to $0.02(0.03)$, 0.05 (0.08) respectively for the SD Chandra (Sub-Chandra) models with $\gamma=1$ and 0.005 ; equal to 0.07 and 0.15 for the CLOSE DDs with $\tau_{\mathrm{n}, \mathrm{x}}=0.4,1$ (and $\beta_{\mathrm{g}}=-0.75$ ); while it is $\simeq 0.23$ for the WIDE DDs with a flat distribution of the separations $\left(\beta_{\mathrm{a}}=0\right)$. Therefore, the ratio between the SNIa rate in Ellipticals and Spirals indicates that the Single Degenerate model underestimates the current rate in early type galaxies, with respect to the rate in late types, a result of its fast decline at late times.

This constraint has been derived considering a schematic description of the star formation history in early and late type galaxies, and by using a theoretical value for the 
$\mathcal{M} / L_{\mathrm{B}}$ ratio in the two galaxy types. A much better constraint on the SNIa model progenitors can be built upon the recent results by M2005, by considering the trend of the SNIa rate per unit galaxy mass with the parent galaxy type. In fact, Eq. (4) can be written as:

$$
\frac{\dot{n}_{\mathrm{Ia}}(t)}{\mathcal{M}}=k_{\alpha} \cdot A_{\mathrm{Ia}} \cdot\left\langle f_{\mathrm{Ia}}\right\rangle_{\psi(t)}
$$

where $\left\langle f_{\mathrm{Ia}}\right\rangle_{\psi(t)}$ is the average of the distribution function of the delay times weighted by the star formation rate over the galaxy lifetime, and where the integral of the SFR has been approximated with the galaxy (stellar mass) $\mathcal{M}^{9}$. Equation (40) clearly shows that the trend of the SNIa rate with galaxy type reflects the systematics of the their star formation histories. Given that $f_{\text {Ia }}$ is a decreasing function of the delay time, the younger systems will have a higher SNIa rate per unit mass, by an amount which depends on the shape of the $f_{\mathrm{Ia}}$ function. The correlation in M2005 can be interpreted as the result of Eq. (40), where the different SF histories imply both a different SNIa rate per unit mass, and a different $B-K$ color.

Figures 14 and 15 show the M2005 observed correlation, and the theoretical predictions for the various SNIa models, computed as follows. To describe the SF history in the various galaxy types I have considered four families of models:

(1) old burst models, characterized by a constant SFR within a starting epoch $t=0$ and ending epoch $t_{\mathrm{B}}=0.5,1,1.5$ and 2 Gyr;

(2) exponentially decreasing models, all starting at epoch $t=0$ and with different e-folding times $t_{\mathrm{SF}}: \psi(t)=\mathrm{e}^{-\frac{t}{t_{\mathrm{SF}}}}$ with $t_{\mathrm{SF}}=1,3,5,7,9,11 \mathrm{Gyr}$;

(3) exponentially increasing models: all starting at epoch $t=0$ and with a different characteristic time $\tau_{\star}: \psi(t)=\mathrm{e}^{-\frac{12-t}{\tau_{\star}}}$ with $\tau_{\star}=8,6,4,3,2,1$ Gyr. These models correspond to a sequence of age distributions all peaked at $12 \mathrm{Gyr}$, and decreasing e-folding age, so that the lower $\tau_{\star}$ the younger the average age of the stellar population;

(4) young burst models, characterized by a constant SFR within a starting epoch $t_{\star}=9.5,10,10.5,11 \mathrm{Gyr}$ and all ending at $t=12 \mathrm{Gyr}$.

In addition, a model with a constant SFR over the whole range of 12 Gyr has been computed. The considered range of SF histories appears to encompass the range of $B-K$ colors of the galaxies in M2005 sample. The composite colors have been computed using Girardi et al. (2000) simple stellar population models with solar metallicity. The bluest and reddest bin in M2005 are defined as lower and upper limits only (i.e. $B-K<2.6$ and $B-K>4.1$ ); here they have been specified so that they are close to the bluest and the reddest model stellar population. The point type in Figs. 14 and 15 encodes the SF history: burst models are plotted as circles; exponential models as crosses, each family ( 2 and 3 described above) connected by a line. The solitary dot shows the model with a constant SF rate over 12 Gyr.

\footnotetext{
9 As pointed out in Sect. 2, the actual stellar mass in a galaxy can be $\approx 30$ (45)\% lower than the integrated SFR for Salpeter (Kroupa) IMF, due to the mass return from dying stars.
}

The theoretical rates have been normalized so as to reproduce the observed values in the reddest galaxies with a SFH given by the old burst model with a duration of 2 Gyr; consequently, the normalization implies different realization probabilities of the SNIa event for the different models. The values for $A_{\text {Ia }}$ required by this normalization, having adopted a Kroupa IMF and Eq. (16) for the distribution function of the secondaries, are labeled in the figure. $A_{\text {Ia }}$ is the number fraction of SNIa events out of a stellar generation, and should be compared to the number fraction of stars in a mass range which can lead to the event, e.g. $3 \leq m / M_{\odot} \leq 8$. For Kroupa IMF, $20 \%$ of the stars fall in this mass range, and therefore the normalization requires that in the WIDE DD scenario, approximately $3 \%$ of the stars in the suitable mass range should end up as SNIa. For the CLOSE DD scenario the analogous fraction is around $\sim 10 \%$. The dependence of these figures on the parameters for the DD model can be appreciated from the values labeled in Fig. 14; for Salpeter IMF, the required fraction of SNIa events from the same mass range is smaller by a factor of $\sim 0.6$. It can be noticed that choosing any other old burst model, or the oldest exponentially decreasing model, would not change the normalization.

It appears that the both families of DD models do fit well the observational data when the choice of the parameters is such to provide an intermediate shape of the distribution function of the delay times. The CLOSE DDs yield a too steep evolution of the SNIa rate per unit mass with galaxy color, for a low $\beta_{\mathrm{g}}$ and a short $\tau_{\mathrm{n}, \mathrm{x}}$; similarly, the WIDE DDs give a too flat relation if $\beta_{\mathrm{a}}$ is large, as well as $\tau_{\mathrm{n}, \mathrm{x}}$. This comparison does not necessarily favor the WIDE or the CLOSE DDs; rather it points to a moderate solution: either relatively flat CLOSE DDs or relatively steep WIDE DDs.

Figure 15 shows the analogous results for the SD models. The left panel clearly shows that, with the standard choice of the parameters (i.e. $n\left(m_{2}\right)$ as in the population synthesis computations, $\alpha=2.35$ and $\gamma=1$ ) both Chandra and Sub-Chandra exploders imply a too large increase of the SNIa rate per unit mass going from early to late type galaxies. The right panel shows the results for a choice of the parameters aimed at improving the match between the SD model and the observations. When using Eq. (20) to describe $n\left(m_{2}\right)$ the agreement is better (green and magenta points), but is seems that only with a very low value of $\gamma$ (i.e. if all the mass ratios $q=m_{2} / m_{1}$ are equally probable) can the SD model be reconciled with the observations. In this case, the normalization to the rate in the reddest galaxies implies that $\sim 10 \%$ of all stars born with $2 \leq m / M_{\odot} \leq 8$ should end up as SNIa of the SD Chandra variety; alternatively $\sim 12 \%$ of all stars born with $3 \leq m / M_{\odot} \leq 8$ should end up as SD Sub-Chandra SNIa.

The comparison between the M2005 data and the theoretical models does not definitely rule out any of the alternative progenitors, but puts constraints on the key parameters within the various families. The precise values of these parameters are subject to some uncertainty. M2005 convert their observed SNIa rate per unit luminosity into a rate per unit mass by using the results from the galaxy models by Bell \& de Jong (2001), which are much more complicated than the SF histories considered here. This introduces a (small) inconsistency 


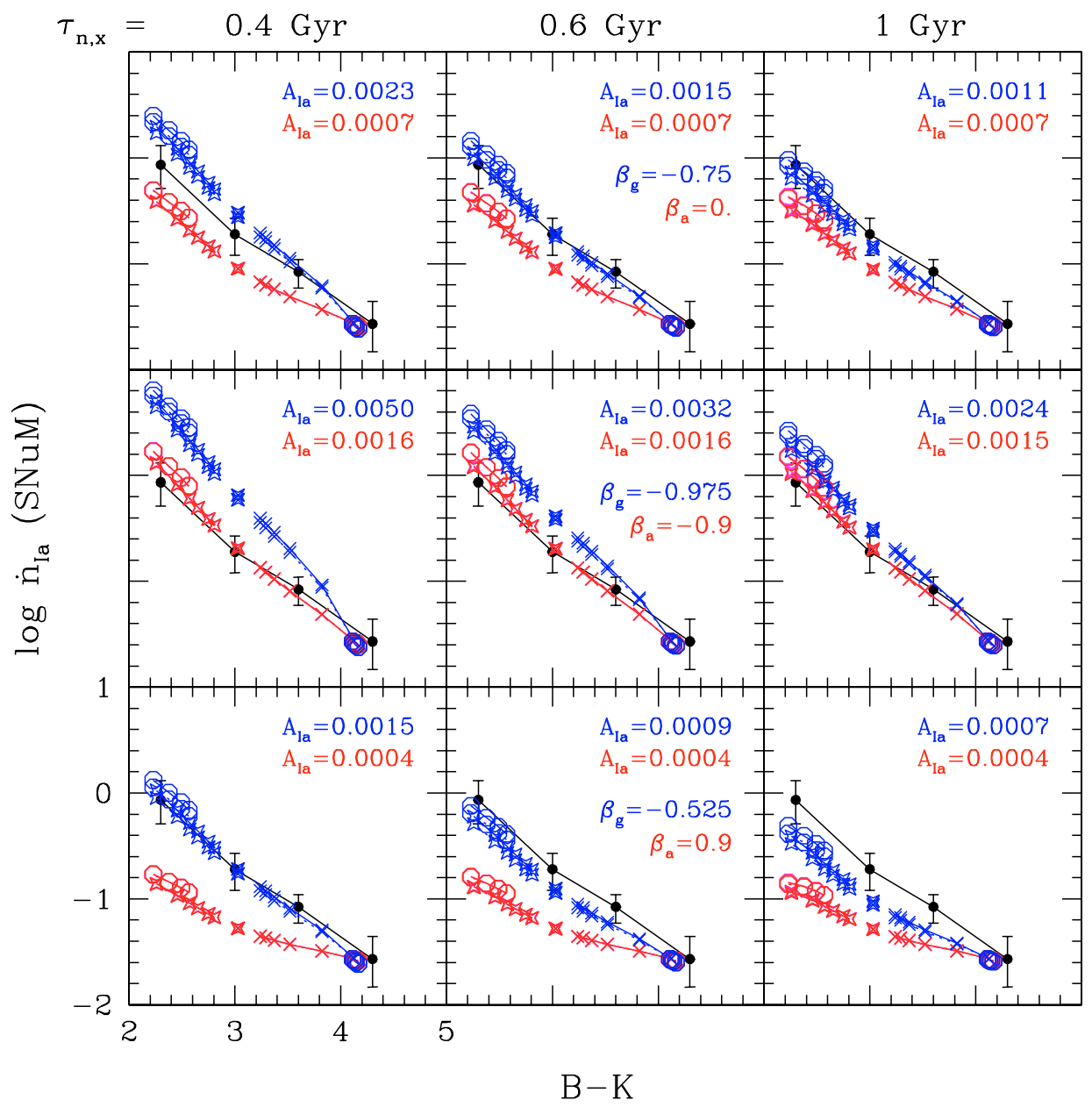

Fig. 14. Comparison between Double Degenerate model predictions and observations of the SNIa rate per unit mass as a function of the color of the parent galaxy. The data from M2005 paper are plotted as black dots, with their quoted error bars, and connected with a solid line. The 9 panels show the trend with $B-K$ as a tracer of SF history (see text) of CLOSE DD (blue) and WIDE DD (red) models: from left to right the minimum secondary mass in SNIa progenitor systems decreases, and its MS lifetime $\tau_{\mathrm{n}, \mathrm{x}}$ increases; from top to bottom the slope in the decline phase varies as indicated in the central panels, reflecting different distribution functions of the DD separations. Also labeled are the realization probabilities required by the different models in order to reproduce the current rate in the reddest galaxies. All models adopt $\alpha=2.35$ and $\gamma=1$, and both options for the distribution function of the secondaries, i.e. Eqs. (16) and (20) are displayed. The minimum gravitational delay is assumed to be negligible; the maximum gravitational delay for the CLOSE DDs follows Eq. (30). The two options for the WIDE DD case, i.e. $f_{1,2}^{\mathrm{W}}=f_{1}^{\mathrm{W}}$ and $f_{2}^{\mathrm{W}}$ in Eq. (36) give the same results, and indeed are both plotted in of the various panels.

between the models and the data. Another caveat concerns the approximation of the galaxy mass with the integrated SF rate, which affects the zero point (by not more than a factor of 2), and the slope of the theoretical trend. Both these approximations, however, hardly affect the major conclusion that indicates that

(i) the distribution function of the delay times should drop by a factor of $\approx 100$ from its early peak to its value at 10 Gyr;

(ii) in principle all candidates can reproduce the observed trend of the SNIa rate per unit mass with galaxy color, with an adequate combination of the key parameters. However, the SD channel seem to require quite some fine tuning; in particular the Chandra model is viable only if the accretion efficiency is very large, which appears unlikely.

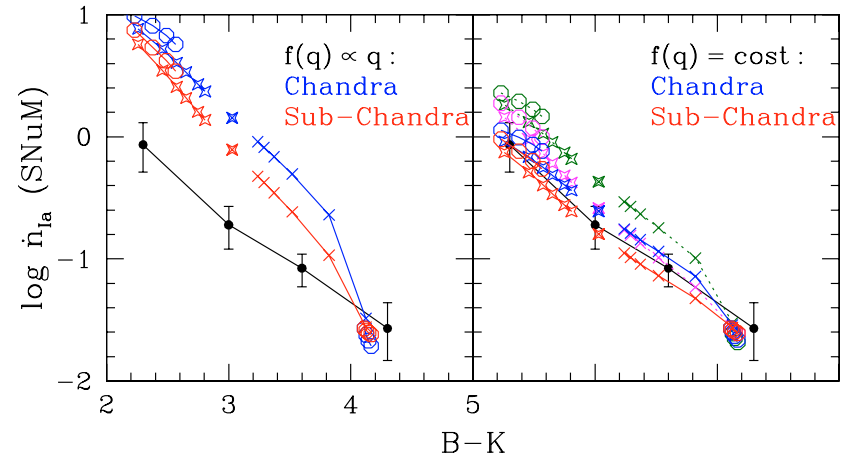

Fig. 15. Comparison between Single Degenerate model predictions and M2005. The point type encodes the SFH, as in Fig. 14. All models adopt $\alpha=2.35$; other parameters are labeled. The curves have been obtained adopting Eq. (16) (left) and Eq. (20) (right panel). The green and magenta loci show the results for $\gamma=1$. 


\section{Summary and concluding remarks}

In this paper I have presented a straightforward formalism to relate the rate of SNIa events in stellar systems to their star formation history through two fundamental characteristic of the SNIa progenitor model: the realization probability of the SNIa scenario from a single age stellar population $A_{\text {Ia }}$, and the distribution function of the delay times $f_{\mathrm{Ia}}(\tau)$, which is proportional to the SNIa rate past an instantaneous burst of star formation.

The various models for the SNIa progenitors correspond to different values for $A_{\text {Ia }}$, and to different shapes of the $f_{\mathrm{Ia}}$ function. No attempt is made here to give a theoretical value for $A_{\text {Ia }}$, which can be derived either from the numerical realizations in population synthesis models, or directly from the observations. The latter suggest $A_{\text {Ia }}$ on the order of $10^{-3}$ (see Sect. 2.1). A more precise estimate could be derived with a detailed modeling of the star formation in late type galaxies, and with a robust assessment of the IMF. For the $f_{\text {Ia }}$ function instead, the paper presents analytical formulations to describe the most popular SNIa progenitor models. It is shown that some parameters play a key role in shaping the distribution function of the delay times, most notably: the mass range of the secondaries in systems which provide SNIa events; the minimum mass of the primary which yields a massive enough CO WD to ensure the explosion, and the efficiency of accretion on top of it, for the SD model; the distribution of the separations of the DD systems at their birth. In addition, it is found that the algorithm adopted to describe the distribution of the stellar masses in binaries has an important impact on the slope of the $f_{\mathrm{Ia}}$ function for the SD model.

In all of the three scenarios (SD Chandra and Sub-Chandra, and DD Chandra) the distribution function of the delay times is characterized by a steep, early rise, so that the maximum rate is reached soon after the first SNIa explodes. For the SD model, this reflects the behavior of the clock $\left(\dot{m}_{2}\right)$ which describes how the rate of change of the evolutionary mass decreases as the delay time increases. The quantity $\left|\dot{m}_{2}\right|$ drops so fast to prevail over the increase of the number of SNIa precursors as the delay time increases. For the DD model, the clock has an additional contribution from the gravitational delay; still the early steep rise is present because most DD systems have a short $\tau_{\mathrm{gw}}$, given that a wide range of masses and separations correspond to a short gravitational delay. Therefore, the property of the distribution function of the delay times to reach its maximum shortly after the first event occurs has a very robust justification. As repeatedly said, following an instantaneous burst of SF, the epoch of the first event is equal to the MS lifetime of the most massive secondary $\left(\tau_{\mathrm{n}, \mathrm{i}}\right)$, for the SD model, plus the minimum gravitational delay $\left(\tau_{\mathrm{gw}, \mathrm{i}}\right)$, for the DD model. There's no apparent reason to consider an upper limit for $m_{2}$ in SNIa progenitor systems smaller than the most massive primary which provides a CO WD (i.e. an $\sim 8 M_{\odot}$ star). This implies $\tau_{\mathrm{n}, \mathrm{i}} \simeq 0.04 \mathrm{Gyr}$. As shown in Fig. 4, even in the WIDE DD scheme the binary WDs can emerge from the CE with a very small separation, so that also $\tau_{\mathrm{gw}, \mathrm{i}}$ is likely to be very short. Therefore, in the context of the binary evolution, it seems very hard to accommodate a shape for the distribution of the delay times similar to the one that according to Strolger et al. (2004) best explains the redshift dependence of the SNIa rates measured in GOODs, i.e. a Gaussian distribution centered on $\tau \sim 3-4$ Gyr. Such a distribution could be obtained if SNIa events were produced only by systems with $m_{2}$ in the vicinity of $1.3 M_{\odot}$, for the SD model, or by DD systems born with a separation of about $2.5 R_{\odot}$ (but notice that a little spread of the binary mass implies anyway a large spread of delays). Both hypothesis are very contrived. Furthermore, a Gaussian distribution would not meet the constraint on the $f_{\text {Ia }}$ function derived from the trend of the SNIa rate with parent galaxy type, like in M2005.

The analytical functions derived in this paper compare very well to the results of the population synthesis codes in the literature, and especially to those in Yungelson \& Livio (2000), when taking into account the appropriate mass ranges and kind of progenitor. The Monte Carlo simulations follow the evolution of a population of binaries, which evolve through mutually exclusive channels, according to the response of the system to the mass exchange phases. The remarkable similarity between the analytic functions derived here and the numerical results suggests that the shape of $f_{\text {Ia }}$ is mainly determined by the mass ranges and general characteristics of the clock of the explosion, while the details of the response of the individual systems to the RLO events are of lesser importance.

Once put on the same formalism, the various models for the SNIa progenitors can be compared to the relevant observations in order to judge which one best accounts for the data. The most direct observational counterpart of the $f_{\mathrm{Ia}}$ functions is the redshift dependence of the SNIa rate in elliptical galaxies (per unit galaxy mass), due to the fact that the bulk of their stars formed at very early epoch, and the single burst is a reasonable approximation. Along this line, Maoz \& Gal-Yam (2004) have compared the SNIa rate in galaxy clusters at redshift between 0 and 1 to the family of $f_{\text {Ia }}$ functions proposed by Madau et al. (1998), reaching the conclusion that the average delay time of SNIa precursors ought to be $\leq 2$ Gyr. The notion of a typical delay time for the SNIa precursors has very little justification in the context of stellar evolution in binaries, since a wide range of delay times is produced by any kind of progenitor. Rather, the slow increase of the SNIa rate with redshift reported in Maoz \& Gal-Yam (2004) would favor the DD model, perhaps better of the WIDE DD variety. With the progress of the Cluster SN surveys (see e.g. Maoz 2005) it will be possible to further investigate the redshift dependence of the rate of SNIa in Elliptical galaxies, and come to stronger conclusions. A less direct, but definitely complementary and effective approach to constrain the SNIa progenitor model is attempted here, by considering the predicted trend of the SNIa rate (per unit mass) with the parent galaxy type (see also Della Valle \& Livio 1994; Ruiz-Lapuente et al. 1995). The M2005 data suggest that the DD channel is favored with respect to the SD channel, and that the distribution of the separations of the DD systems should be such to produce a moderate decline of the $f_{\text {Ia }}$ function at late times. The SD model is not completely ruled out by this comparison, but it requires (i) a flat distribution of the mass ratios and (ii) accretion efficiencies close to $100 \%$. This can be accomplished only if the matter is accreted and burned on top of the WD at the same pace, so as to avoid either 
expansion beyond the Roche Lobe (and the formation of a Common Envelope), or the accumulation of a Hydrogen layer which is eventually ignited under degenerate conditions (so that a Nova explosion occurs). Even in the Hachisu et al. (1996) scenario part of the accreted matter is lost by the system in a stellar wind.

The various models for the SNIa progenitors have different impact on the large scales; some preliminary considerations are in Greggio (2005), while more detailed investigations will be presented elsewhere. Here I just remark a few points.

Once normalized to reproduce the current SNIa rate in Ellipticals, the SD model corresponds to a large Fe mass to light ratio in Cluster of Galaxies (see Fig. 13; and Greggio 2005). A detailed study of the expected Fe mass to light ratio in galaxy clusters as a function of the various possible progenitors, and its evolution with redshift will allow us to better constrain the SNIa model and the contribution of SNIa to the Fe in the intracluster medium.

The timescale over which, following an instantaneous burst of SF, half of the $\mathrm{Fe}$ is released to the interstellar medium varies between $\sim 0.3$ to $3 \mathrm{Gyr}$ for the wide variety of SNIa progenitor models considered here. Accordingly, the formation timescale of systems which exhibit an enhancement of $\alpha$ elements with respect to $\mathrm{Fe}$ is rather uncertain, and depends on the SNIa model. Quantitatively, the actual constraint on the formation timescales also varies with the duration of the star formation episode in the system (Matteucci \& Recchi 2001). Preliminary computations show that, in a star forming system, such timescale may range between 1 and several Gyr. This problem will be discussed in a forthcoming paper.

The evolution of the gas flows in Ellipticals depends on the balance between the rate of mass return and the SNIa rate, past a burst of SF. The former scales with time as $\propto t^{-1.3}$, while, at delay times greater than $\sim 1 \mathrm{Gyr}$, the $f_{\text {Ia }}$ functions scale as $\propto t^{-s}$ with $s \sim-1$ for the DDWIDE, - 1.2 for the DD CLOSE, $\sim-1.6$ for the SD Sub-Chandra. Therefore, it appears that the secular evolution of the SNIa rate past a burst of SF is critically close to the evolution of the rate of mass return, and that the fate of the gas in Ellipticals is very sensitive to the SNIa progenitor's model. It is also worth noticing that the shape of the $f_{\text {Ia }}$ function is different from a simple power law, as is adopted in Ciotti et al. (1991) to model the gas flows in ellipticals. In particular, the presence of the wide maximum phase at intermediate epochs will impact on the dynamical evolution of the gas.

In this paper, the emphasis has been put on the intercomparison of the various models for the SNIa progenitor. Actually, all different channels could contribute to the SNIa events, each with its own probability, as in the realizations of the population synthesis models. Some diversities of the observational properties of SNIa have been found in the literature, which support this notion (e.g. Branch 2004; Benetti et al. 2005). In particular the different luminosity at maximum, and the different decline rate of the light curve, as measured by the the $\Delta m_{15}$ parameter of Phillips (1993), of the events in early and late type galaxies (Della Valle \& Panagia 1992; van den Bergh \& Pazder 1992; Hamuy et al. 1996; Garnavich \& Gallagher 2005) could be related to different typical progenitors. If both the Single and Double Degenerate channels are at work with similar total realization probabilities, in early type galaxies the DD explosions should prevail over SD events, since the distribution function of the delay times of the latter declines fast. In late type galaxies, instead, all channels should contribute to the current rate, with a larger proportion of SNIa from the SD channel, due to their high rate at early epochs. The formalism presented in this paper allows a straightforward exploration of the effect of a mixture of progenitors, e.g. by varying the relative $A_{\text {Ia }}$ realization probabilities. Eventually, it will be possible to constrain the mixture of progenitors by modeling the evolution of the SNIa rate in galaxies of different types, and considering as well all the other consequences on the large scales.

Acknowledgements. I am indebted to Alvio Renzini for a critical reading of the manuscript and many useful suggestions. I also thank Luca Ciotti for discussions on the mathematical formalism, and Francesca Matteucci and Simone Recchi for having renewed my interest in the problem of SNIa theoretical rates. This work was partly supported by the Italian Ministery of University and Research (MURST) under the grant COFIN 2003.

\section{References}

Bell, E. F., \& De Jong, R. S. 2001, ApJ, 550, 212

Benetti, S., Cappellaro, E., Mazzali, P. A., et al. 2005, ApJ, 623, 1011

Bragaglia, A., Greggio, L, Renzini, A., \& D’Odorico, S. 1990, ApJ, 365, L13

Branch, D. 2004, in Cosmic explosions in 3 dimensions: asymmetries in Supernovae and gamma-ray bursts, ed. P. Hoflich, P. Kumar, \& J. C. Wheeler (Cambridge: CUP), 132

Cappellaro, E., Evans, R., \& Turatto, M. 1999, A\&A, 351, 459

Ciotti, L., D'Ercole, A., Pellegrini, S., \& Renzini, A. 1991, ApJ, 376, 380

De Donder, E., \& Vanbeveren, D. 2003, New Astron., 8, 817

Della Valle, M., \& Panagia, N. 1992, AJ, 104, 696

Della Valle, M., \& Livio, M. 1994, ApJ, 423, L31

Eggleton, P. P. 1983, ApJ, 268, 368

Fedorova, A. V., Tutukov, A. V., \& Yungelson, L. R. 2004, Astron. Lett., 30, 73

Foss, D., Wade, R. A., \& Green, R. F. 1991, ApJ, 374, 281

Garnavich, P. M., \& Gallagher, J. 2005, in 1604-2004: Supernovae as Cosmological Lighthouses, ASP Conf. Ser., in press [arXiv: astro-ph/0501065]

Girardi, L., Bressan, A., Bertelli, G., \& Chiosi, C. 2000, A\&AS, 141, 371

Greggio, L. 1996, in The Interplay between Massive Star Formation, the ISM, and Galaxy Evolution, ed. D. Kunth et al. (Gif-surYvette: Éditions Frontières), 98

Greggio, L. 1997, MNRAS, 285, 151

Greggio, L. 2005, in 1604-2004: Supernovae as Cosmological Lighthouses, ASP Conf. Ser., in press [arXiv: astro-ph/0410187]

Greggio, L., \& Renzini, A. 1983, A\&A, 118, 217

Greggio, L., \& Renzini, A. 1990, ApJ, 364, 35

Hachisu, I., \& Kato, M. 2001, ApJ, 558, 323

Hachisu, I., Kato, M., \& Nomoto, K. 1996, ApJ, 470, L97

Hachisu, I., Kato, M., \& Nomoto, K. 1999, ApJ, 522, 487

Hamuy, M., Phillips, M. M., Suntzeff, N. B., et al. 1996, AJ, 112, 2391

Han, Z., Podsiadlowski, Ph., \& Eggleton, P. P. 1995, MNRAS, 272, 800

Han, Z. 1998, MNRAS, 296, 1019

Han, Z., \& Podsiadlowski, Ph. 2004, MNRAS, 350, 130 
Iben, I., Jr., \& Tutukov, A. V. 1984, ApJS, 54, 335

Iben, I., Jr., \& Tutukov, A. V. 1985, ApJS, 58, 661

Landau, L. D., \& Lifshitz, E. M. 1962, in The Classical Theory of Fields (Oxford: Pergamon)

Kenyon, S. J., Livio, M., Mikolajewska, J., \& Tout, C. A. 1993, ApJ, 407, L81

Kroupa, P. 2001, MNRAS, 322, 231

Livio, M. 2001, in Supernovae and gamma-ray bursts: the greatest explosions since the Big Bang, ed. M. Livio, N. Panagia, \& K. Sahu (Cambridge: CUP), 334

Loewenstein, M., \& Mathews, W. G. 1987, ApJ, 319, 614

Madau, P., Della Valle, M., \& Panagia, N. 1998, MNRAS, 297, L17

Mannucci, F., Della Valle, M., Panagia, N., et al. 2005, A\&A, 433, 807 (M2005)

Maoz, D., \& Gal-Yam, A. 2004, MNRAS, 347, 951

Maoz, D. 2005, in 1604-2004: Supernovae as Cosmological Lighthouses, ASP Conf. Ser., in press [arXiv: astro-ph/0501492]

Maraston, C. 1998, MNRAS, 300, 872

Maraston, C. 2005, MNRAS, in press [arXiv: astro-ph/0410207]

Matteucci, M. F. 1994, A\&A, 288, 57

Matteucci, M. F., \& Greggio, L. 1986, A\&A, 154, 279

Matteucci, M. F., \& Vettolani, G. 1988, A\&A, 202, 21

Matteucci, M. F., \& Recchi, S. 2001, ApJ, 558, 351

Maxted, P. F. L., \& Marsh, T. R. 1999, MNRAS, 307, 122

Munari, U., \& Renzini, A. 1992, ApJ, 397, L87

Napiwotzki, R., Christlieb, N., Drechsel, H., et al. 2001, AN, 322, 411

Napiwotzki, R., Yungelson, L. R., Nelemans, G., et al. 2004, in Spectroscopically and spatially resolving the components of close binary stars, ed. R. W. Hilditch, H. Hensberge, \& K. Pavlovski, ASP Conf. Ser., 402

Nelemans, G., Verbunt, F., Yungelson, L. R., \& Portegies-Zwart, S. F. 2000, A\&A, 360, 1011

Nelemans, G., Yungelson, L. R., Portegies-Zwart, S. F., \& Verbunt, F. 2001, A\&A, 365, 491

Nelemans, G., \& Tout, C. A. 2005, MNRAS, 356, 753

Nomoto, K. 1982, ApJ, 253, 798

Peebles, P. J. E. 2002, in A New Era in Cosmology, ed. N. Metcalfe, \& T. Shanks, ASP Conf. Ser., 283, 351
Perlmutter, S., Aldering, G., Goldhaber, G., et al. 1999, ApJ, 517, 565

Phillips, M. M. 1993, ApJ, 413, L105

Piersanti, L., Gagliardi, S., Iben, I., Jr., \& Tornambé, A. 2003, ApJ, 598, 1229

Rappaport, S., Di Stefano, R., \& Smith, J. D. 1994, ApJ, 426, 692

Rasio, F. A., \& Livio, M. 1996, ApJ, 471, 366

Renzini, A. 1996, in New Light on Galaxy Evolution, ed. R. Bender, \& R. L. Davies (Dordrecht: Kluwer), 131

Renzini, A. 1997, ApJ, 488, 35

Renzini, A. 1999, in The formation of galactic Bulges, ed. C. M. Carollo, H. C. Ferguson, \& R. F. G. Wise (Cambridge: CUP), 9

Robinson, E. L., \& Shafter, A. W. 1987, ApJ, 322, 296

Riess, A. G., Filippenko, A. V., Challis, P., et al. 1998, AJ, 116, 1009

Ruiz-Lapuente, P., Burkert, A., \& Canal, R. 1995, ApJ, 447, L69

Ruiz-Lapuente, P., \& Canal, R. 1998, ApJ, 497, L57

Ruiz-Lapuente, P., Comeron, F., Mendéz, J., et al. 2004, Nature, 431, 1069

Saffer, R. A., Livio, M., \& Yungelson, L. R. 1998, ApJ, 502, 394

Saio, H., \& Nomoto, K. 1998, ApJ, 500, 388

Saio, H., \& Nomoto, K. 2004, ApJ, 615, 444

Strolger, L. G., Riess, A. G., Dahlen, T., et al. 2004, ApJ, 613, 200

Thomas, D., Maraston, C., Bender, R., \& Mendes de Oliveira, C. 2005, ApJ, 621, 673

Tinsley, B. M. 1980, Fund. Cosmic Phys., 5, 287

Tutukov, A. V., \& Yungelson, L. R. 1994, MNRAS, 268, 871

van den Bergh, S., \& Pazder, J. 1992, ApJ, 390, 34

Van den Heuvel, E. P. J., Bhattacharya, D., Nomoto, K., \& Rappaport, S. A. 1992, A\&A, 262, 97

Webbink, R. F. 1984, ApJ, 277, 355

Whelan, J., \& Iben, I., Jr. 1973, ApJ, 186, 1007

Williams, K. A., Bolte, M., \& Koester, D. 2004, ApJ, 615, L49

Woosley, S. E., \& Weaver, T. A. 1994, ApJ, 423, 371

Yungelson, L. R. 2004, in Intergalactic Binaries: Accretion, Evolution and Outcomes, AIP, ed. L. A. Antonelli, L. Burderi, \& F. D'Antona et al., in press [arXiv: astro-ph/0412635]

Yungelson, L. R., Livio, M., Tutukov, A. V., \& Saffer, R. A. 1994, ApJ, 420, 336

Yungelson, L. R., \& Livio, M. 2000, ApJ, 528, 108 (YL) 


\section{Online Material}




\section{Appendix A: Equations used to derive the $f_{\mathrm{la}}^{\mathrm{DD}}(\tau)$ function}

\section{A.1. An approximate relation for the gravitational delay}

The gravitational delay time is given by:

$$
\tau_{\mathrm{gw}}=\frac{0.15 A^{4}}{\left(m_{1 \mathrm{R}}+m_{2 \mathrm{R}}\right) m_{1 \mathrm{R}} m_{2 \mathrm{R}}} \mathrm{Gyr} .
$$

The mass dependent term can be written as

$$
f=M_{\mathrm{DD}}^{2} \cdot m_{2 \mathrm{R}}-m_{2 \mathrm{R}}^{2} \cdot M_{\mathrm{DD}}
$$

with $M_{\mathrm{DD}}=m_{1 \mathrm{R}}+m_{2 \mathrm{R}}$, i.e. the total mass of the DD system. Given that WD masses range between 0.6 and $1.2 M_{\odot}$, $M_{\mathrm{DD}}$ goes from 1.2 to $2.4 M_{\odot} . f$ is a family of parabolas, both viewed as a function of $m_{2 \mathrm{R}}$, and of $M_{\mathrm{DD}}$. These families are shown in Fig. (A.1). Each parabola in panel (a) has an absolute maximum in $m_{2 \mathrm{R}}=0.5 M_{\mathrm{DD}}$, of $f_{\max }=0.25 \cdot M_{\mathrm{DD}}^{3}$. This locus is drawn in panel (a) as the dashed line, and in panel (b) as the uppermost thick curve. Not all combinations of $\left(M_{\mathrm{DD}}\right.$, $m_{2 \mathrm{R}}$ ) are acceptable, and the shaded area in the two panels show the allowed parameter space. In addition to the vertical limits $1.4 \leq M_{\mathrm{DD}} / M_{\odot} \leq 2.4$, and $0.6 \leq m_{2 \mathrm{R}} / M_{\odot} \leq 1.2$, it is further required that $m_{1 \mathrm{R}}\left(=M_{\mathrm{DD}}-m_{2 \mathrm{R}}\right)$ ranges between $0.6 M_{\odot}$ and $1.2 M_{\odot}$. The more restrictive criterion $m_{2 \mathrm{R}} \leq m_{1 \mathrm{R}} \leq 1.2 M_{\odot}$ is unnecessary. Notice that, occasionally, the evolution in Close Binaries produces $m_{1 \mathrm{R}}<m_{2 \mathrm{R}}$ (even though, by definition, $m_{1} \geq m_{2}$ ), e.g. in some cases of conservative RLO when the secondary may become more massive than the original primary, thereafter leaving a more massive WD remnant.

The loci $m_{1 \mathrm{R}}=0.6 M_{\odot}$ and $m_{1 \mathrm{R}}=1.2 M_{\odot}$ are shown in Fig. A.1: along each parabola, only the portion included between the two loci is acceptable. Basically, at given $M_{\mathrm{DD}}$ we exclude those values of $m_{2 \mathrm{R}}$ which imply $m_{1 \mathrm{R}}<0.6 M_{\odot}$, which is a He WD; similarly, we exclude those values of $m_{2 \mathrm{R}}$ which imply $m_{1 \mathrm{R}}>1.2 M_{\odot}$, taken here as the upper limit to the mass of a WD.

As clearly visible in panel b) of Fig. A.1, the term $f$ is much more sensitive to $M_{\mathrm{DD}}$ rather than to $m_{2 \mathrm{R}}$, and there is an almost one to one correspondence between $M_{\mathrm{DD}}$ and $f$ over the whole parameter space of double CO WDs. In the computations presented here I approximate the function $f$ with its maximum value of $0.25 \cdot M_{\mathrm{DD}}^{3}$, which is the upper envelope of the family of parabolas in panel (b). This approximation leads to Eq. (22).

\section{A.2. Shrinkage following the RLO}

Given the uncertainty of the results of the Common Envelope evolution I have considered two alternatives. In one case, both mass transfer phases are regulated through the standard $\mathrm{CE}$ Eq. (26), which results into:

$$
\frac{A_{\mathrm{f}}}{A_{\mathrm{i}}}=0.5 \alpha_{\mathrm{ce}} \frac{m_{\mathrm{f}}^{\mathrm{d}} m}{m_{\mathrm{i}}^{\mathrm{d}}}\left(\frac{m_{\mathrm{i}}^{\mathrm{d}}-m_{\mathrm{f}}^{\mathrm{d}}}{r_{\mathrm{L}}}+0.5 \alpha_{\mathrm{ce}} m\right)^{-1}
$$

where $m^{\mathrm{d}}$ indicates the mass of the donor, $A$ indicates the separation of the system, and the subscripts $i$ and $f$ refer to before

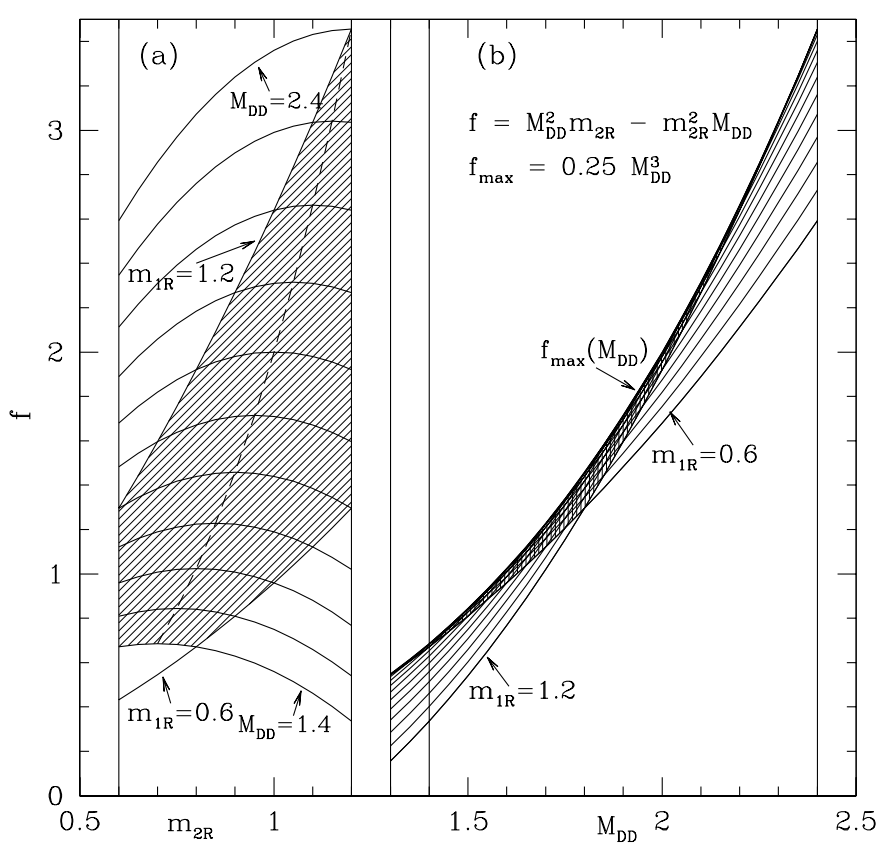

Fig. A.1. Mass dependent term of $\tau_{\mathrm{gw}}$ plotted versus $m_{2 \mathrm{R}}$ for selected values of $M_{\mathrm{DD}}$ (panel a)), and plotted versus $M_{\mathrm{DD}}$ for selected values of $m_{2 \mathrm{R}}$ (panel b)). The dashed curve in panel a) connects the maxima of the parabolas. Along this line $m_{2 \mathrm{R}}=m_{1 \mathrm{R}}$. The shaded area delimits the parameter space of SNIa producers.

and after the RLO, respectively. Further, $m$ is the mass of the companion, and $r_{\mathrm{L}}$ is the Roche Lobe radius in units of the initial separation, which is adopted from Eggleton (1998), and is generally $\sim 0.4-0.5$. The blue and cyan dots in Fig. 4 result from the twofold application of this equation, first with

$m_{\mathrm{i}}^{\mathrm{d}}=f_{\mathrm{en}} \cdot m_{1}, m=m_{2}$, and $m_{\mathrm{f}}^{\mathrm{d}}=m_{1 \mathrm{R}}$ as given by Eq. (19); second with

$m_{\mathrm{i}}^{\mathrm{d}}=f_{\mathrm{en}} \cdot m_{2}, m=m_{1 \mathrm{R}}$, and $m_{\mathrm{f}}^{\mathrm{d}}=m_{2 \mathrm{R}}$ as given by Eq. (19). In the alternative evolutionary scheme, the first mass transfer is considered parametrized by the Envelope Ejection relation in Nelemans et al. (2001), reported here as Eq. (27). Since, in the same notation adopted above:

$J_{\mathrm{i}}=m_{\mathrm{i}}^{\mathrm{d}} m \sqrt{\frac{G A_{\mathrm{i}}}{m_{\mathrm{i}}^{\mathrm{d}}+m}}, \quad J_{\mathrm{f}}=m_{\mathrm{f}}^{\mathrm{d}} m \sqrt{\frac{G A_{\mathrm{f}}}{m_{\mathrm{f}}^{\mathrm{d}}+m}}$

$\Delta M=m_{\mathrm{i}}^{\mathrm{d}}-m_{\mathrm{f}}^{\mathrm{d}}, \quad M_{\mathrm{B}}=m_{\mathrm{i}}^{\mathrm{d}}+m$.

it follows

$\frac{A_{\mathrm{f}}}{A_{\mathrm{i}}}=\left(\frac{m_{\mathrm{i}}^{\mathrm{d}}}{m_{\mathrm{f}}^{\mathrm{d}}}\right)^{2} \frac{m_{\mathrm{f}}^{\mathrm{d}}+m}{m_{\mathrm{i}}^{\mathrm{d}}+m}\left(1-\gamma \frac{m_{\mathrm{i}}^{\mathrm{d}}-m_{\mathrm{f}}^{\mathrm{d}}}{m_{\mathrm{i}}^{\mathrm{d}}+m}\right)^{2}$.

Equation (A.3) is applicable if

$\gamma \frac{m_{\mathrm{i}}^{\mathrm{d}}-m_{\mathrm{f}}^{\mathrm{d}}}{m_{\mathrm{i}}^{\mathrm{d}}+m} \leq 1$

otherwise the system would loose more than $100 \%$ of its original angular momentum. With a little algebra, the 
last relation becomes:

$\frac{m}{m_{\mathrm{i}}^{\mathrm{d}}} \geq \tilde{q}=\gamma\left(1-\frac{m_{\mathrm{f}}^{\mathrm{d}}}{m_{\mathrm{i}}^{\mathrm{d}}}\right)-1$

that is a lower limit to the mass ratio of the system at RLO. Typically, the quantity $\left(m_{\mathrm{f}}^{\mathrm{d}} / m_{\mathrm{i}}^{\mathrm{d}}\right.$ ) (which is the core mass fraction of the donor at RLO) ranges between 0.15 to 0.25 for $m_{\mathrm{i}}^{\mathrm{d}}$ between 8 and $2 M_{\odot}$. Then, $\tilde{q} \simeq 0.2,0.4,0.6$ for $\gamma=$ $1.5,1.75,2$. Close to this lower limit, the $A_{\mathrm{f}} / A_{\mathrm{i}}$ ratio from Eq. (A.3) becomes very small, and indeed smaller than that given by Eq. (A.2).

The red and magenta points in Fig. 4 are generated with the following prescriptions: similar to Nelemans et al. (2001), the first mass transfer results into a shrinkage given by Eq. (A.2) if the mass ratio is smaller than $\tilde{q}$, or the maximum $A_{\mathrm{f}} / A_{\mathrm{i}}$ from Eqs. (A.2) and (A.3) if the mass ratio is larger than $\tilde{q}$. At the second mass transfer, Eq. (A.2) is applied.

The product of the two $A_{\mathrm{f}} / A_{\mathrm{i}}$ ratios resulting from the first and the second mass transfer phases naturally equals the ratio between the final and the original separation of the close binary $\left(A / A_{0}\right)$ plotted in Fig. 4.

\section{A.3. Function $g\left(\tau, \tau_{n}\right)$ for WIDE DDs}

In this section I derive an expression for the fraction of systems which, having a nuclear delay $\tau_{\mathrm{n}}$, have a total delay smaller than $\tau$ for the WIDE DD evolutionary scenario. Under the assumptions that the total binary mass $\left(M_{\mathrm{DD}}\right)$ and the separation of the DD system $(A)$ are independent variables, the contribution to gravitational delay $\tau_{\mathrm{gw}}$ from systems with separations in the range $(A, A+\mathrm{d} A)$ is:

$\mathrm{d} n\left(\tau_{\mathrm{gw}}, A\right)=n(A) n\left(M_{\mathrm{DD}}\right) \mathrm{d} A \mathrm{~d} M_{\mathrm{DD}}$

where $n(A)$ and $n\left(M_{\mathrm{DD}}\right)$ are the distribution functions of the separations and total binary mass of the progeny of systems born with a secondary mass whose nuclear timescale is equal to $\tau_{\mathrm{n}}$, and the variables combine so that $\tau_{\mathrm{gw}}=0.6 \frac{A^{4}}{M_{\mathrm{DD}}^{3}}$. It follows:

$n\left(\tau_{\mathrm{gw}}\right) \mathrm{d} \tau_{\mathrm{gw}}=\mathrm{d} \tau_{\mathrm{gw}} \int_{A_{\mathrm{n}}}^{A_{\mathrm{x}}} n(A) n\left(M_{\mathrm{DD}}\right)\left|\frac{\partial M_{\mathrm{DD}}}{\partial \tau_{\mathrm{gw}}}\right| \mathrm{d} A$

where $A_{\mathrm{n}}$ and $A_{\mathrm{x}}$ define the range of separations which lead to the same gravitational delay $\tau_{\mathrm{gw}}$. Given the relation between gravitational delay, DD mass and separation, $A_{\mathrm{n}}$ and $A_{\mathrm{x}}$ are directly related to the minimum and maximum $M_{\mathrm{DD}}$ :

$A_{\mathrm{n}}=\frac{M_{\mathrm{DD}, \mathrm{n}}^{0.75} \tau_{\mathrm{gw}}^{0.25}}{0.6^{0.25}}, A_{\mathrm{x}}=\frac{M_{\mathrm{DD}, \mathrm{x}}^{0.75} \tau_{\mathrm{gw}}^{0.25}}{0.6^{0.25}}$.

Figure A. 2 shows plausible limits for $M_{\mathrm{DD}}$, illustrating that the heavier $m_{2}$ is, the heavier its $M_{\mathrm{DD}}$ progeny. To proceed, formulations for $n(A)$ and $n\left(M_{\mathrm{DD}}\right)$ need to be specified: since the WIDE $D D$ case is meant to describe a situation in which the evolution produces DDs in a wide range of separations, and since the relevant range of final separations is rather narrow (from 0.5 to $4.5 R_{\odot}$ ), as a convenient parametrization I adopt:

$n(A) \propto A^{\beta_{\mathrm{a}}}$.

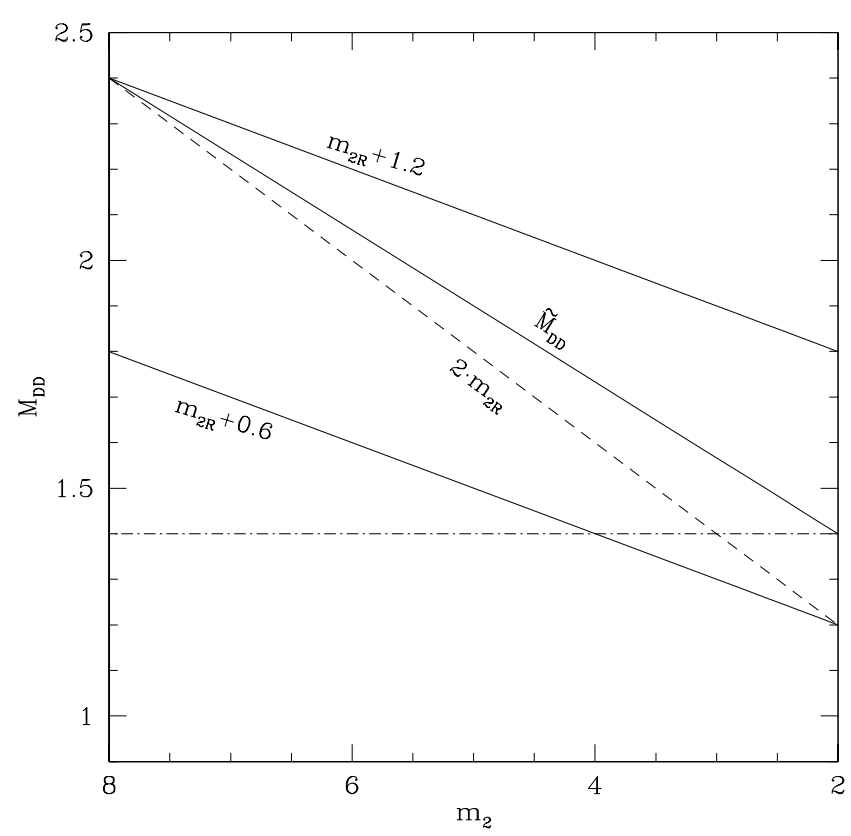

Fig. A.2. Limits for the total mass of the DD systems produced by primordial binaries with secondary mass $m_{2}$, leaving a remnant $m_{2 \mathrm{R}}$. The line labeled $\tilde{M}_{\mathrm{DD}}$ is used in the computations to explore the effect of a tight correlation between $m_{2}$ and its progeny $M_{\mathrm{DD}}$.

As for the distribution of the DD masses, I explore the consequences of two extreme assumptions:

(1) all values are equally probable: $n\left(M_{\mathrm{DD}}\right)=$ const. within

$M_{\mathrm{DD}, \mathrm{n}}=\max \left(1.4, m_{2 \mathrm{R}}+0.6\right) ; \quad M_{\mathrm{DD}, \mathrm{x}}=m_{2 \mathrm{R}}+1.2$

with $m_{2 \mathrm{R}}$ given by Eq. (19);

(2) the distribution is peaked around the value ${ }^{10}$ (see Fig. A.2):

$\tilde{M}_{\mathrm{DD}}=1.4+\left(m_{2}-2\right) / 6$.

With respect to case (1), notice that, although one could enforce $M_{\mathrm{DD}, \mathrm{n}}=2 m_{2 \mathrm{R}}$, there are evolutionary paths which could lead to $m_{2 \mathrm{R}}>m_{1 \mathrm{R}}$, e.g. when after the Ist RLO the separation is larger than the primordial one.

Case (2) corresponds to assuming that there is a tight correspondence between $m_{2}$ and the mass of the DD system, so that, at any $m_{2}$ (i.e. $\left.\tau_{\mathrm{n}}\right), M_{\mathrm{DD}}$ is specified, and the distribution of the gravitational delays of systems with given $\tau_{\mathrm{n}}$ is:

$n\left(\tau_{\mathrm{gw}}\right) \mathrm{d} \tau_{\mathrm{gw}} \propto n(A) \mathrm{d} A \quad$ with $A=\left(\tilde{M}_{\mathrm{DD}}\right)^{0.75} \tau_{\mathrm{gw}}^{0.25} / 0.6^{0.25}$.

With some algebra, Eq. (A.5) yields (for $\beta_{\mathrm{a}} \neq-1$ ):

$n\left(\tau_{\mathrm{gw}}\right) \propto f_{1,2}^{\mathrm{W}} \cdot \tau_{\mathrm{gw}}^{0.25 \beta_{\mathrm{a}}-0.75}$

where

$f_{1}^{\mathrm{W}} \propto \tilde{M}_{\mathrm{DD}}^{0.75+0.75 \beta_{\mathrm{a}}}$

for a narrow distribution of $M_{\mathrm{DD}}$ around $\tilde{M}_{\mathrm{DD}}$, while

$f_{2}^{\mathrm{W}} \propto M_{\mathrm{DD}, \mathrm{x}}^{1.75+0.75 \beta_{\mathrm{a}}}-M_{\mathrm{DD}, \mathrm{n}}^{1.75+0.75 \beta_{\mathrm{a}}}$

for a wide distribution of $M_{\mathrm{DD}}$.

\footnotetext{
${ }^{10}$ This arbitrary relation is used just to explore the effect of a systematic decrease of $M_{\mathrm{DD}}$ as $m_{2}$ decreases.
} 


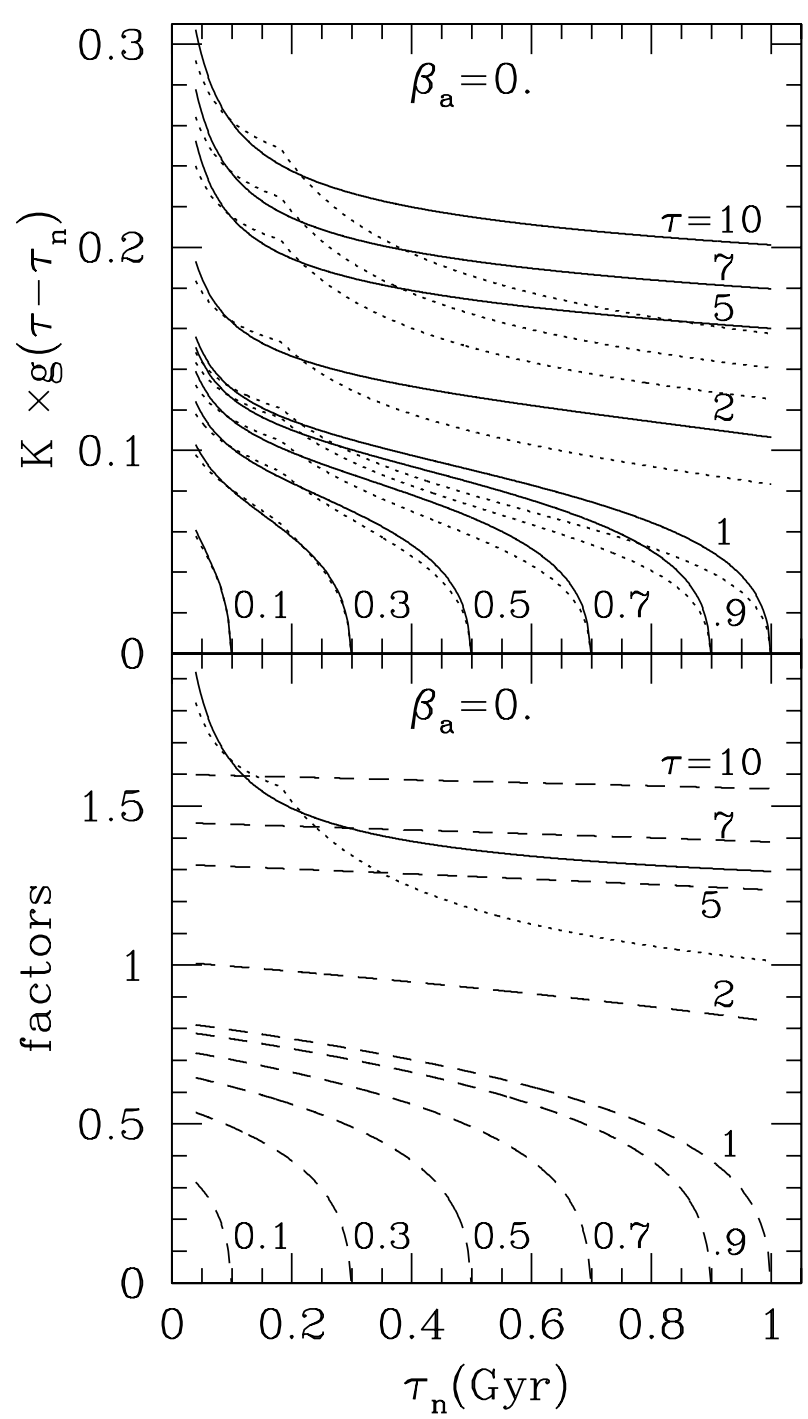

Fig. A.3. Lower panel: factors in the RHS of Eq. (A.10) for a flat distribution of $A$. The dashed lines show the $\tau$ dependent term and are labeled with the $\tau$ value in Gyr. Notice how, as $\tau$ increases toward $\tau_{\mathrm{n}, \mathrm{x}}=1 \mathrm{Gyr}$ the baseline on $\tau_{\mathrm{n}}$ increases, while for $\tau>1$ the curves just shift upward. Upper panel: resulting $g$ function in arbitrary units. Solid (dotted) lines result from using Eq. (A.10) with $f_{1}^{\mathrm{W}}\left(f_{2}^{\mathrm{W}}\right)$.

Inserting Eqs. (A.7) in (25), the $g\left(\tau, \tau_{\mathrm{n}}\right)$ function is derived as:

$g\left(\tau, \tau_{\mathrm{n}}\right) \propto \begin{cases}0 & \text { for } \tau \leq \tau_{1} \\ \left.f_{1,2}^{\mathrm{W}}\left[\left(\tau-\tau_{\mathrm{n}}\right)^{\tilde{\beta}_{\mathrm{a}}}-\tau_{\mathrm{gw}, \mathrm{i}}^{\tilde{\beta}_{\mathrm{a}}}\right)\right] & \text { for } \tau_{1} \leq \tau \leq \tau_{2}\end{cases}$

with $\tilde{\beta}_{\mathrm{a}}=0.25\left(1+\beta_{\mathrm{a}}\right), \tau_{1}=\tau_{\mathrm{n}}+\tau_{\mathrm{gw}, \mathrm{i}}, \tau_{2}=\tau_{\mathrm{n}}+\tau_{\mathrm{gw}, \mathrm{x}}$, and where the third branch has been neglected because it is assumed that the maximum gravitational delay is larger than the Hubble time for every $\tau_{\mathrm{n}}$.

The number of systems which have a nuclear delay $\tau_{\mathrm{n}}$ and a total delay up to $\tau$ is proportional to two factors: one $\left(f_{1,2}^{\mathrm{W}}\right)$ describing the systematics with $M_{\mathrm{DD}}$, the other scaling with the width of the parameter space in $\tau_{\mathrm{gw}}$. The dependence on the distribution of the separations appears in the exponents of both factors. The lower panel of Fig. A.3 illustrates these two factors for $\beta_{\mathrm{a}}=0$, which corresponds to a flat distribution of final separations. The $\tau$ dependent quantity ${ }^{11}$ (dashed lines) is larger when the total delay is larger: more systems merge within a longer total delay. At fixed $\tau$, this factor decreases for increasing $\tau_{\mathrm{n}}$, as the available range in $\tau_{\mathrm{gw}}$ decreases. At long total delay times this effect becomes less important, since the upper limit to $\tau_{\mathrm{n}}$ is of $1 \mathrm{Gyr}$ only. The solid and the dotted lines in the lower panel of Fig. A.3 show respectively the $f_{1}^{\mathrm{W}}$ and $f_{2}^{\mathrm{W}}$ factors, which account for the systematics of the gravitational delay with the nuclear delay: longer $\tau_{\mathrm{n}}$ correspond to lower $m_{2}$, and then to less massive $M_{\mathrm{DD}}$. At fixed $A$, systems with lower $M_{\mathrm{DD}}$ are diluted over a larger $\tau_{\mathrm{gw}}$ range: this effect is reflected on the decreasing $f_{1,2}^{\mathrm{W}}$ factors with increasing $\tau_{\mathrm{n}}$, and is more pronounced for $f_{2}^{\mathrm{W}}$ because of the additional systematics with the range $\left(M_{\mathrm{DD}, \mathrm{x}}-M_{\mathrm{DD}, \mathrm{n}}\right)$, which gets smaller as $\tau_{\mathrm{n}}$ increases (see Fig. A.2).

The upper panel in Fig. A.3 shows the resulting (nonnormalized ${ }^{12}$ ) function $g\left(\tau, \tau_{\mathrm{n}}\right)$ for the two considered distributions of $M_{\mathrm{DD}}$. By construction, the $g$ functions are zero for $\tau_{\mathrm{n}} \geq \tau-\tau_{\mathrm{gw}, \mathrm{i}}$ (see Eq. (A.10)). The fraction of systems within a given total delay time $\tau$ decreases as $\tau_{\mathrm{n}}$ increases, a dependence which is more pronounced when the variation in the range of $M_{\mathrm{DD}}$ with $m_{2}$ is accounted for (i.e. when using $f_{2}^{\mathrm{W}}$ ). At any $\tau_{\mathrm{n}}$ the fraction of systems with a delay time smaller than $\tau$ increases with $\tau$. The variation of $g$ with $\tau_{\mathrm{n}}$ and $\tau$ depends on the distribution function of the final separations $A$, as discussed in Sect. 4.3 and illustrated in Fig. 5.

\section{A.4. The differential distribution function of the delay times}

It its generic form, the Leibniz integral rule is:

$\frac{\mathrm{d}}{\mathrm{d} z} \int_{a(z)}^{b(z)} f(z, x) \mathrm{d} x=\int_{a(z)}^{b(z)} \frac{\partial f}{\partial z} \mathrm{~d} x+f(z, b) \frac{\mathrm{d} b}{\mathrm{~d} z}-f(z, a) \frac{\mathrm{d} a}{\mathrm{~d} z}$.

This can be applied to Eq. (32), with

$z=\tau \quad x=\tau_{\mathrm{n}} \quad f(z, x)=n\left(\tau_{\mathrm{n}}\right) \cdot g\left(\tau, \tau_{\mathrm{n}}\right)$

$a=\tau_{\mathrm{n}, \mathrm{i}} \quad b=\min \left(\tau_{\mathrm{x}}, \tau\right)$.

Let's consider in turn the three additive terms at the right hand side of the Leibniz rule. The last term is equal to:

$-\left[n\left(\tau_{\mathrm{n}}\right) g\left(\tau, \tau_{\mathrm{n}}\right)\right]_{\tau_{\mathrm{n}}=\tau_{\mathrm{n}, \mathrm{i}}} \frac{\mathrm{d} \tau_{\mathrm{n}, \mathrm{i}}}{\mathrm{d} \tau}=0$

because $\tau_{\mathrm{n}, \mathrm{i}}$ is constant.

The second term is equal to:

$\left[n\left(\tau_{\mathrm{n}}\right) g\left(\tau, \tau_{\mathrm{n}}\right)\right]_{\tau_{\mathrm{n}}=\min \left(\tau_{\mathrm{n}, \mathrm{x}}, \tau\right)} \frac{\mathrm{d} \min \left(\tau_{\mathrm{n}, \mathrm{x}}, \tau\right)}{\mathrm{d} \tau}=0$

because

(i) in $\tau \geq \tau_{\mathrm{n}, \mathrm{x}}: \frac{\mathrm{d} \tau_{\mathrm{n}, \mathrm{x}}}{\mathrm{d} \tau}=0$ since $\tau_{\mathrm{n}, \mathrm{x}}$ is constant;

11 A small value of $\tau_{\mathrm{gw}, \mathrm{i}}=0.001 \mathrm{Gyr}$ has been used in this figure.

12 A proper definition requires $g\left(\tau_{\mathrm{gw}, \mathrm{X}}+\tau_{\mathrm{n}}, \tau_{\mathrm{n}}\right)=1$ at every $\tau_{\mathrm{n}}$, which is a relation between $\tau_{\mathrm{gw}, \mathrm{x}}$ and $\tau_{\mathrm{n}}$. Since for the WIDE $D D$ scheme we consider $\tau<\tau_{\mathrm{gw}, \mathrm{x}}$ for every $\tau_{\mathrm{n}}$ the normalization factor is not important. 
(ii) in $\tau \leq \tau_{\mathrm{n}, \mathrm{x}}: g\left(\tau, \tau_{\mathrm{n}}=\tau\right)=0$ by construction, since the $g$ function is null in $\tau_{\mathrm{n}} \geq \tau-\tau_{\mathrm{gw}, \mathrm{i}}$ (see Eq. (25)).

Therefore, only the first term is left:

$f_{\mathrm{Ia}}^{\mathrm{DD}}(\tau)=\int_{\tau_{\mathrm{n}, \mathrm{i}}}^{\min \left(\tau_{\mathrm{n}, \mathrm{x}}, \tau\right)} n\left(\tau_{\mathrm{n}}\right) \frac{\partial g}{\partial \tau} \mathrm{d} \tau_{\mathrm{n}}$.

The $g\left(\tau, \tau_{\mathrm{n}}\right)$ function is continuous (see Eq. (25)) and thus its derivative can be computed in all its points. However, the function presents cusps in $\tau=\tau_{\mathrm{n}}+\tau_{\mathrm{gw}, \mathrm{i}}$ and in $\tau=\tau_{\mathrm{n}}+\tau_{\mathrm{gw}, \mathrm{x}}$, where the derivative will not be continuous.

Equation (A.10) (WIDE DDs) can be written as:

$g\left(\tau, \tau_{\mathrm{n}}\right) \propto \begin{cases}0 & \text { for } \tau_{\mathrm{n}} \geq \tau-\tau_{\mathrm{gw}, \mathrm{i}} \\ f_{1,2}^{\mathrm{W}}\left[\left(\tau-\tau_{\mathrm{n}}\right)^{0.25\left(1+\beta_{\mathrm{a}}\right)}-\tau_{\mathrm{gw}, \mathrm{i}}^{0.25\left(1+\beta_{\mathrm{a}}\right)}\right] & \text { for } \tau_{\mathrm{n}} \leq \tau-\tau_{\mathrm{gw}, \mathrm{i}}\end{cases}$ for delay times up to the Hubble time. Upon derivation this equation yields:

$\frac{\partial g}{\partial \tau} \propto \begin{cases}f_{1,2}^{\mathrm{W}}\left(\tau-\tau_{\mathrm{n}}\right)^{-0.75+0.25 \beta_{\mathrm{a}}} & \text { for } \tau_{\mathrm{n}} \leq \tau-\tau_{\mathrm{gw}, \mathrm{i}} \\ 0 & \text { for } \tau_{\mathrm{n}} \geq \tau-\tau_{\mathrm{gw}, \mathrm{i}}\end{cases}$

Inserting Eqs. (A.12) into (A.11) the distribution function of the delay times for the double degenerate systems in the WIDE DD evolutionary scheme is obtained as:

$f_{\mathrm{Ia}}^{\mathrm{DD}}(\tau) \propto \int_{\tau_{\mathrm{n}, \mathrm{i}}}^{\min \left(\tau_{\mathrm{n}, \mathrm{x}}, \tau\right)} n\left(\tau_{\mathrm{n}}\right) S^{\mathrm{W}}\left(\tau, \tau_{\mathrm{n}}\right) \mathrm{d} \tau_{\mathrm{n}}$

with

$S^{\mathrm{W}}\left(\tau, \tau_{\mathrm{n}}\right)= \begin{cases}f_{1,2}^{\mathrm{W}}\left(\tau-\tau_{\mathrm{n}}\right)^{-0.75+0.25 \beta_{\mathrm{a}}} & \text { for } \tau_{\mathrm{n}} \leq \tau-\tau_{\mathrm{gw}, \mathrm{i}} \\ 0 & \text { for } \tau_{\mathrm{n}} \geq \tau-\tau_{\mathrm{gw}, \mathrm{i}}\end{cases}$

For the CLOSE DD formulation, it's convenient to notice that the third branch in Eq. (31) requires $\tau \geq \tau_{\mathrm{n}}+\tau_{\mathrm{gw}, \mathrm{x}}\left(\tau_{\mathrm{n}}\right)$, and therefore exists only if the total delay time considered is sufficiently long: $\tau \geq \tau_{\mathrm{n}, \mathrm{i}}+\tau_{\mathrm{gw}, \mathrm{x}}\left(\tau_{\mathrm{n}, \mathrm{i}}\right)$. Therefore, I re-write Eq. (31) as:

- if $\tau \leq \tau_{\mathrm{n}, \mathrm{i}}+\tau_{\mathrm{gw}, \mathrm{x}}\left(\tau_{\mathrm{n}, \mathrm{i}}\right):$
$g\left(\tau, \tau_{\mathrm{n}}\right)= \begin{cases}\frac{\left(\tau-\tau_{\mathrm{n}}\right)^{1+\beta g}-\tau_{\mathrm{g}, \mathrm{i}}^{1+\beta \mathrm{g}}}{\tau_{\mathrm{gw}, \mathrm{i}}^{1+\beta g}-\tau_{\mathrm{gw}, \mathrm{i}}^{1+\beta g}} & \text { for } \tau_{\mathrm{n}} \leq \tau-\tau_{\mathrm{gw}, \mathrm{i}} \\ 0 & \text { for } \tau_{\mathrm{n}} \geq \tau-\tau_{\mathrm{gw}, \mathrm{i}}\end{cases}$

- if $\tau \geq \tau_{\mathrm{n}, \mathrm{i}}+\tau_{\mathrm{n}, \mathrm{x}}\left(\tau_{\mathrm{n}, \mathrm{i}}\right)$ :

$g\left(\tau, \tau_{\mathrm{n}}\right)= \begin{cases}1 & \text { for } \tau_{\mathrm{n}} \leq \tau_{\mathrm{n}}^{\star} \\ \frac{\left(\tau-\tau_{\mathrm{n}}\right)^{1+\beta g}-\tau_{\mathrm{gw}, \mathrm{i}}^{1+\beta g^{2}}}{\tau_{\mathrm{gw}, \mathrm{g}}-\tau_{\mathrm{gw}, \mathrm{i}}^{1+\beta g}} & \text { for } \tau_{\mathrm{n}}^{\star} \leq \tau_{\mathrm{n}} \leq \tau-\tau_{\mathrm{gw}, \mathrm{i}} \\ 0 & \text { for } \tau_{\mathrm{n}} \geq \tau-\tau_{\mathrm{gw}, \mathrm{i}}\end{cases}$ where $\tau_{\mathrm{n}}^{\star}$ is the solution of the equation $\tau=\tau_{\mathrm{n}}+\tau_{\mathrm{gw}, \mathrm{x}}\left(\tau_{\mathrm{n}}\right)$. The derivative is then:

- if $\tau \leq \tau_{\mathrm{n}, \mathrm{i}}+\tau_{\mathrm{gw}, \mathrm{x}}\left(\tau_{\mathrm{n}, \mathrm{i}}\right)$ :

$\frac{\partial g}{\partial \tau} \propto \begin{cases}\frac{\left(\tau-\tau_{\mathrm{n}} \beta_{\mathrm{g}}\right.}{\tau_{\mathrm{gw}, \mathrm{x}}^{1+\beta,}-\tau_{\mathrm{gw}, \mathrm{i}}^{1+\beta \mathrm{g}}} & \text { for } \tau_{\mathrm{n}} \leq \tau-\tau_{\mathrm{gw}, \mathrm{i}} \\ 0 & \text { for } \tau_{\mathrm{n}} \geq \tau-\tau_{\mathrm{gw}, \mathrm{i}}\end{cases}$

- if $\tau \geq \tau_{\mathrm{n}, \mathrm{i}}+\tau_{\mathrm{n}, \mathrm{x}}\left(\tau_{\mathrm{n}, \mathrm{i}}\right)$ :

$\frac{\partial g}{\partial \tau} \propto \begin{cases}0 & \text { for } \tau_{\mathrm{n}} \leq \tau_{\mathrm{n}}^{\star} \\ \frac{\left(\tau-\tau_{\mathrm{n}}\right)^{\beta g}}{\tau_{\mathrm{gw}, \mathrm{x}}^{1+\beta g}-\tau_{\mathrm{gw}, \mathrm{i}}^{1+\beta g}} & \text { for } \tau_{\mathrm{n}}^{\star} \leq \tau_{\mathrm{n}} \leq \tau-\tau_{\mathrm{gw}, \mathrm{i}} \\ 0 & \text { for } \tau_{\mathrm{n}} \geq \tau-\tau_{\mathrm{gw}, \mathrm{i}}\end{cases}$

The last two equations can be written in a compact form as:

$\frac{\partial g}{\partial \tau} \propto \begin{cases}f^{\mathrm{C}}\left(\tau, \tau_{\mathrm{n}}\right) & \text { for } \tau_{\mathrm{n}} \leq \tau-\tau_{\mathrm{gw}, \mathrm{i}} \\ 0 & \text { for } \tau_{\mathrm{n}} \geq \tau-\tau_{\mathrm{gw}, \mathrm{i}}\end{cases}$

with

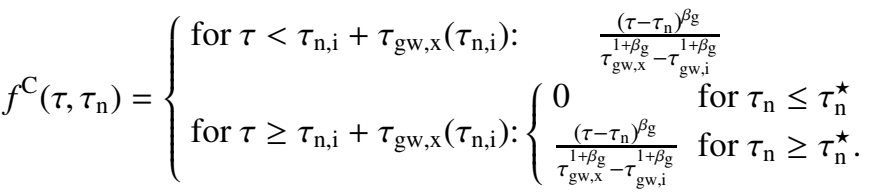

Inserting the last two equations into Eq. (A.11), and splitting the integration over $\tau_{\mathrm{n}}$ in two parts, one from $\tau_{\mathrm{n}, \mathrm{i}}$ to $\tau_{\mathrm{n}}^{\star}$ and the other from $\tau_{\mathrm{n}}^{\star}$ to the upper limit $\left(\min \left(\tau_{\mathrm{n}, \mathrm{x}}, \tau\right)\right)$, the distribution of the delay times is obtained as:

$f_{\mathrm{Ia}}^{\mathrm{DD}}(\tau)=\int_{\tau_{\mathrm{n}, \mathrm{nf}}}^{\min \left(\tau_{\mathrm{n}, \mathrm{x}}, \tau\right)} n\left(\tau_{\mathrm{n}}\right) S^{\mathrm{C}}\left(\tau, \tau_{\mathrm{n}}\right) \mathrm{d} \tau_{\mathrm{n}}$

with

$S^{\mathrm{C}}\left(\tau, \tau_{\mathrm{n}}\right)= \begin{cases}\frac{\left(\tau-\tau_{\mathrm{n}}\right)^{\beta_{\mathrm{g}}}}{\tau_{\mathrm{gw}, \mathrm{x}}^{1+\beta g}-\tau_{\mathrm{gw}, \mathrm{i}}^{1+\beta_{\mathrm{g}}}} & \text { for } \tau_{\mathrm{n}} \leq \tau-\tau_{\mathrm{gw}, \mathrm{i}} \\ 0 & \text { for } \tau_{\mathrm{n}} \geq \tau-\tau_{\mathrm{gw}, \mathrm{i}}\end{cases}$

and

$\tau_{\mathrm{n}, \mathrm{inf}}=\left\{\begin{array}{l}\tau_{\mathrm{n}, \mathrm{i}} \text { for } \tau<\tau_{\mathrm{n}, \mathrm{i}}+\tau_{\mathrm{gw}, \mathrm{x}}\left(\tau_{\mathrm{n}, \mathrm{i}}\right) \\ \tau_{\mathrm{n}}^{\star} \text { for } \tau \geq \tau_{\mathrm{n}, \mathrm{i}}+\tau_{\mathrm{gw}, \mathrm{x}}\left(\tau_{\mathrm{n}, \mathrm{i}}\right) .\end{array}\right.$ 